\title{
Molecular and cellular differentiation during the early shell field development in Lymnaea stagnalis
}

\author{
Dissertation \\ zur Erlangung des mathematisch-naturwissenschaftlichen Doktorgrades \\ "Doctor rerum naturalium" \\ der Georg-August-Universität Göttingen
}

im Promotionsprogramm Geowissenschaften / Geographie

der Georg August University School of Science (GAUSS)

vorgelegt von

Jennifer Hohagen

aus Wuppertal

Göttingen, 2013 


\section{Betreuungsausschuss}

Prof. Dr. Daniel J. Jackson, Courant Research Centre Geobiology, Fakultät für Geowissenschaften, Georg-August-Universität Göttingen, Germany

Prof. Dr. Joachim Reitner, Abteilung Geobiologie, Fakultät für Geowissenschaften, Georg-August-Universität Göttingen, Germany

\section{Mitglieder der Prüfungskommission}

Referent:

Prof. Dr. Daniel J. Jackson, Courant Research Centre Geobiology, Fakultät für Geowissenschaften, Georg-August-Universität Göttingen, Germany

Korreferent:

Prof. Dr. Joachim Reitner, Abteilung Geobiologie, Fakultät für Geowissenschaften, Georg-August-Universität Göttingen

Prof. Dr. Volker Thiel, Abteilung Geobiologie, Fakultät für Geowissenschaften, GeorgAugust-Universität Göttingen

Jun. Prof. Dr. Gregor Bucher, Abteilung Entwicklungsbiologie, Johann-FriedrichBlumenbach- Institut für Zoologie und Anthropologie, Georg-August-Universität Göttingen

Jun. Prof. Dr. Frank Melzner, Arbeitsgruppe Ökophysiologie; Helmholtzzentrum für Ozeanforschung Kiel

Dr. Frédéric Marin, BioME group, Laboratoire Biogéosciences, Universite de Bourgogne, Dijon, France.

Tag der mündlichen Prüfung: 16.12.2013 


\section{Versicherung}

Hiermit versichere ich an Eides statt, dass die Dissertation mit dem Titel "Molecular and cellular differentiation during the early shell field development in Lymnaea stagnalis" selbständig und ohne unerlaubte Hilfe angefertigt wurde.

Göttingen, den 18.11.2013 


\section{Contents}

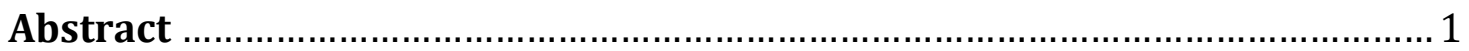

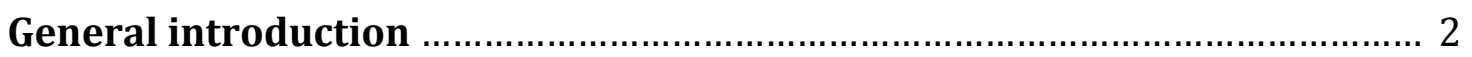

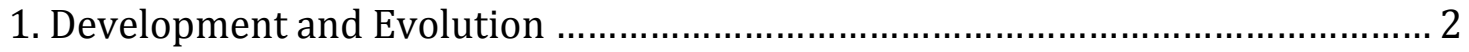

2. The evolutionary importance of the molluscan shell ......................................... 4

3. The molecular basis of evolutionary innovations .......................................... 9

\section{Chapter 2}

An ancient process in a modern mollusc: early development of the shell in Lymnaea stagnalis

\section{Chapter 3}

A whole mount in situ hybridisation protocol for the re-emerging molluscan model Lymnaea stagnalis

\section{Chapter 4}

Notch signalling during the early shell field development in Lymnaea stagnalis.

General discussion 


\begin{abstract}
Since the evolutionary emergence of the molluscan shell in the Pre-Cambrian, diverse shell forms have evolved, which are adapted to various ecological environments. The innovation of the shell facilitated the vast morphological and ecological diversification of the Mollusca, but its formation during the early embryogenesis is poorly understood. Many ontogenetic events associated with larval shell formation are evolutionarily conserved. Particularly, the initial specification of the responsible organ, the molluscan shell field, often coincides with a direct cell-cell contact between the prospective shell field cells and underlying endodermal cells. This observation raised the hypothesis of a contact-mediated induction event that specifies the molluscan shell field. Until now, this remains the canonical theory of molluscan shell field specification. In this thesis, I evaluate the role of an ancient intercellular signalling pathway - the Notch pathway - in the shell field specification of the derived gastropod Lymnaea stagnalis. The cellular arrangements and differentiation events during its early shell field developments are shown to be in agreement with a specification via contact-mediated induction. The spatial expression of core components of the Notch pathway does not support a deployment of Notch signalling in this process. Rather, Notch signalling seems to function during the early neurogenesis. Also, the pharmacological inhibition of Notch signalling during the presumably specifying contact event does not alter the shell field appearance, but prevents the correct cellular differentiation of the archenteron. This thesis represents the first set of investigations into the molecular regulators of shell field specification. Knowledge on the molecular basis of shell field development in L. stagnalis represents a first step towards further studies on a range of molluscan representatives. Such comparative studies will allow for inferences about the ancestral structure of the molecular framework that underlies shell development, and thus the genetic conditions under which the molluscan shell first arose.
\end{abstract}




\section{Chapter 1}

\section{General introduction}

\section{Development and evolution}

\subsection{The sudden rise of today's metazoan diversity}

Most extant animal groups diversified rapidly during a geologically brief period between $\sim 540$ and 500 million years ago. Multifarious theories have been invoked to explain this abrupt increase in both diversity and disparity, commonly referred to as the "Cambrian explosion". Proposed causes of the Cambrian explosion range from changing environmental conditions, ecological drivers like evolutionary arms races and the emergence of complex food webs to intrinsic alterations of genome architectures [1-4]. Nowadays, these mutually non-exclusive hypotheses have been integrated into an interpretation of the Cambrian explosion as an evolutionary cascade driven by interlinked abiotic, ecologic and genetic factors $[5,6]$.

In this scenario, pre-Cambrian genomic reorganisations generated the develop-

mental systems for building novel body plans and structures that characterise the extant animal crown groups [5, 7]. The evolution of these novel morphological features is thought to have enabled the later diversification during the early Cambrian $[3,6]$. Indeed, the first appearance of many animal crown groups and their characteristics predates their diversification during the Cambrian explosion for more than 100 million years $[5,6]$. Therefore, the evolution of developmental programs that allow for the acquisition of beneficial morphologies probably provided the background for later diversification in a permissive environment $[3,5,8]$.

\subsection{Morphological innovations as driving factor for evolutionary diversification}

Novel morphological features often evolve as defining synapomorphies at the base of a particular clade $[3,7]$. Such morphological innovations have been thought to facilitate evolutionary success by means of species diversity, the appearance of higher taxa and/or morphological disparity [9]. Commonly proposed mechanisms rest on an adaptive potential of morphological novelties; they might enable the occupation of new ecological niches and therefore reduce competition and promote specialisation and speciation (reviewed in [9]). Just few studies specifically test the 
concept of morphological innovations as trigger for evolutionary diversification [10]. This concept has been controversially discussed. Experimentally, it is difficult to approach how the emergence of a novel feature that characterises a lineage translates into a fitness increase of the individual that is subject to natural selection [9]. However, many clades establish their maximum morphological disparity shortly after the acquisition of their key apomorphies (independent from mass extinctions)[3]. Furthermore, many adaptive radiation events simply required certain structures to be in place, such as the invasion of the air by insects is bound to the evolution of their wings [8]. Evidently, both an increase in diversity and disparity is associated with the evolutionary emergence of novel morphological traits, and often these novel morphological features represent synapomorphies of the respective lineages.

\subsection{Morphological innovations reflect changes in development}

Morphological innovations can either originate from pre-existing structures or evolve de novo and can subsequently be adapted to a new purpose $[8,11]$. In both cases, the novel morphological feature originates in an individual organism and requires changes in its individual development. The evolutionary fixation of such ontogenetic changes could be perceived as reflecting the establishment of a new "developmental program". A developmental program represents a sequence of hierarchically organised events. These events are governed by the concerted action of distinct subsets of genes. Evolutionary alterations in their composition and spatial and temporal employment can result in new developmental programs allowing for the formation of novel morphological structures. Novel body structures lead to a new phenotype that natural selection will act upon and will determine whether that phenotype and hence its underlying novel genetic subset will persist. 


\section{The evolutionary importance of the molluscan shell}

2.1. The innovation of the shell was a major factor for the evolutionary success of the Mollusca

From an evolutionary perspective, molluscs are one of the most successful animal groups. They display among the highest diversity in body plans in any metazoan group, spread over around 200,000 extant species [12, 13]. Much of this evolutionary success can be attributed to the innovation of a structure with multifarious physiological and ecological benefits: the external shell. The shell is thought to function as solid support for the soft body of molluscs and is likely to bear adaptive potential for ecological diversification [14]. But most commonly, the ability to produce external calcified structures like the molluscan shell is interpreted as part of a widespread feedback loop between the invention of predatory and protective biomineralised structures during the Cambrian explosion [6]. Indeed, the fossils that might represent the most ancient molluscs are part of the mass skeletonisation event at the Precambrian-Cambrian boundary [15]. Earlier findings from the Ediacaran ( 635-541 MYA) have molluscan affinities, e.g. the famous Kimberella fossil, but do not reveal a biomineralised shell yet. By the Lower Cambrian $(\sim 541$ 513 MYA), a variety of shelled molluscan forms had already evolved. These early molluscan forms are part of the so-called "small shelly fossils", a diverse microscopic fossil assemblage, and are assigned to molluscs based on similarities in the shape and microstructure of their shells [15]. Among these shelled Cambrian fossils, three of today's major molluscan lineages are already represented (the externally shelled gastropods, bivalves and monoplacophorans), placing the origin and earliest radiation of the Mollusca before the Cambrian explosion [13]. Therefore, the innovation of an external calcified shell preceded the rapid morphological and ecological diversification of the Mollusca and was most likely a major driving factor [16]. Today's molluscan diversity is almost completely contained by one monophyletic group that is comprised of gastropods, bivalves, monoplacophorans, scaphopods and cephalopods: the Conchifera whose main synapomorphy is the external shell [17].

\subsection{Internal molluscan relationships and the origin of the shell}

Although the conchiferan molluscs are by far the most species-rich and ecologically dominant molluscan lineage, other extant molluscan groups exist that bear 
mineralised sclerites or shell plates but no rigid shells (Fig. 1). Polyplacophorans reveal eight dorsal shell plates whereas the only mineralised structures of the Neomeniomorpha (also known as Solenogastres) and Chaetodermomorpha (Caudofoveata) are numerous sclerites, which are embedded in the cuticle over nearly the entire body surface [17]. The shell-less, vermiform Neomeniomorpha and Chaetodermomorpha are commonly summarised as aplacophorans and are thought to be derived from polyplacophoran-like ancestors $[18,19]$. Recent phylogenetic studies placed the aplacophorans as monophyletic sister group of the polyplacophorans in the large clade Aculifera [16, 20,21] and confirmed the monophyly of the Conchifera $[16,20]$. Therefore, the deep molluscan phylogeny is currently inferred to reveal two major lineages: the shell-bearing Conchifera and the Aculifera, which are characterised by the presence of sclerites or shell plates (Fig. 1).

This relationship has implications for the origin and ancestral state of the molluscan shell. Either the mineralised epidermal structures of molluscs have evolved independently in the two lineages and are not homologous or they evolved progressively and today's shells and spicules depict different stages of this process [17].

In both cases, it is the innovation of the rigid, multi-layered shell as realised in the Conchifera that represents the event that preceded the rapid molluscan diversification during the Early Cambrian.

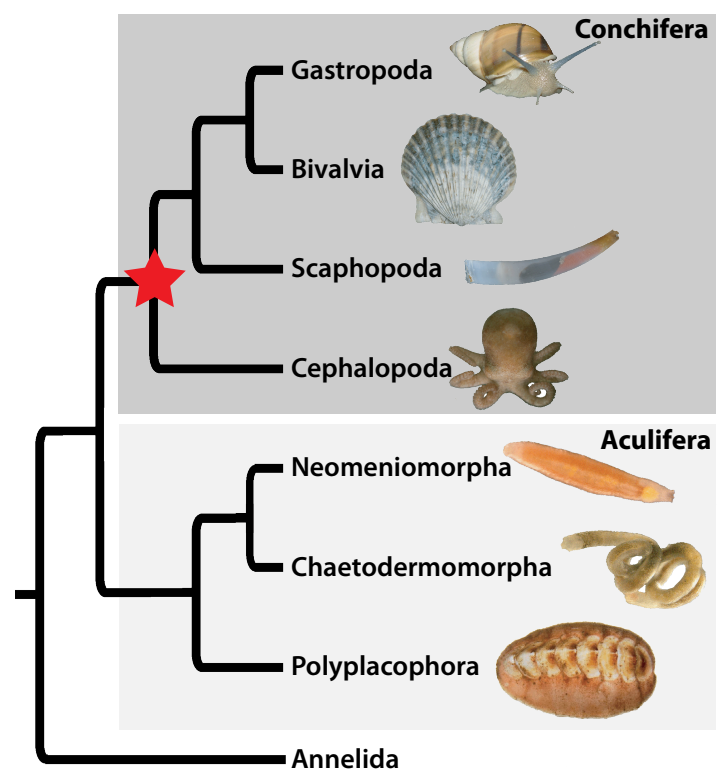

Fig.1. Deep molluscan relationships and the origin of the conchiferan shell. The shell evolved at the base of the Conchifera, one of the two major molluscan lineages (indicated by the asterisk). Adapted by permission from Macmillan Publishers Ltd: Nature [20], Copyright 2011. 


\subsection{The early shell-forming developmental program is conserved among molluscs}

Much of the evolutionary success of the conchiferan molluscs can be ascribed to the morphological variability of their shell. The various shapes, sizes, ornamentations and mineralogical textures of molluscan shells [22-24] reflect a functional plasticity that bears the potential of an adaptive radiation into new ecological territories.

In striking contrast to the morphological and functional diversity of the adult shell is the deep conservation of the developmental processes that initiate its construction. All conchiferan molluscs employ a homologous larval organ, the shell field, in the synthesis of the first shell. This larval shell field ultimately differentiates into the adult shell-forming organ, the mantle tissue that secretes the mature shell.

Molluscan shell field development involves several morphogenetic and cellular differentiation events that are evolutionarily conserved at least in all conchiferan classes (reviewed in [25]). The first conserved event concerns the initial differentiation of the future shell-forming cells. The cells of an ectodermal cell population assume a highly columnar shape, resembling a local thickening of the posttrochal dorsal region in gastropods, scaphopods and bivalves or the corresponding ectoblast in cephalopods. Later in development, the central part of the elongated ectoderm moves towards the underlying blastocoel in gastropods, scaphopods and bivalves to form the invaginated shell gland. In shelled cephalopods, the yolk mass underlies the thickened cell population, rendering an invagination impossible. Instead, the marginal cells bulge upwards and overgrow the central part to form a corresponding structure termed "shell sac". Therefore, the stage of an at least partial internalisation of the shell field epithel is another conserved feature of molluscan shell field development. Finally, an evagination of the shell gland and flattening of some formerly elongated cells later in development precede the first calcification throughout the Conchifera.

The widespread occurrence of these events in disparate molluscan classes implies the presence of a conserved developmental program for the initiation of shell construction. Such a developmental program would lie at the base of the Conchifera (Fig. 1) and represents a precondition for the evolutionary innovation of the molluscan shell. 


\subsection{The initial shell field development resembles a specification by induction}

Developmental programs for the formation of any morphological structure start with the determination of the embryonic region that will give rise to it: the specification of a progenitor field. Specified cells are determined to adopt a certain fate, which can be achieved by different mechanisms: autonomously (e.g. by inheritance of different cytoplasm contents after cell division) or by induction [26]. Cells that are specified by induction change their status in response to extracellular signals from other cells. Such signals can be transmitted by diffusible molecules (morphogens) or by direct cell-cell contacts.

The course of molluscan shell field development suggests an induction-dependent specification mechanism. The first visible cellular differentiation - the local thickening of the dorsal ectoderm - has been observed to coincide with an intimate contact of these elongated cells with endodermal cells of the underlying archenteron. Such a contact has been described in representatives of all conchiferan classes (reviewed in [25]) and has led to the idea that this contact is required for the specification of the future shell field cells and reflects a contact mediated induction event [27]. Until now, the hypothesis of a contact-dependent induction mechanism remains the most comprehensive theory of shell field specification in molluscs.

\subsection{Lymnaea stagnalis as re-emerging model for shell field development}

Most modern studies on molluscan development have been focused on the formation of other organ systems like the nervous and muscle systems, e.g. [28-30] and the larval prototroch [31] than on the early shell formation. The few studies on embryonic shell development do not describe the cellular arrangements and morphogenetic movements that predate the visible shell field differentiation. Instead, these previous cytological studies rather focused on the process of first shell secretion and associated cellular conditions than on the initial specification of the shell field (e.g. [32-36]). To date, there is no molluscan model system for investigating the early embryonic shell field development available.

The pulmonate gastropod Lymnaea stagnalis (Linnaeus, 1858) was once much used as a model for both molluscan development in general [37] and shell field development in particular [32,33]. More recently, L. stagnalis is re-employed as a model system for studies on the establishment of shell chirality [38, 39]. The shell field development of L. stagnalis reveals many of the conserved features described in

other conchiferan species [33]. In particular, the widespread contact between the 
early differentiating shell field cells and the underlying endodermal cells is present (Fig. 2). This allows for explicit testing of the theory of a contact-dependent induction, rendering L. stagnalis a suitable model for investigations into the mechanisms that mediate shell field specification.
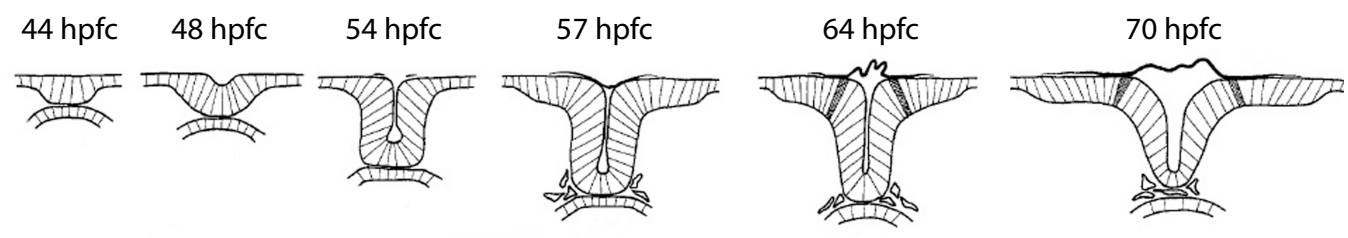

Fig. 2. The early shell field development in L. stagnalis. The first thickening and the later invagination of the shell field coincide with an intimate contact with underlying endodermal cells. Ages are indicated in hours post first cleavage (hpfc). Adapted by permission from Springer Science and Business Media from [33], Copyright 1977.

Such analyses require the previous determination of both the exact timing of critical events (contact establishment and first morphological differentiation) and the associated cellular conditions and tissue arrangements. In chapter 2, I provide a detailed temporal description of the early shell field development of L. stagnalis using modern techniques such as confocal laser microscopy.

This study represents the basis for identifying the molecular mechanisms that control shell field development in L. stagnalis. Future comparisons of this derived gastropod with early branching gastropods, representatives of the other conchiferan classes and ultimately non-shelled aculiferan species bear the potential to shed light on the molecular framework that underlies the evolution of the molluscan shell. 


\section{The molecular basis of evolutionary innovations}

\subsection{Evolutionary innovation is based on new use of old genes}

The emergence of a novel morphological structure like the molluscan shell is preceded by the evolution of a new developmental program that underlies its formation. Such a program can be perceived as a sequence of developmental events controlled by distinct subsets of genes. Any of these subsets evolves by itself using few general mechanisms. Gene duplication results in the existence of paralogues whose redundancy allows for the gain of a new function in one copy (neofunctionalisation). Changes in the spatial or temporal regulation of genes (heterotopy and heterochrony respectively) can place these genes into new developmental contexts, enable them to adopt new functions (pleiotropy) and can lead to novel interactions and the assembly of a new genetic subset (rewiring or "tinkering") [8]. Neofunctionalisation, heterotopy/heterochrony, pleiotropy and the rewiring of regulatory interactions are known as the main genetic mechanisms that underlie the emergence of novel morphological traits like the molluscan shell.

The common theme of these mechanisms is that they rest on the deployment of preexisting genetic elements in a new context rather than on the use of new genes. The role of de novo originated genes in the generation of evolutionary novel structures is far less understood [40]. Novel protein-coding genes arise from previously noncoding DNA sequence and encode products that do not share similarity with known proteins. Most of these novel genes are poorly characterised at the functional level, although there is growing evidence that the de novo emergence of protein-coding genes is more common than long-thought ([41-44]; reviewed in [40]). In molluscs, novel sequences are abundant in the adult shell-secreting mantle tissue and among the proteins occluded in the shell [45-49]. These lineage-specific genes and proteins are involved in the shell construction process and likely to participate in generating the morphological diversity of the adult molluscan shell. However, the developmental processes by which molluscan embryos initiate shell formation are deeply conserved and thought to have evolved at the base of the shelled molluscs (Fig. 1). The genes that control such an ancient developmental program are expected to be similarly conserved. 


\subsection{Morphological change evolves by alterations of gene regulatory networks}

Most of the protein-coding parts of metazoan genomes are highly conserved and cannot alone explain the emergence of clade-specific morphological features like the molluscan shell. Instead, evolutionary changes in animal morphology can often be attributed to alterations in the expression of genes that encode structurally conserved proteins. These proteins include transcription factors that regulate gene expression and signalling molecules that mediate the communication between cells and have been referred to as "toolkit proteins" [50]. Importantly, the number of toolkit proteins and the signalling pathways they form is limited; most by now studied developmental processes are controlled by a few dozen transcription factors and seven evolutionarily conserved signalling pathways which are shared by disparate and long-diverged taxa [8].

Toolkit proteins control developmental processes ranging from the establishment of broad territories in the early embryo to the fine-differentiation of morphological features displayed by the adult organism. The correct outcome of any developmental process relies on their correct spatial and temporal presence and abundance. The set of available regulatory proteins represents the "regulatory state" of a spatial domain, e.g. any cell, tissue or larger compartment [51]. Multiple interactions between the genes that encode regulatory proteins exist: regulatory genes can activate, repress, enhance and attenuate their own and each other's expression. These regulatory genes form together with the genomic sequences that control their expression (cis-regulatory elements) and their target genes complex gene regulatory networks. Thus, a gene regulatory network is composed of coding and non-coding genomic elements [51]. Any change in the corresponding genomic DNA sequences will affect the interactions within the gene regulatory network and alter the regulatory state of the cell or tissue. This will influence the developmental process this cell or tissue is involved in and ultimately lead to a change in morphology.

\subsection{The hierarchical architecture of gene regulatory networks}

Developmental gene regulatory networks represent the genetic foundation of developmental programs. The individual components of gene regulatory networks are arranged in distinct communicating subsets or sub-circuits (Fig. 3, p. 15). Different sub-circuits control different phases of the developmental process, i.e. specific sequential events of the developmental program. This results in a hierarchical 
architecture of the whole gene regulatory network $[51,52]$. The development of any morphological structure is initiated with the establishment of a progenitor field (specification), which is determined by the action of evolutionarily conserved subcircuits called kernels [51] or character identity networks [52]. The kernels interact with sub-circuits that define the spatial subdivision or patterning of the progenitor field. These sub-circuits reflect the regulatory state of the specified developing structure and control the activity of differentiation genes. Genes of the regulatory state sub-circuits encode transcription factors that drive the expression of downstream differentiation genes by binding to their cis-regulatory elements. The products of these differentiation genes exert the morphogenetic and cellular differentiation steps that complete an organogenetic process.

The interactions between the hierarchical levels of a gene regulatory network are not strictly linear; positive and negative feedback mechanisms are commonly employed both within and among the different levels (Fig. 3, arrows). Furthermore, gene regulatory networks are influenced by cis-regulatory genes encoding transcription factors that enhance or prevent the activity of a particular sub-circuit. These so called "Input/Output switches" are not implemented in the developmental gene regulatory network and can act on all levels of the gene regulatory network [51]. Genes encoding Input/Output switches are often expressed in response to intercellular signalling pathways. The genes that encode the signalling molecules have been referred to as "plug-ins" [51].

\subsection{Known elements of the early molluscan shell-forming gene regulatory network}

While the molecular basis of molluscan shell formation in the mature organism has begun to be elucidated $[45-47,49,53]$ the gene regulatory network that underlies the initiation of shell formation during embryogenesis remains elusive. So far, a limited number of genes associated with larval shell formation have been identified. The best-studied example represents the homeobox gene engrailed whose expression pattern during the early embryonic and larval development has been studied in disparate molluscan taxa [54-62]. The transcription factor engrailed is expressed in the larval shell field of gastropods, bivalves, scaphopods, cephalopods and polyplacophorans, which suggests a conserved function for engrailed during early molluscan shell development. In these taxa, engrailed expression is located in the shell-secreting cells at the shell field margin. Additionally, for the gastropods Ilyanassa obsoleta [54] and Lymnaea stagnalis [59] and the cephalopod Sepia 
officinalis [58] expression in the shell field or shell sac as soon as these structures are morphologically discernable or even earlier in the scaphopod Antalis entalis [56] has been reported.

The conserved spatial expression of engrailed in the shell field periphery has initially raised the hypothesis that engrailed is required for the process of shell formation (skeletogenesis) by demarcating biomineralising tissues [55] or maintaining the secretion of shell matrix proteins [59]. A skeletogenic function of engrailed is supported by its expression adjacent to cells concerned with shell and spicule formation in the polyplacophoran Lepidochitona caverna and the bivalve Transennella tantilla [55] and in cells surrounding artificially induced internal shell mass in I. obsoleta [54]. However, engrailed expression is transient and predates the first shell secretion in the cephalopod $S$. officinalis and is also not maintained in tissues forming the adult shell in the scaphopod A. entalis [56]. This renders an exclusively skeletogenic function of engrailed unlikely.

Alternatively, engrailed has been proposed to serve a more general function in the establishment of a compartment boundary between the developing shell field and the surrounding tissue [57]. This hypothesis is based on the co-expression of engrailed and another gene, decapentaplegic (dpp), in the gastropod Patella vulgata. Expression of engrailed and $d p p$ is found in adjacent but non-overlapping domains framing the transition between embryonic shell field and encompassing ectoderm [57]. The establishment of boundaries between embryonic compartments is a wellstudied function of engrailed during metazoan development and is also known to be exerted in cooperation with $d p p$, e.g. during boundary formation in the wing imaginal disc of Drosophila (reviewed in [63]).

Dpp is a member of the transforming growth factor- $\beta$ family of secreted signalling proteins. Aside from $P$. vulgata, the expression of $d p p$ during embryonic shell formation has been investigated in three other gastropod species (L. stagnalis, Haliotis asinina and Nipponacmea fuscoviridis] and the bivalve Saccostrea kegaki [38, 59, 60, $62,64,65]$. The expression of $d p p$ is not conserved among these species and reveals striking differences in terms of its location within the shell field and the spatial relation to engrailed-expressing cells.

In the limpets $P$. vulgata and $N$. fuscoviridis, dpp-expressing cells encircle the engrailed-expressing peripheral shell field cells [57, 62]. Similarly, dpp expression in the vetigastropod $H$. asinina assumes the shape of an incomplete ring around the shell field [64]. In the bivalve S. kegaki, dpp expression is located in two single cells 
directly above and below the peripheral, engrailed-expressing cells of the invaginating shell field. Later $d p p$ expression is restricted to the future hinge region of the expanding shell field [60], which is situated inside the expression domain of engrailed. In the pulmonate gastropod L. stagnalis, $d p p$ is expressed in the invaginated shell gland and later in the forming mantle in a bilaterally asymmetrical fashion [38, 59, 65]. Expression of $d p p$ in the future shell-forming area that predates the morphological differentiation of the shell field has exclusively been reported in H. asinina [64].

As a consequence of the lack of conservation in the expression patterns of $d p p$, individual hypotheses about possible functions of Dpp during molluscan shell formation have been formulated for each scenario. For S. kegaki, an early role for Dpp in restricting the expansion of shell field length along the dorsal midline to generate the bivalve-specific dumbbell-like shape and a later role in the hinge formation have been suggested [60]. For L. stagnalis and N. fuscoviridis, Dpp signalling has been proposed to control cell proliferation in the developing mantle tissue $[62,65]$. According to this hypothesis, asymmetric $d p p$ expression causes a morphogen gradient that induces asymmetric mantle expansion, which ultimately determines the coiled shape of the forming shell $[38,59,65]$.

An engagement in the shell coiling process of gastropods has also been shown for another member of the transforming growth factor- $\beta$ family, nodal, and its downstream target gene pitx in Lottia gigantea and Biomphalaria glabrata [66] and $N$. fuscoviridis [67]. In these species the asymmetrical expression of nodal and pitx near the developing shell field correlates with the body handedness including the direction of shell coiling but is known not to be the earliest symmetry-breaking event. Recently, a maternally inherited genomic locus has been identified that determines the body handedness in L. stagnalis [39]. Since shell coiling is a direct consequence of the establishment of body handedness, nodal and its upstream gene exert a mere indirect influence on shell field development.

Further known components of the molecular circuitry that underlies early molluscan shell formation include members of the Hox gene family. These transcription factors are well known for their role in patterning the anterior-posterior axis throughout the Metazoa and are known to take part in the shell field morphogeneses of the gastropods H. asinina, Gibbula varia and N. fuscoviridis [62, 64, 68, 69]. Most notably, Hox1 is continuously expressed in shell-secreting cells firstly of the expanding shell field and forming mantle edge and later in the anterior mantle 
margin in all investigated species. Similarly, the posterior Hox genes Post1 and Post2 are stably expressed during shell field morphogenesis in Gibbula and reveal fully or partially overlapping expression domains with Hox1. A role in the regulation of both the larval and adult shell secretion has been suggested for these constitutively expressed genes [69]. In contrast, Hox4 expression is initially detected after larval shell development is completed in the mantle tissue of both H. asinina and G. varia and has therefore been associated with the patterning process of the adult shell [68, 69]. The expression of Hox genes prior to the establishment of the morphologically differentiated shell field has not been observed.

Finally, nine genes have been identified that reveal dynamic spatial and temporal expression profiles in shell forming cells and tissues during the development of H. asinina [70]. Developmental changes in the expression of these genes can be linked to changes in shell properties like structure, colour or pattern. Five of these genes are evolutionarily conserved genes most of which exert general cellular functions such as protein folding or cell cycle regulation. The remaining four genes do not share similarity to any characterised protein and represent novel genes whose products are predicted to be secreted and to directly contribute to the construction of the shell.

3.6. The putative positions of genes associated with early shell formation within the network

The number of molluscan taxa in which the transcription factors and signalling molecules with evolutionarily conserved expression in shell forming tissues have been studied is increasing. However, none of these genes is likely to trigger the initiation of shell field development. Rather, engrailed, dpp and the Hox genes seem to function in the regional patterning and differentiation of a pre-defined shell field area. In terms of gene regulatory network terminology they take part in the regulatory state sub-circuits (Fig. 3). Also, at least $d p p$ and the Hox genes are expressed well after the initial shell field specification when the shell field is already morphologically differentiating. The specification is yet required to precede any visible differentiation. For the scaphopod A. entalis, early expression of engrailed in the prospective shell field area has been reported [56]. However, genes that specify the progenitor field of a homologues structure like the molluscan shell field would be expected to reveal highly conserved expression over the entire evolutionary 
distance [52]. Therefore, the genetic subset that specifies the future shell field cells (the most upstream "kernel" or "character identity network") is yet to be identified.

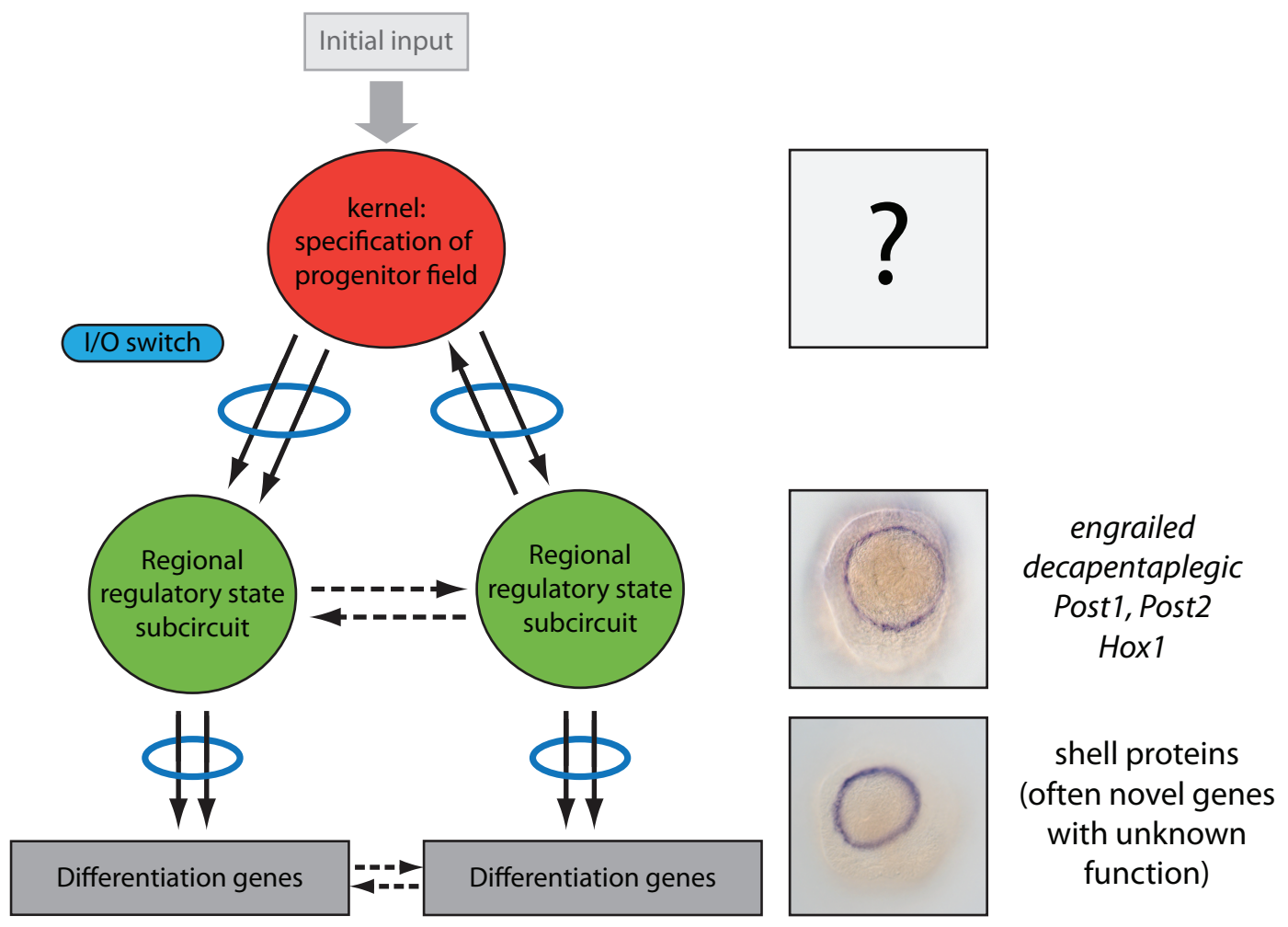

Fig. 3. The hierachical architecture of developmental gene regulatory networks. As any developmental gene regulatory network (GRN), the molluscan shell forming GRN is hierarchically organised. The genetic sub-circuit that specifies the molluscan shell is yet to be discovered. Depicted is the expression of engrailed (upper panel) and the novel shell protein coding gene contig 2185 (bottom panel, property of Ines Herlitze) in the larval shell field of L. stagnalis. Adapted by permission from Macmillan Publishers Ltd: Nature Reviews Genetics [51], Copyright 2009.

\subsection{A role for Notch signalling in molluscan shell field specification}

The most upstream component of the gene regulatory network underlying molluscan shell field development - the genes that specify the prospective shell field cells or kernel - can be predicted to reveal distinct characteristics. Firstly, these genes must have already been in place at the time when the molluscan shell originated more than 540 MYA. Secondly, their expression in the future shell field cells should be evolutionarily conserved within the Mollusca. Thirdly, they must be capable to interact with the presumably downstream regulatory genes engrailed, dpp, Hox1, Post 1 and Post2. And finally, if the molluscan shell field is truly specified by a 
contact-dependent induction process (p. 8), it should be possible to link their mode of operation with the contact event observed during shell field morphogenesis.

The Notch signalling pathway is an evolutionarily conserved signalling cascade present in all multicellular animals from sponges [71] to humans (reviewed in [72]) that meets most of the above predictions. Notch signalling is deployed repeatedly during the development of all metazoans and results in diverse outcomes depending on the developmental context [72]. In particular, Notch is one of only seven intercellular signalling pathways that together account for the vast majority of early cell fate decisions [73] and is known to communicate with numerous conserved pathways [72][74]. The core components of Notch signalling consist of a transmembrane ligand in one cell and a transmembrane receptor and a transcription factor in an adjacent cell. Active signalling is initiated by binding of the ligand to the receptor and leads to the transcriptional activation of downstream genes in the signal-receiving cell [75]. Hence, it acts via direct cell-cell contact, rather than by the release and diffusion of morphogens.

Preliminary data on Notch signalling during the development of the gastropod $H$. asinina (Jackson, unpublished data) has revealed persisting expression of the Notch ligand delta from gastrulation to the formation of a trochophore larva (Fig. 4). The expression of delta is sustained in the dorsal ectoderm before and after the shell field forms, rendering Notch signalling a likely candidate pathway to specify the molluscan shell field.
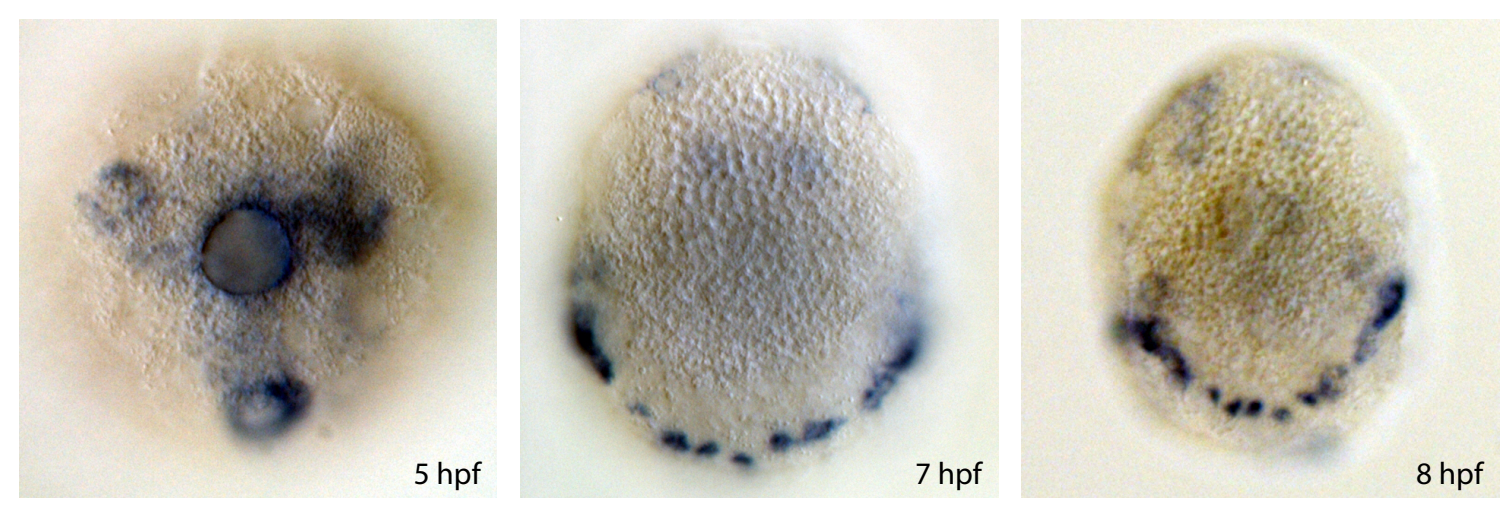

Fig. 4. Early developmental expression of the notch ligand delta in $\boldsymbol{H}$. asinina. Ages are indicated in hours post fertilisation (hpf). All embryos are shown from the dorsal side. Property of Daniel J. Jackson. 
3.8. Investigations into gene regulatory networks start with developmental gene expression data

A powerful strategy to plumb the underlying molecular framework of the development of a certain structure is analysing the temporal and spatial expression of genes engaged in its formation. Knowledge of the spatial distribution of gene transcripts at a particular time point can be used to infer their interactions and functions [76, 77]. A commonly employed technique for the study of spatial gene expression in a given tissue or structure is in situ hybridisation, often implemented on developmental stages as whole mount in situ hybridisation (WMISH). However, this technique is challenging if applied to a non-model organism like L. stagnalis. Experimental protocols for WMISH need to account for the unique characteristics displayed by any organism such as tissue architecture and composition and species-specific anatomical features. In order to achieve an optimal signal with minimal background the experimental procedure needs to provide sufficient access of the riboprobe to its target without overly compromising the morphological integrity. These are often contrasting requirements. The tolerance of any given whole mount for permeabilising procedures depends on various factors such as size, shape, internal architecture and cellular and molecular tissue composition. WMISH experiments on embryos can be further challenged by dynamic changes of these factors during ontogenesis. Thus, the experimental procedure of WMISH often needs to be optimised for different developmental stages. I therefore systematically compared the effects of key modifications to develop an optimised WMISH protocol for L. stagnalis that enables the investigation of the gene regulatory network that governs molluscan shell field specification. This study is presented in chapter 3.

Another approach to study gene expression within the developing embryo is quantitative real time PCR. This technique provides information about the timing and abundance of a gene's expression. Quantitative real time PCR (qRT-PCR) is a powerful tool to detect even subtle differences in gene expression in different tissues or developmental stages. Key to the reliability of qRT-PCR results is the normalisation of the resulting data. This is commonly done by comparison with an endogenous standard, usually by simultaneous amplification of internal reference genes. The choice of any internal reference genes is critical since the accuracy of expression data derived from qRT-PCR experiments relies on the constant expression level of the reference gene in all compared samples. Therefore, expression profiling based on internal reference genes requires the prior validation of their 
uniform expression [78]. I tested a number of candidate reference genes with putatively constant expression levels in several adult tissues and developmental stages of L. stagnalis to establish a validated set of reference genes for gene expression studies in L. stagnalis. Preliminary results of this project are summarised in appendix 1.

\subsection{Experimental approaches to investigate Notch signalling during shell field} specification

With the detailed spatial and temporal description of the shell field morphogenesis and an optimised experimental procedure for assessing developmental gene expression, the required resources are at hand that allow to assess the possible role of Notch signalling in shell field development.

If Notch signalling functions in mediating a contact-dependent "specification by induction" process, Notch pathway members are expected to be expressed in the future shell field. In particular, the prospective shell field cells and the endodermal cells of the underlying archenteron should each express at least one Notch ligand and receptor shortly before or during the contact establishment. I therefore performed WMISH experiments against presumably all Notch receptors and ligands on this critical stage.

A fundamentally different approach to prove the potential involvement of a particular molecular pathway is the experimental manipulation of this pathway and the interpretation of the resulting phenotypes. Disturbing the functioning of a candidate pathway during a particular process might also allow one to distinguish between a causal relationship or a mere coincidence of candidate gene expression and developmental event. For the Notch pathway, a pharmacological inhibitor (DAPT) is available that prevents the signal transduction. I applied this inhibitor to embryos of L. stagnalis during cleavage, gastrulation and formation of the trochophore larva, which comprises the shell field stages of the contact establishment and the first morphological differentiation.

This work on the developmental expression and the experimental inhibition of the Notch pathway in L. stagnalis is presented in chapter 4. 


\section{References}

1. Erwin DH: Disparity: morphological pattern and developmental context. Palaeontology 2007, 50:57-73.

2. Couso J: Segmentation, metamerism and the Cambrian explosion. Int J Dev Biol 2009, 53:1305-1316.

3. Hughes $\mathrm{M}$, Gerber $\mathrm{S}$, Wills M: Clades reach highest morphological disparity early in their evolution. Proc Natl Acad Sci U S A 2013, 110:13875-13879.

4. Sperling E, Frieder C, Raman A, Girguis P, Levin L, Knoll A: Oxygen, ecology, and the Cambrian radiation of animals. Proc Natl Acad Sci U S A 2013, 110:13446-13451.

5. Erwin D, Laflamme M, Tweedt S, Sperling E, Pisani D, Peterson K: The Cambrian Conundrum: Early Divergence and Later Ecological Success in the Early History of Animals. Science 2011, 334:1091-1097.

6. Smith M, Harper D: Causes of the Cambrian Explosion. Science 2013, 341:13551356.

7. Erwin DH: Early introduction of major morphological innovations. Acta Palaeontol Pol 1994, 38:281-294.

8. Carroll SB, Grenier JK, Weatherbee SD: From DNA to Diversity: Molecular Genetics and the Evolution of Animal Design. Oxford; Malden, Mass.: Blackwell Science; 2001.

9. Hunter JP: Key innovations and the ecology of macroevolution. Trends Ecol Evol 1998, 13:31-36.

10. Dumont E, Davalos L, Goldberg A, Santana S, Rex K, Voigt C: Morphological innovation, diversification and invasion of a new adaptive zone. Proc $R$ Soc $B$ 2012, 279:1797-1805.

11. Müller G, Wagner G: Novelty in evolution: restructuring the concept. Anпи Rev Ecol Syst 1991, 22:229-256.

12. Wanninger A, Koop D, Moshel-Lynch S, Degnan BM: Molluscan Evolutionary Development. In Phylogeny and Evolution of the Mollusca. Edited by Ponder WF, Lindberg DR. London: University of California Press, Ltd.; 2008:427-445.

13. Haszprunar G, Schander C, Halanych KM: Relationships of Higher Molluscan Taxa. In Phylogeny and Evolution of the Mollusca. Edited by Ponder WF, Lindberg DR. London: University of California Press, Ltd.; 2008:19-32.

14. Marin F, Luquet G: Molluscan shell proteins. Comp Ren Palevol 2004, 3:469-492.

15. Parkhaev PY: The Early Cambrian Radiation of Mollusca. In Phylogeny and 
Evolution of the Mollusca. Edited by Ponder WF, Lindberg DR. London: University of California Press, Ltd.; 2008:33-69.

16. Smith S, Wilson N, Goetz F, Feehery C, Andrade S, Rouse G, Giribet G, Dunn C: Resolving the evolutionary relationships of molluscs with phylogenomic tools. Nature 2011, 480:364-367.

17. Scheltema $\mathrm{AH}$, Schander $\mathrm{C}$ : Exoskeletons: tracing molluscan evolution. Venus 2006, 65:19-26.

18. Sutton MD, Briggs DE, Siveter DJ, Siveter DJ, Sigwart JD: A Silurian armoured aplacophoran and implications for molluscan phylogeny. Nature 2012, 490:9497.

19. Scherholz M, Redl E, Wollesen T, Todt C, Wanninger A: Aplacophoran Mollusks Evolved from Ancestors with Polyplacophoran-like Features. Curr Biol 2013,

20. Kocot KM, Cannon JT, Todt C, Citarella MR, Kohn AB, Meyer A, Santos SR, Schander C, Moroz LL, Lieb B, Halanych KM: Phylogenomics reveals deep molluscan relationships. Nature 2011, 477:452-456.

21. Vinther J, Sperling E, Briggs D, Peterson K: A molecular palaeobiological hypothesis for the origin of aplacophoran molluscs and their derivation from chiton-like ancestors. Proc $R$ Soc B 2012, 279:1259-1268.

22. Lowenstam HA, Weiner S: On biomineralization. New York: Oxford University Press, Inc.; 1989.

23. Abbott RT, Dance SP: Compendium of Seashells: a Full-color Guide to More Than 4,200 of the World's Marine Shells. El Cajon: Odyssey Publishing; 1998.

24. Meinhardt H: The Algorithmic Beauty of Sea Shells. Berlin Heidelberg: Springer; 2009.

25. Kniprath E: Ontogeny of the Molluscan Shell Field: a Review. Zool Script 1981, 10:61-79.

26. Salazar-Ciudad I: Morphological evolution and embryonic developmental diversity in metazoa. Development 2010, 137:531-539.

27. Raven CP: Morphogenesis in Limnaea stagnalis and its disturbance by lithium. J Exp Zool 1952, 121:1-77.

28. Dickinson A, Croll R: Development of the larval nervous system of the gastropod Ilyanassa obsoleta. J Comp Neurol 2003, 466:197-218.

29. Croll RP: Development of embryonic and larval cells containing serotonin, catecholamines, and FMRFamide-related peptides in the gastropod mollusc Phestilla sibogae. Biol Bull 2006, 211:232-247.

30. Hejnol A, Martindale MQ, Henry JQ: High-resolution fate map of the snail 
Crepidula fornicata: The origins of ciliary bands, nervous system, and muscular elements. Dev Biol 2007, 305:63-76.

31. Henry JQ, Okusu A, Martindale MQ: The cell lineage of the polyplacophoran, Chaetopleura apiculata: variation in the spiralian program and implications for molluscan evolution. Dev Biol 2004, 272:145-160.

32. Timmermans LPM: Studies on shell formation in molluscs. Neth J Zool 1969, 19:63-78.

33. Kniprath E: Zur Ontogenese des Schalenfeldes von Lymnaea stagnalis. Dev Gen Evol 1977, 181:11-30.

34. Kniprath E: Larval development of the shell and the shell gland in Mytilus (Bivalvia). Dev Gen Evol 1980, 188:201-204.

35. Eyster LS: Ultrastructure of early embryonic shell formation in the opisthobranch gastropod Aeolidia papillosa. Biol Bull 1983, 165:394-408.

36. Eyster L, Morse M: Early shell formation during molluscan embryogenesis, with new studies on the surf clam, Spisula solidissima. Am Zool 1984, 24:871882.

37. Raven CP: The Development of the Egg of Limnaea Stagnalis L. From the First Cleavage Till the Trochophore Stage, With Special Reference To Its" Chemical Embryology". Arch Neer Zool 1946, 7:353-434.

38. Shimizu K, Iijima M, Setiamarga D, Sarashina I, Kudoh T, Asami T, Gittenberger E, Endo K: Left-right asymmetric expression of dpp in the mantle of gastropods correlates with asymmetric shell coiling. EvoDevo 2013, 4:15.

39. Liu MM, Davey JW, Banerjee R, Han J, Yang F, Aboobaker A, Blaxter ML, Davison A: Fine Mapping of the Pond Snail Left-Right Asymmetry (Chirality) Locus Using RAD-Seq and Fibre-FISH. PLoS ONE 2013, 8:e71067.

40. Kaessmann $\mathrm{H}$ : Origins, evolution, and phenotypic impact of new genes. Genome Res 2010, 20:1313-1326.

41. Levine MT, Jones CD, Kern AD, Lindfors HA, Begun DJ: Novel genes derived from noncoding DNA in Drosophila melanogaster are frequently $\mathrm{X}$-linked and exhibit testis-biased expression. Proc Natl Acad Sci U S A 2006, 103:9935-9939.

42. Cai J, Zhao R, Jiang H, Wang W: De Novo Origination of a New Protein-Coding Gene in Saccharomyces cerevisiae. Genetics 2008, 179:487-496.

43. Knowles DG, McLysaght A: Recent de novo origin of human protein-coding genes. Genome Res 2009, 19:1752-1759.

44. Markljung E, Jiang L, Jaffe J, Mikkelsen T, Wallerman O, Larhammar M, Zhang X, Wang L, Saenz-Vash V, Gnirke A, Lindroth A, Barrés R, Yan J, Strömberg S, De 
S, Pontén F, Lander E, Carr S, Zierath J, Kullander K, Wadelius C, Lindblad-Toh K, Andersson G, Hjälm G, Andersson L: ZBED6, a Novel Transcription Factor Derived from a Domesticated DNA Transposon Regulates IGF2 Expression and Muscle Growth. PLoS Biol 2009, 7:e1000256.

45. Jackson D, McDougall C, Green K, Simpson F, Worheide G, Degnan B: A rapidly evolving secretome builds and patterns a sea shell. BMC Biol 2006, 4:40.

46. Jackson DJ, McDougall C, Woodcroft B, Moase P, Rose RA, Kube M, Reinhardt R, Rokhsar DS, Montagnani C, Joubert C, Piquemal D, Degnan BM: Parallel Evolution of Nacre Building Gene Sets in Molluscs. Mol Biol Evol 2010, 27:591608 .

47. Marie B, Le Roy N, Zanella-Cléon I, Becchi M, Marin F: Molecular evolution of mollusc shell proteins: insights from proteomic analysis of the edible mussel Mytilus. J Mol Evol 2011, 72:531-546.

48. Marie B, Trinkler N, Zanella-Cleon I, Guichard N, Becchi M, Paillard C, Marin F: Proteomic identification of novel proteins from the calcifying shell matrix of the Manila clam Venerupis philippinarum. Mar Biotechnol 2011, 13:955-962.

49. Marie B, Jackson DJ, Ramos-Silva P, Zanella-Cléon I, Guichard N, Marin F: The shell-forming proteome of Lottia gigantea reveals both deep conservations and lineage-specific novelties. FEBS J 2013, 280:214-232.

50. Carroll SB: Evo-devo and an expanding evolutionary synthesis: a genetic theory of morphological evolution. Cell 2008, 134:25-36.

51. Erwin DH, Davidson EH: The evolution of hierarchical gene regulatory networks. Nat Rev Genet 2009, 10:141-148.

52. Wagner G: The developmental genetics of homology. Nat Rev Gen 2007, 8:473479 .

53. Marin F, Luquet G, Marie B, Medakovic D: Molluscan shell proteins: primary structure, origin, and evolution. Curr Top Dev Biol 2007, 80:209-276.

54. Moshel SM, Levine M, Collier JR: Shell differentiation and engrailed expression in the Ilyanassa embryo. Dev Gen Evol 1998, 208:135-141.

55. Jacobs DK, Wray CG, Wedeen CJ, Kostriken R, DeSalle R, Staton JL, Gates RD, Lindberg DR: Molluscan engrailed expression, serial organization, and shell evolution. Evol Dev 2000, 2:340-347.

56. Wanninger A, Haszprunar G: The expression of an engrailed protein during embryonic shell formation of the tusk-shell, Antalis entalis (Mollusca, Scaphopoda). Evol Dev 2001, 3:312-321.

57. Nederbragt AJ, van Loon AE, Dictus WJAG: Expression of Patella vulgata 
orthologs of engrailed and $d p p-B M P 2 / 4$ in adjacent domains during molluscan shell development suggests a conserved compartment boundary mechanism. Dev Biol 2002, 246:341-355.

58. Baratte S, Andouche A, Bonnaud L: Engrailed in cephalopods: a key gene related to the emergence of morphological novelties. Dev Gen Evol 2007, 217:353-362.

59. Iijima M, Takeuchi T, Sarashina I, Endo K: Expression patterns of engrailed and dpp in the gastropod Lymnaea stagnalis. Dev Genes Evol 2008, 218:237-251.

60. Kin K, Kakoi S, Wada H: A novel role for dpp in the shaping of bivalve shells revealed in a conserved molluscan developmental program. Dev Biol 2009, 329:125-166.

61. Shigeno S, Sasaki T, Moritaki T, Kasugai T, Vecchione M, Agata K: Evolution of the cephalopod head complex by assembly of multiple molluscan body parts: Evidence from Nautilus embryonic development. J Morphol 2007, 269:1-17.

62. Hashimoto $\mathrm{N}$, Kurita $\mathrm{Y}$, Wada H: Developmental role of $d p p$ in the gastropod shell plate and co-option of the dpp signaling pathway in the evolution of the operculum. Dev Biol 2012, 366:367-373.

63. Dahmann C, Basler K: Compartment boundaries. Trends Genet 1999, 15:320325.

64. Koop D, Richards G, Wanninger A, Gunter H, Degnan B: The role of MAPK signaling in patterning and establishing axial symmetry in the gastropod Haliotis asinina. Dev Biol 2007, 311:200-212.

65. Shimizu K, Sarashina I, Kagi H, Endo K: Possible functions of Dpp in gastropod shell formation and shell coiling. Dev Genes Evol 2011, 221:59-68.

66. Grande $\mathrm{C}$, Patel N: Nodal signalling is involved in left-right asymmetry in snails. Nature 2009, 457:1007-1011.

67. Kurita Y, Wada $\mathrm{H}$ : Evidence that gastropod torsion is driven by asymmetric cell proliferation activated by TGF- $\beta$ signalling. Biol Lett 2011, 7:759-762.

68. Hinman VF, O'Brien EK, Richards GS, Degnan BM: Expression of anterior Hox genes during larval development of the gastropod Haliotis asinina. Evol Dev 2003, 5:508-521.

69. Samadi L, Steiner G: Involvement of Hox genes in shell morphogenesis in the encapsulated development of a top shell gastropod (Gibbula varia L.). Dev Gen Evol 2009, 219:523-530.

70. Jackson DJ, Wörheide G, Degnan BM: Dynamic expression of ancient and novel molluscan shell genes during ecological transitions. BMC Evol Biol 2007, 7:160. 
71. Richards GS, Simionato E, Perron M, Adamska M, Vervoort M, Degnan B: Sponge genes provide new insight into the evolutionary origin of the neurogenic circuit. Curr Biol 2008, 18:1156-1161.

72. Lai E: Notch signaling: control of cell communication and cell fate. Development 2004, 131:965-973.

73. Barolo S, Posakony JW: Three habits of highly effective signaling pathways: principles of transcriptional control by developmental cell signaling. Genes Dev 2002, 16:1167-1181.

74. Guruharsha K, Kankel M, Artavanis-Tsakonas S: The Notch signalling system: recent insights into the complexity of a conserved pathway. Nat Rev Genet 2012, 13:654-666.

75. Gazave E, Lapébie P, Richards GS, Brunet F, Ereskovsky AV, Degnan BM, Borchiellini C, Vervoort M, Renard E: Origin and evolution of the Notch signalling pathway: an overview from eukaryotic genomes. $\mathrm{BMC} \mathrm{Evol} \mathrm{Biol}$ 2009, 9:249.

76. Arendt D: The evolution of cell types in animals: emerging principles from molecular studies. Nat Rev Gen 2008, 9:868-882.

77. Christodoulou F, Raible F, Tomer R, Simakov O, Trachana K, Klaus S, Snyman H, Hannon GJ, Bork P, Arendt D: Ancient animal microRNAs and the evolution of tissue identity. Nature 2010, 263:1084-1088.

78. Vandesompele J, De Preter K, Pattyn F, Poppe B, Van Roy N, De Paepe A, Speleman F: Accurate normalization of real-time quantitative RT-PCR data by geometric averaging of multiple internal control genes. Gen Biol 2002, 3:research0034. 


\section{Chapter 2}

Hohagen, J. \& Jackson, D. J. (2013). An ancient process in a modern mollusc: early development of the shell in Lymnaea stagnalis.

BMC developmental biology, 13(1), 1-14. 
Chapter 2

\title{
An ancient process in a modern mollusc: early development of the shell in Lymnaea stagnalis
}

\author{
Jennifer Hohagen and Daniel J. Jackson* \\ Courant Research Centre Geobiology, Georg-August University of Göttingen, Goldschmidtstrasse 3, \\ 37077 Göttingen, Germany \\ *Correspondence: djackso@uni-goettingen.de
}

\begin{abstract}
Background: The morphological variety displayed by the molluscan shell underlies much of the evolutionary success of this phylum. However, the broad diversity of shell forms, sizes, ornamentations and functions contrasts with a deep conservation of early cell movements associated with the initiation of shell construction. This process begins during early embryogenesis with a thickening of an ectodermal, 'dorsal' (opposite the blastopore) population of cells, which then invaginates into the blastocoel to form the shell gland. The shell gland evaginates to form the shell field, which then expands and further differentiates to eventually become the adult shell-secreting organ commonly known as the mantle. Despite the deep conservation of the early shell forming developmental program across molluscan classes, little is known about the fine-scale cellular or molecular processes that underlie molluscan shell development.

Results: Using modern imaging techniques we provide here a description of the morphogenesis of a gastropod shell gland and shell field using the pulmonate gastropod, Lymnaea stagnalis as a model. We find supporting evidence for a hypothesis of molluscan shell gland specification proposed over 60 years ago, and present histochemical assays that can be used to identify a variety of larval shell stages and distinct cell populations in whole mounts.

Conclusions: By providing a detailed spatial and temporal map of cell movements and differentiation events during early shell development in L. stagnalis we have established a platform for future work aimed at elucidation of the molecular mechanisms and regulatory networks that underlie the evo-devo of the molluscan shell.
\end{abstract}




\section{Introduction}

Molluscs constitute one of the most successful, morphologically diverse and ancient phyla of the animal kingdom. They posses an extensive fossil record dating back to the early Cambrian (543+ MYA) and comprise more than 200,000 extant species occupying various marine and terrestrial environments from the deep sea to desert habitats $[1,2]$. Much of this evolutionary success can be attributed to the phenotypic plasticity of the external shell, which displays an incredible range of mineralogical textures [3], pigments [4, 5] and ornamentations [6]. This phenotypic diversity is underscored by a diversity in the molecular mechanisms responsible for the construction of the adult shell [7-10].

Despite the morphological and functional-molecular diversity of the adult shell, there is deep conservation of the cellular and morphogenic movements that initiate larval shell secretion (reviewed in [11]). Importantly, larval shell forming cells are thought to give rise to the fully differentiated adult shell forming organ, the mantle, suggesting that trochophore, veliger and adult gastropod shells do not have independent evolutionary origins as previously suggested [12]. Cell lineage studies in disparate gastropods support a common ontogenetic origin of embryonic, larval and adult gastropod shells; derivatives of the 2d and 2c micromeres in Ilyanassa give rise to the shell gland [13], and the same lineage of cells in Crepidula fornicata contributes to the mantle of the veliger [14]. Furthermore, veliger mantle cells expressing shell forming genes continue to do so following metamorphosis in the abalone Haliotis asinina $[15,16]$. The histochemical properties of larval and adult shell forming organs in L. stagnalis also reveal a similar spatial arrangement of enzymatic activities, suggesting that boundaries of shell forming cell populations established in larval stages are maintained into adult life [17]. Additionally, regulatory genes encoding transcription factors and signalling molecules (such as members of the Hox cluster, engrailed and decapentaplegic) are expressed in embryonic shell forming tissue in disparate molluscan taxa [18-25]. This raises the possibility that extant shelled molluscs may all initiate shell formation using the same developmental program inherited from a distant ancestor, and that it is the downstream shell forming programs operating in the mature mantle which during evolution have generated today's shelled diversity of adult molluscs. If such a scenario were true, this would mean that a common ancestor of the shelled molluscs evolved a developmental program to form a shell, which was passed on to all of its 
future descendants; a 540+ million year old innovation that was of great importance to the future evolutionary success of the phylum.

The pulmonate gastropod Lymnaea stagnalis (Linnaeus, 1758) was once a much used model for understanding both molluscan development in general [26] and development of the shell in particular [27]. Development of Lymnaea's shell displays many of the features observed in other gastropod species. Across Molluscan classes, the first morphological sign of shell development is a thickening of the dorsal ectoderm in the post-trochal region of the embryo following gastrulation (see [11] for a review). Briefly, these dorsal ectodermal cells elongate and are often the only ectodermal cells in contact with the underlying endoderm, specifically cells at the tip of the archenteron (the so called 'small-celled endoderm' due to their lack of large vacuoles present in other endodermal cells [26, 28]). These elongated dorsal ectodermal cells then invaginate to form a 'shell gland' $[11,29]$. It is during this stage that secretion of the first shell-associated insoluble material takes place. The shell gland subsequently evaginates to form the 'shell field', a process during which the contact of ectodermal and endodermal cells is lost, and the first signs of calcification of the previously secreted insoluble material can be observed (e.g. [13, $26,27,30]$ ). The initial contact between endoderm and dorsal ectoderm that precedes shell gland invagination has been observed in representatives of the Gastropoda, Bivalvia, Scaphopoda and Cephalopoda (reviewed in [11]). This contact between dorsal ectoderm and endoderm has led to the idea that this event is required for the specification of future shell forming cells, and represents a 'true' induction event [26].

While Raven's model of shell gland induction [26] represents the canonical theory of molluscan shell field specification, the molecular mechanisms that initiate and underlie this process remain largely unknown. Molecular analyses that have previously identified transcription factors and signalling molecules in the shell gland and the evaginated and expanding shell field are expressed well after the specification of shell forming cells $[19,21,24,31]$. We are therefore developing L. stagnalis as a model for molecular investigations into the mechanisms that first specify shell forming cells, and through comparative studies, to enhance our understanding of how the variety of molluscan shells evolved. 
Previous cytological studies on the early development of the shell field in L. stagnalis do not include descriptions of the cellular arrangements preceding the first contact between the dorsal ectoderm and the small-celled endoderm at the tip of the archenteron. Here we employ confocal laser scanning microscopy (CLSM) to provide a detailed temporal and spatial description of the morphogenic events associated with development of the larval shell in L. stagnalis. We have also employed histochemical assays based on endogenous peroxidase (PO) and alkaline phosphatase (AP) activity to identify distinct cell populations within the developing shell gland, shell field and other larval structures in whole mounts. These enzymes are known to be active in the shell forming tissues of several molluscan taxa, including L. stagnalis [17]. These assays allow us to trace discrete cell populations in larval shell forming tissues, and may in the future be employed to characterise the effects of experiments aimed at the perturbation of normal shell development. This work represents a platform from which further studies will investigate the molecular processes leading to the specification and differentiation of molluscan shell forming cells.

\section{Materials and methods}

\section{Cultivation of adult Lymnaea stagnalis}

Adult specimens of L. stagnalis were collected from the Northeimer Seenplatte near Northeim, Germany (51 $\left.43^{\circ} 26.5368 ", 9^{\circ} 57^{\prime} 24.75^{\prime \prime}\right)$ and from a pond on the North campus of the University of Göttingen, Germany (51³3' 23.727", 9० 57' 25.617"). Snails were kept in standard tap water at $25^{\circ} \mathrm{C}$, under a 16:8 light dark regime and fed ad libidum with lettuce and a variety of other vegetables.

\section{Staging and preparation of embryos of L. stagnalis}

Freshly deposited egg masses were collected and their development monitored. Following the first cleavage, egg masses were cultured in snail water [32] at $25^{\circ} \mathrm{C}$. All stages are indicated in hours post first cleavage (hpfc) and days post first cleavage (dpfc). At the desired developmental time point individual egg capsules were removed from an egg mass and freed from the jelly by rolling them over moist filter paper. Embryos were manually dissected from their capsules using forceps 
and needles and fixed according to the subsequent experimental procedure (see below).

\section{Confocal laser scanning microscopy (CLSM)}

29 embryonic stages between 27 and $87 \mathrm{hpfc}$ were fixed at intervals of one to five hours. For each developmental stage, 29 to 126 individuals were visualised, and on average 6 individuals were imaged. To account for fixation artefacts several fixation treatments were tested on embryos between 27 and $37 \mathrm{hpfc}$, ranging from no fixation to extended fixations overnight at room temperature and in varying amounts of gluteraldehyde in combination with a paraformaldehyde-based fixation. Fixation with 4\% paraformaldehyde (PFA) in $1 \mathrm{X}$ phosphate buffered saline (PBS) for one hour at room temperature, or overnight at $4^{\circ} \mathrm{C}$, was found to be optimal. Fixed specimens were washed three times in PBS and processed immediately or stored at $4^{\circ} \mathrm{C}$ for up to five weeks. For cytoplasmic and nuclear staining, samples were incubated in a 1/1000 dilution of Sytox Orange (Molecular probes, S11368) in PBS with $0.1 \%$ TritonX for two hours at room temperature. Samples were then washed three times in PBS, dehydrated through a graded ethanol series and embedded in a 1:2 mixture of benzyl benzoate and benzyl alcohol (BB:BA). Optical sections were captured using a Zeiss LSM 510 Meta with the following settings: HeNe 543 laser at a power of 2.9\%; pinhole between $50 \mu \mathrm{m}$ and $60 \mu \mathrm{m}$ (0.94 to 1.13 Airy Units); amplifier gain of 1; amplifier offset and gain adjusted to the sample brightness; stack size 1024 x 1024 with a stack thickness between $0.81 \mu \mathrm{m}$ and $0.9 \mu \mathrm{m}$; scan speed

and number of scans 7 and 4 or 6 and 8 respectively. For individual images the stack size was 2048 x 2048 with a scan speed and number of 6 and 8 respectively. All images were false-coloured and adjusted for brightness using Macnification version 2.0.1.

\section{Scanning electron microscopy}

Between 55 and 278 embryos for each time point from 27 to $67 \mathrm{hpfc}$ at five hour intervals were fixed in $2.5 \%$ gluteraldehyde in PBS at $4^{\circ} \mathrm{C}$ overnight. These were then dehydrated through a graded ethanol series and dried overnight in hexamethyldisilazane. Samples were mounted on carbon pads on aluminium stubs and sputter-coated with a gold-palladium alloy before being imaged with a scanning electron microscope at $3.8 \mathrm{kV}$. All SEM images were edited in Adobe Photoshop CS3 version 10.0 .1 by applying the 'auto levels' and 'auto contrast' functions. 


\section{Histology and Histochemistry}

The endogenous alkaline phosphatase (AP) activity of 13 developmental stages ranging from 37 to $117 \mathrm{hpfc}$ at 3 to 5 hour intervals was examined. Between 34 and 154 individuals were included in each experiment. Additionally, 14 older larvae (ranging from five days post first cleavage until hatching from the egg capsule) were also assayed for AP activity. For each developmental stage, images of three to 19 individuals were captured. Embryos were fixed for 45 to $60 \mathrm{~min}$ in 4\% PFA in $1 \mathrm{X}$ PBS containing 0.1\% Tween20 (PBTw), and rinsed in $1 \mathrm{X}$ PBS before being incubated in AP reaction buffer (100 mM Tris, $100 \mathrm{mM} \mathrm{NaCl}, \mathrm{pH} 9.5)$ for 5 to $20 \mathrm{~min}$. AP reaction buffer was replaced by detection buffer (AP reaction buffer, $50 \mathrm{mM} \mathrm{MgCl}$, $175 \mu \mathrm{g} / \mathrm{mL}$ BCIP and $450 \mu \mathrm{g} / \mathrm{mL}$ NBT). The colour reaction was stopped after 15 to $60 \mathrm{~min}$ at an optimal signal to background ratio by replacing the detection buffer with $0.1 \mathrm{M}$ Glycine $\mathrm{pH} 2$ containing $0.1 \%$ Tween20. Samples were then rinsed in PBS and post-fixed overnight at room temperature in 4\% PFA in PBS, dehydrated through a graded ethanol series, embedded in BB:BA and viewed and photographed using a Zeiss microscope Axio Imager Z1. A fraction of larger, older (5+ dpfc) larvae were washed twice in PBTw following fixation, and then embedded in $60 \%$ glycerol and imaged under a Zeiss stereo microscope discovery V8.

For developmental stages between $47 \mathrm{hpfc}$ and $5+\mathrm{dpfc}$, the endogenous peroxidase (PO) activity of 45 to 139 individuals was examined prior to performing the AP assay described above in order to visualise the activity of both enzymes at once. Samples fixed as described above were first rinsed twice in $50 \mathrm{mM}$ Tris $\mathrm{pH}$ 7.3, and then pre-incubated in $50 \mathrm{mM}$ Tris $\mathrm{pH} 7.3$ containing $1 \mathrm{mg} / \mathrm{mL}$ diaminobenzidine (DAB) for 15 to 20 min before supplementing the solution with a 1/3000 dilution of $30 \%$ hydrogen peroxide. The colour reaction was monitored and stopped (usually after one to two minutes) by rinsing the samples for about 10 min in $1 \mathrm{X}$ PBS. The AP assay (as described above) was then performed on this material. One to 13 individuals per developmental stage were photo-documented. 


\section{Results}

Using CLSM we have studied the cell arrangements and movements of the embryo from early gastrulation (which precedes any contact between the dorsal ectoderm and the underlying endoderm) until evagination of the shell gland. At $27 \mathrm{hpfc}$ the ventral ectoderm (opposite the future site of the shell field) is broadly depressed representing the initiation of gastrulation (Figs. 1A' and 2A). The proximal ('basal') side of the dorsal ectoderm faces inwards to a large blastocoel cavity (Fig. 2A arrow). Between $29 \mathrm{hpfc}$ and $32 \mathrm{hpfc}$ the invagination of the archenteron initiates and completes. Cells of the archenteron assume an elongated shape from 29 to $35 \mathrm{hpfc}$ (Fig. 2B-E). By 30 hpfc in almost all embryos observed, the endodermal cells at the tip of the archenteron are in contact with the dorsal ectoderm. Initially, this contact does not exist over the entire surface of each cell. Rather, each endodermal cell appears to send out pseudopodia-like projections to the overlying ectoderm (Fig. 2B, boxed region). This results in small spaces being observed between the contacting cellular extensions. In other regions of the embryo, the cells of the invaginating archenteron are separated from the ectoderm by mesodermal cells or intercellular spaces.

The overall shape of the early 29 to $32 \mathrm{hpfc}$ archenteron is slit-like (Figs. 1B' and 2B, C). Between 34 and 37 hpfc the blastopore opening narrows, and the archenteron develops a large round lumen (Figs. 1C' and 2D, E). In most embryos at this stage, a variable number of cells at the tip of the archenteron are in contact with dorsal ectodermal cells directly beneath the large head vesicle cells. By $37 \mathrm{hpfc}$ the contact between endoderm and dorsal ectoderm appears to be firmly established (Fig. 2F). This is the only region in the embryo where these two cell layers are in direct contact with each other, the archenteron is otherwise bordered by mesodermal cells or intercellular spaces. At this stage, neither the ectodermal nor the endodermal cells at the contact site display an altered cell morphology compared with their neighbours using Sytox Orange.

The first signs of differentiation of the dorsal ectoderm cells as observable by CSLM occur at 39 hpfc. At this stage, these cells take on a columnar morphology and are clearly distin-guishable from adjacent ectoderm cells (Fig. 2G). The columnar cells are in direct contact with four to five cells of the tip of the underlying archenteron. 

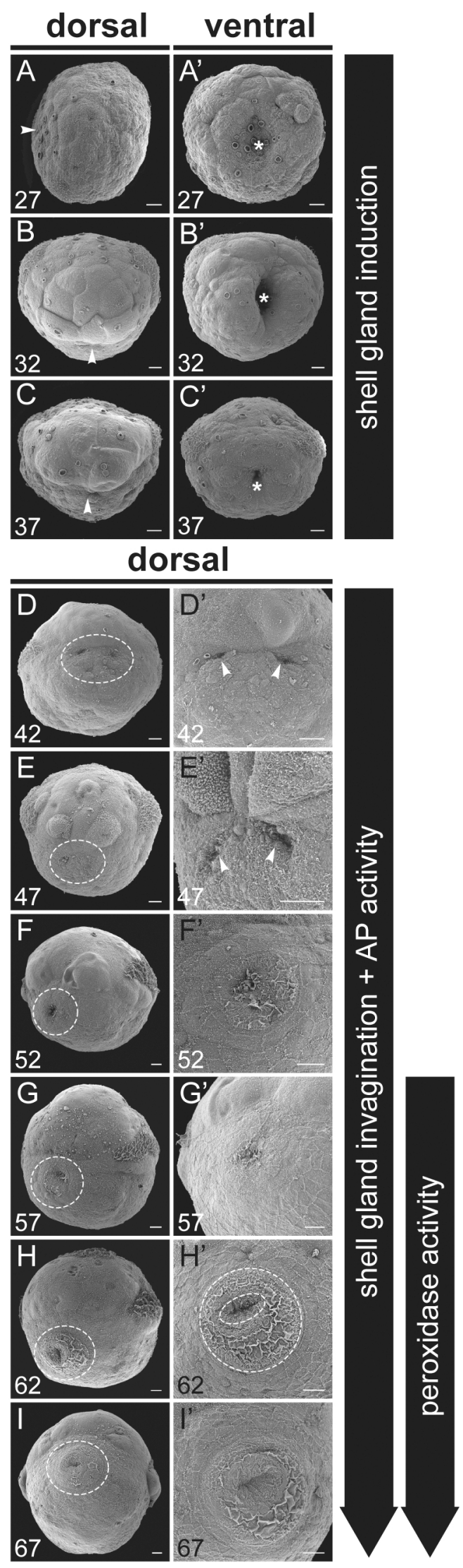

Fig. 1. Early shell development in L. stagnalis illustrated by Scanning Electron Microscopy (SEM). A-C' Gastrulation and for-mation of the archenteron. The site of the future shell gland is marked by white arrowheads. The blastopore is marked by an asterisk. D-E' The first outward signs of shell gland invagination are two shallow depressions at $42 \mathrm{hpfc}$ (arrowheads in D' and E'). F-I' Insoluble material secreted by the shell gland is visible from $52 \mathrm{hpfc}$ onwards. The first asymmetry of the shell is evident at $62 \mathrm{hpfc}$ (highlighted by two dashed ovals in $\mathrm{H}^{\prime}$ ). All scale bars are $10 \mu \mathrm{m}$. Numbers in the lower left of each panel indicate the age in hours post first cleavage. Panel $G$ is reflected about the vertical axis for clarity of presentation.

These endodermal cells in turn are characterised by a lack of large vacuoles that are present in adjacent cells of the archenteron (indicated by ' $x$ 's in Fig. 2G). For this reason these archenteron-tip cells have been referred to as "smallcelled entoderm" [28], here as small celled endoderm. The number of endodermal cells contacting presumptive shell gland cells remains low during the period of contact, never exceeding six cells. During the next hours the morphological differentiation of both cell layers becomes more pronounced with the nuclei of presumptive shell forming cells assuming a basal location (Fig. 2I$\mathrm{K})$. The first external signs of shell gland differentiation are two lateral slit-like depressions that form directly beneath the large head vesicle cells at $42 \mathrm{hpfc}$ (Fig. 1D, D'). 
The first sign of endogenous AP activity can be detected at the same age in the two lateral depressions (Fig. 3A), eight hours earlier than previously reported [17]. In transverse CSLM optical sections, the two lateral depressions of the dorsal ectoderm are first observed at $44 \mathrm{hpfc}$ and deepen in the following hours to form an invaginated shell gland (Fig. 2J-Q). During the invagination process columnar cells at the periphery of the shell gland begin to converge towards each other (Fig. $2 \mathrm{~K}$ arrows). Between $50 \mathrm{hpfc}$ and $52 \mathrm{hpfc}$ the shell gland is comprised of two prominent lateral invaginations and a central elevation (Fig. 2M-0). This bifurcated shell gland morphology is easily visualised by intense AP activity (Fig. 3C, D). Scanning electron micrographs of this stage show a depression surrounded by a concentric arrangement of cells representing the non-invaginated part of the shell gland (Fig. 1F). In the outer-most ring of cells, a second domain of AP activity can be detected which has not been previously reported for Lymnaea. This domain is first visible at $50 \mathrm{hpfc}$ as a semicircle lining the posterior half of the shell gland (Fig. 3C', D'). During the next seven hours the tips of the semi-circle steadily extend anteriorly until a closed ring is formed (white arrows in Fig. 3C', D' and 4A"). From 54 hpfc onwards the central elevated part of the invagination flattens, and the non-invaginated shell gland margins continue to converge (arrows in Fig 2P). By $62 \mathrm{hpfc}$ the margins have converged and the shell gland appears to be a sealed lumen (white dot in Fig. 2Q). All invaginated cells of the shell gland at this stage are AP positive (white dot in Fig. $4 \mathrm{~A}, \mathrm{~B}$ ), with AP activity in non-invaginated cells of the shell gland margin also persisting (white arrows in Fig. 4A).

At this stage, more than ten hours earlier than previously reported [17], the first signs of endogenous PO activity in and around the shell gland are evident. Peroxidase activity can be detected in non-invaginated cells directly adjacent to the shell gland lumen (arrows in Fig. 4A') and adjacent to the peripheral AP positive ring of cells (arrows in Fig. 4A"'), which are not detected in double staining experiments against both enzyme's activities. Also, between $52 \mathrm{hpfc}$ and $57 \mathrm{hpfc}$, the first extra-cellular organic material has been secreted and is stretched over the

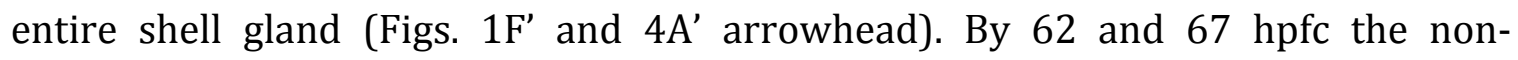
invaginated cells at the periphery of the shell gland are highly elongated (arrow in Fig. 2R). Scanning electron micrographs reveal the shell gland margin as an elevated ring (Fig. $1 \mathrm{H}$ and I'). The secreted insoluble material now lies loosely on the elevated shell gland margin and displays PO activity (arrows in Fig. 4B'). Peroxidase and AP activity also persists in adjacent non-invaginated rings (Fig. 4B" and B"'). The first 
asymmetry in the shell gland is also visible at $62 \mathrm{hpfc}$ with the shell gland slightly shifted to the left side (indicated by the dashed ovals in Fig. 1H'). This asymmetry becomes more pronounced in subsequent stages.
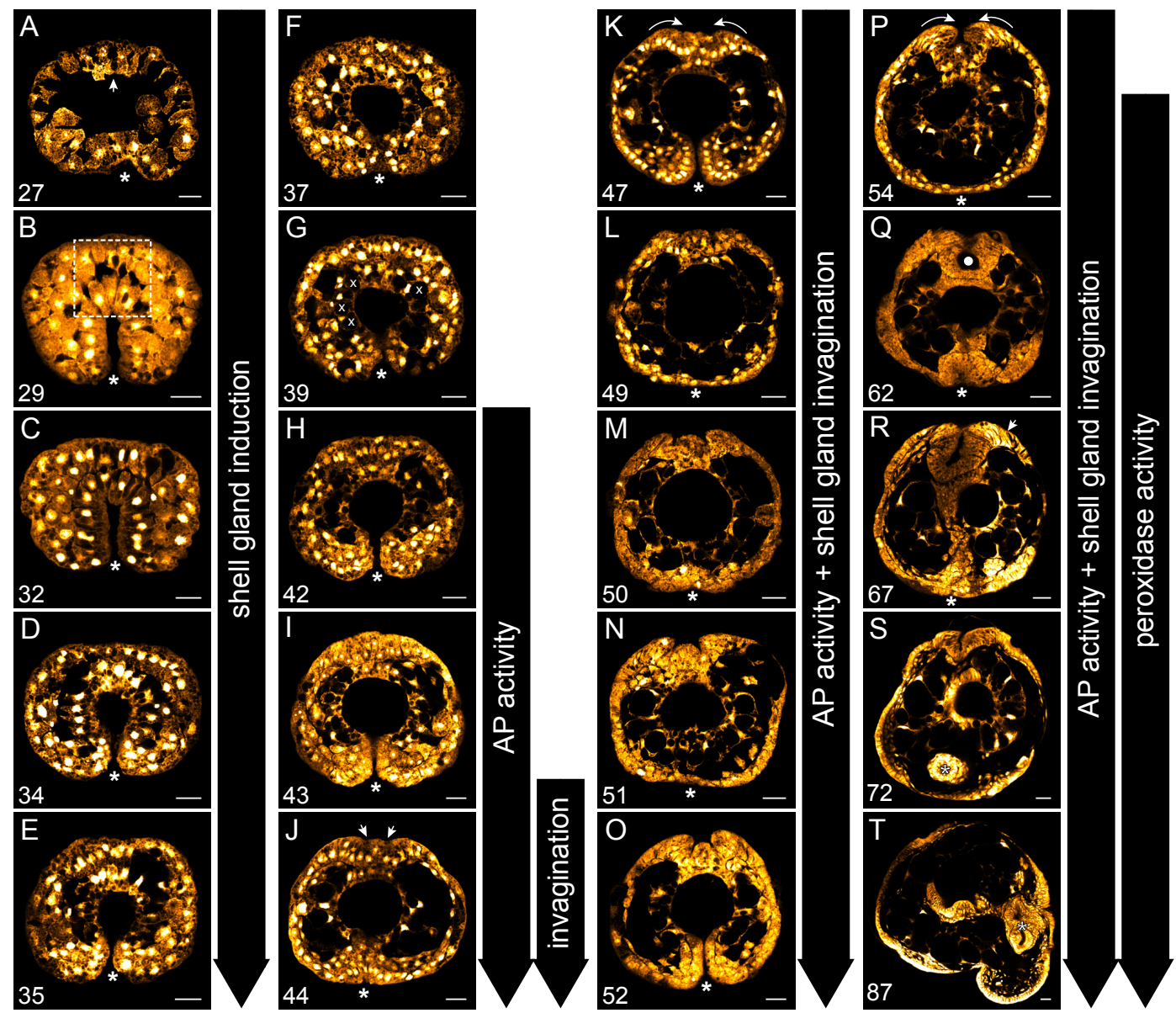

Fig. 2. Early shell development in L. stagnalis illustrated by Confocal Laser Scanning Microscopy (CSLM). A-F During the course of gastrulation the initial ventral invagination (A) deepens and forms the archenteron, which eventually establishes a tight contact with the dorsal ectoderm (F). The initial contact between dorsal ectoderm and endoderm is loose and is characterised by cellular projections (boxed region in B). G-I Upon contact ectoderm and endoderm display signs of differentiation: the dorsal ectodermal cells at the contact zone differentiate into highly columnar shell field cells, and the endodermal cells are characterised by a lack of large vacuoles which are present in adjacent endodermal cells (indicated in G by white " $\mathrm{x}$ "s). J-R The initial bilateral invagination of the shell gland is visible in J (arrows). During this invagination the margins of the shell gland begin to converge (curved arrows in $\mathrm{K}$ and $\mathrm{P}$ ). The non-invaginated margins of the shell gland have converged and form a closed lumen by $62 \mathrm{hpfc}$ (white dot in Q). The cells at the shell gland margin are highly elongated (arrow in R). The embryos in A-S are oriented with the shell field to the top and the embryo in $\mathrm{T}$ is oriented with the shell field to the left. An asterisk marks the position of the blastopore. Panels A-P are transverse optical sections and Panels Q-T are sagittal optical sections. All scale bars are $20 \mu \mathrm{m}$. Numbers in the lower left of each panel indicate the age in hours post first cleavage. 


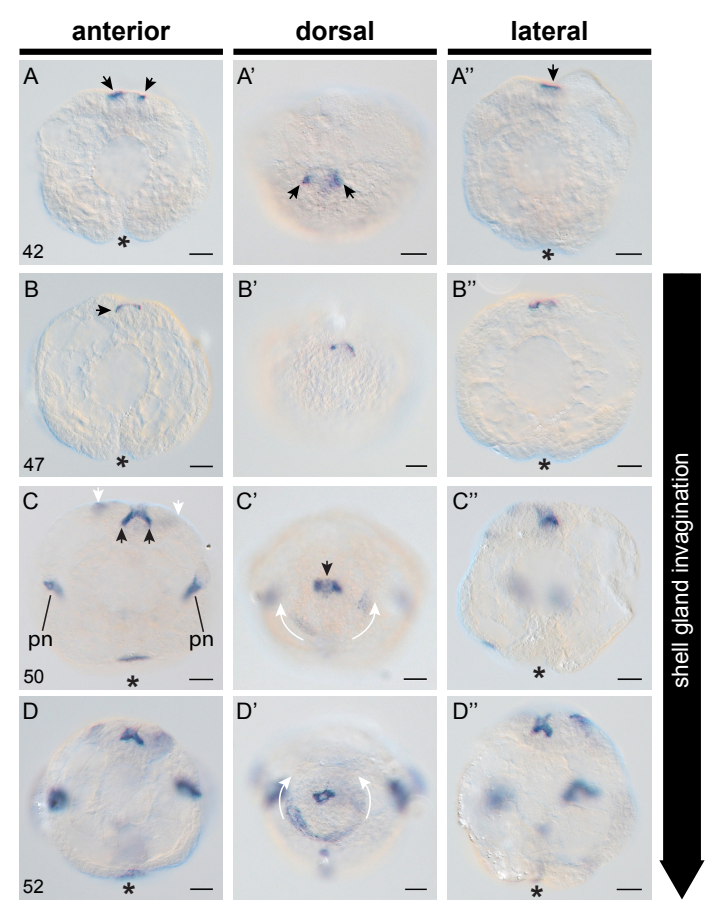

Fig. 3. Alkaline phosphatase (AP) activity in the early shell gland of L. stagnalis.

Endogenous alkaline phosphatase activity (blue) highlights the development of distinct cell populations and structures within the shell gland, and allows for the identification of distinct stages of larval shell development. A$A^{\prime \prime}$ The first evidence of AP activity in the shell gland occurs at $42 \mathrm{hpfc}$ (arrows in A-A"). B-B" Invagination of the shell gland begins at $47 \mathrm{hpfc}$ and this is visible in $\mathrm{AP}^{+}$cells which can be seen just below the outermost level of the dorsal ectoderm (arrow in B). C-C" Noninvaginated $\mathrm{AP}^{+}$cells at the margin of the shell gland (white arrows in C) expand in an anterior direction (curved white arrows in $\mathrm{C}^{\prime}$ ). Invaginated $\mathrm{AP}^{+}$cells (black arrows in $\mathrm{C}$ and $\mathrm{C}^{\prime}$ ) intensify their AP activity. The anlage of the protonephridia (pn) and apical plate become $\mathrm{AP}^{+}$at this stage. All embryos are oriented with the shell field to the top. An asterisk marks the position of the blastopore. All scale bars are $20 \mu \mathrm{m}$. Numbers in the lower left of each panel row indicate the age in hours post first cleavage.

From 67 hpfc onwards the shell gland evaginates giving rise to the shell field. First, the non-invaginated margins of the shell gland diverge, opening up the shell gland lumen (Fig. 2S and curved arrows in Fig. 4C, 5A and 6A"'). Contact between endodermal cells and the dorsal ectodermal is lost at $77 \mathrm{hpfc}$, and the shell field expands in size during subsequent development. Peripheral cells (formerly non-invaginated cells of the shell gland) maintain their columnar shape whereas the central, formerly invaginated cells flatten (Fig. 2T). The relative arrangement of PO and AP activity domains persists from $77 \mathrm{hpfc}$ to $117 \mathrm{hpfc}$. The centre of the shell field displays AP activity with increasing intensity towards the shell field margin (Fig. 5C, C', D'). During the following stages AP activity in the centre of the shell field gradually decreases (Fig. 5C, D). At 117 hpfc AP activity is found in a line of cells proximal to the shell field margin (Fig. 5D). At this stage the secreted organic material and the highly elongated cells of the shell field margin continue to exhibit a strong PO activity (Fig. 5C'” and $\mathrm{D}^{\prime \prime \prime}$ ). In the periphery of the PO positive shell field margin, a faint ring of $\mathrm{AP}^{+}$cells is detectable from $72 \mathrm{hpfc}$ on (Fig. 6). This signal possibly represents the $\mathrm{AP}^{+}$domain of non-invaginated cells seen in earlier stages (see white arrows in Figs. 3 and 4). During the course of shell field differentiation, the activities of both enzymes are continuously located in adjacent, non-overlapping cell 
populations within the shell field (Fig. 6A-C'”; see Fig. 8 for a schematic summary).

\section{Non-shell related $A P$ and $P O$ activities during larval development}

Endogenous AP and PO activities can also be used to follow the development of larval structures in L. stagnalis in a more general way. Several structures besides the

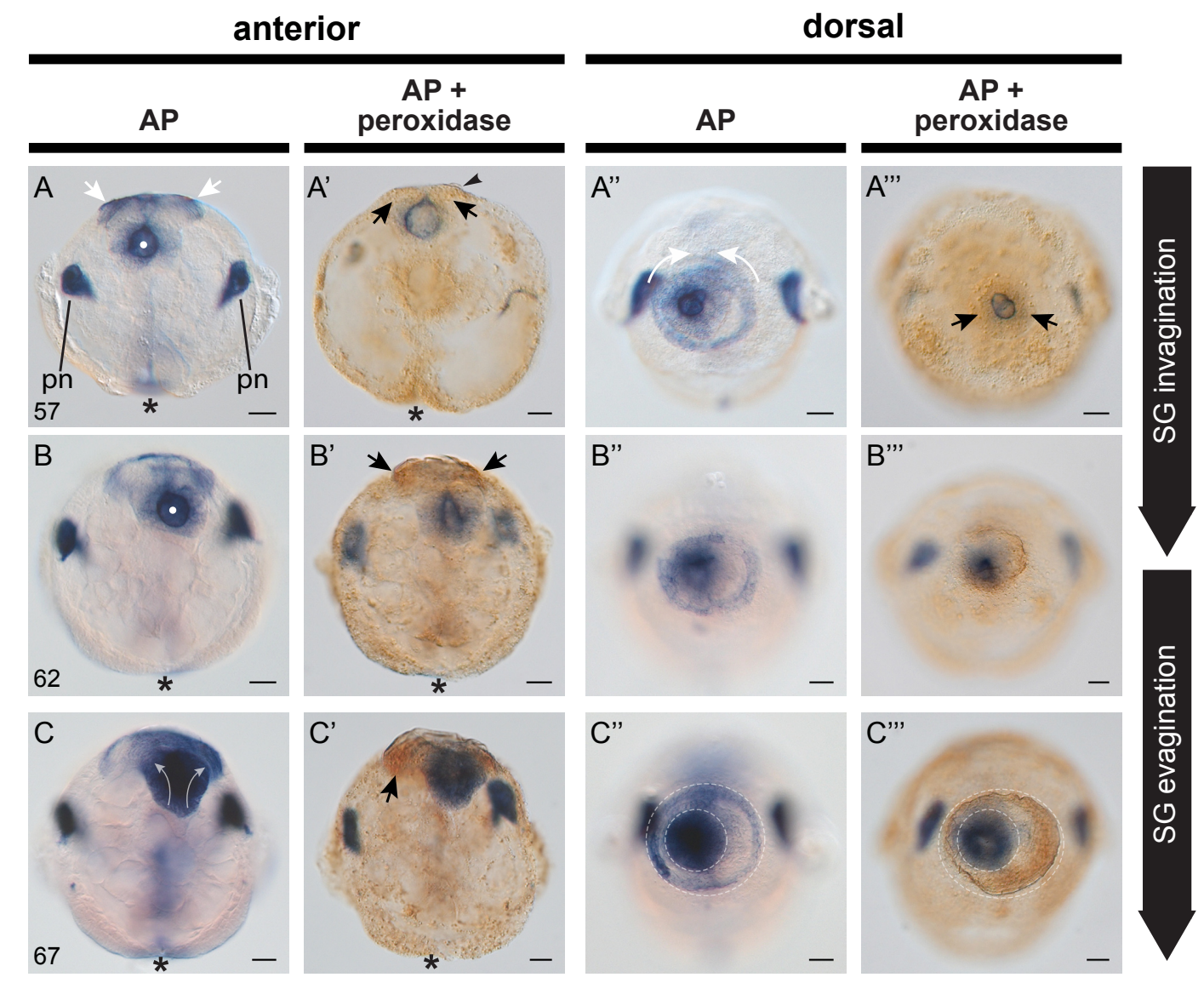

Fig. 4. Alkaline phosphatase (AP) and peroxidase (P0) activity in the mature shell gland of $L$. stagnalis. Endogenous AP activity intensifies as the shell gland matures, and PO activity also becomes detectable. A-A"' The mature shell gland at $57 \mathrm{hpfc}$ is characterised as a closed lumen with intense AP activity (white dot in A). AP activity has also increased in non-invaginated cells at the margin of the shell gland (white arrows in A), and in the anlage of the protonephridia (pn). Weak PO activity is also evident at this stage (black arrows in $\mathrm{A}^{\prime}$ and $\mathrm{A}^{\prime \prime}$ ), and secreted insoluble material can also be seen in preparations of this age (arrowhead in $\mathrm{A}^{\prime}$ ). Non-invaginated $\mathrm{AP}^{+}$cells at the margin of the shell gland finish their anterior expansion, meeting at the midline (curved white arrows in $\mathrm{A}^{\prime \prime}$ ). $\mathbf{B}$ B'"' In $62 \mathrm{hpfc}$ larvae the shell gland is maintained as a closed lumen (white dot in B) while PO activity in non-invaginated cells intensifies (arrows in B'). C-C'" Between 62 and 67 hpfc evagination of the shell gland has started (transparent arrows in $\mathrm{C}$ ) and PO activity in non-invaginated cells of the shell gland margin has also increased (arrow in $\mathrm{C}^{\prime}$ ). The asymmetry of the shell gland is made clearly visible by populations of $\mathrm{AP}^{+}$and $\mathrm{PO}^{+}$cells (dashed ovals in $\mathrm{C}^{\prime \prime}$ and $\mathrm{C}^{\prime \prime \prime}$ respectively). All embryos are oriented with the shell field to the top. An asterisk marks the position of the blastopore. All scale bars are $20 \mu \mathrm{m}$. Numbers in the lower left of each panel row indicate the age in hours post first cleavage. 


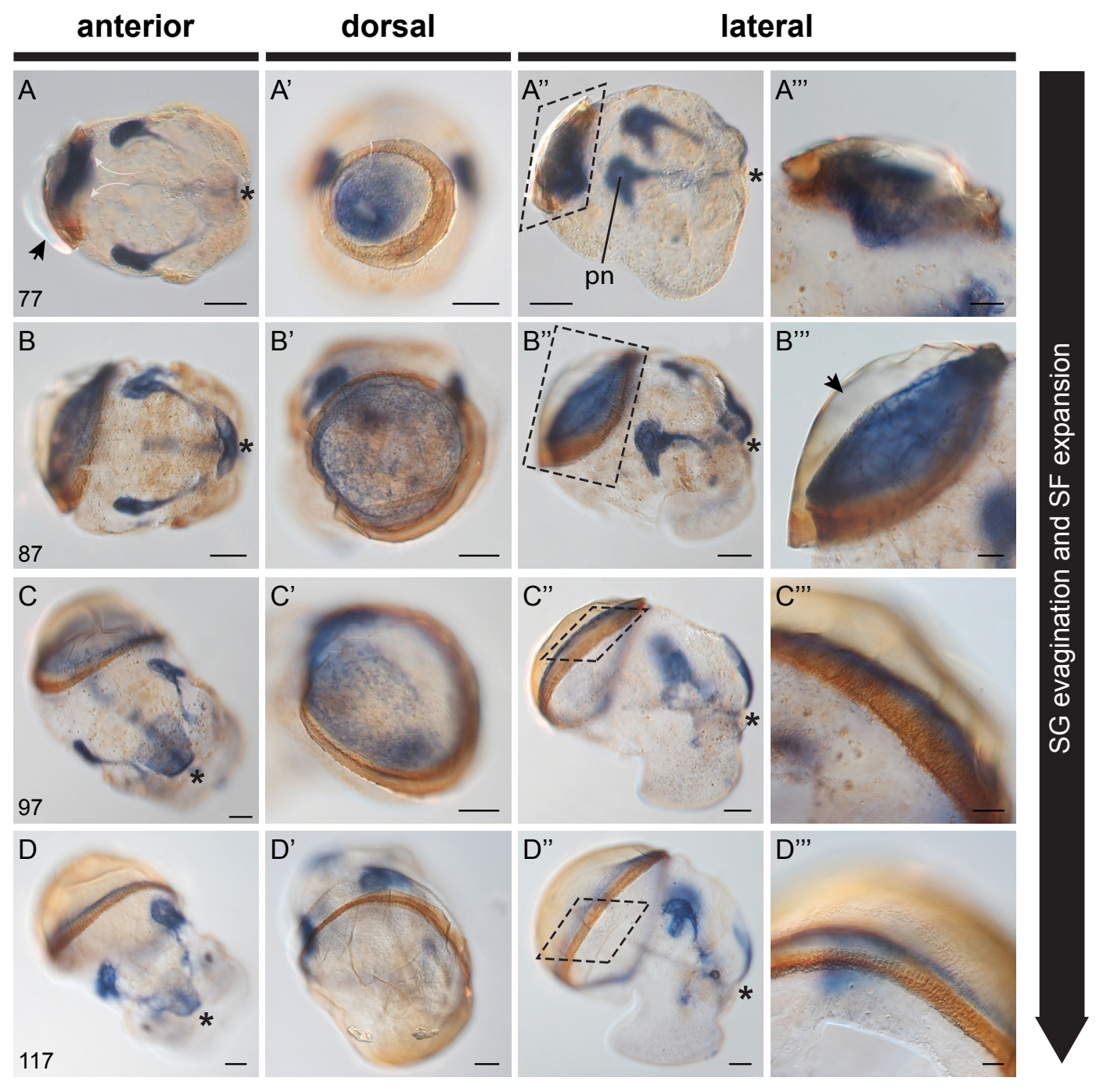

Fig. 5. Alkaline phosphatase (AP) and peroxidase (PO) activity in the evaginating shell gland and expanding shell field of $\boldsymbol{L}$. stagnalis. All panels are double labelled for AP and PO activity. A-A'" By $77 \mathrm{hpfc}$ secreted birefringent material (arrow in A) overlying the evaginating shell gland is clearly visible. The shell gland is no longer a closed lumen (cf. Fig. 4A and 4B) as it continues to evaginate (indicated by transparent arrows in A). A ring of intense PO activity now surrounds a field of AP activity $\left(\mathrm{A}^{\prime}\right)$. The protonephridia (pn) remain intensely $\mathrm{AP}^{+}\left(\mathrm{A}^{\prime \prime}\right)$. B-B'”' By $87 \mathrm{hpfc}$ shell gland evagination appears to be complete and the field of $\mathrm{AP}^{+}$cells in the shell gland has expanded in all directions ( $\mathrm{B}^{\prime}$ ). A sheet of organic material overlies the shell field (arrow in B'"). C-C'" At 97 hpfc the shell field continues to increase in size and the border between $\mathrm{PO}^{+}$and $\mathrm{PO}^{-}$cells at the shell field margin sharpens (C'”). D-D'” By 117 hpfc AP activity in the shell gland is concentrated in cells directly adjacent to $\mathrm{PO}^{+}$cells. The embryos are oriented with the shell field to the left. An asterisk marks the position of the stomodaeum. Scale bars are $20 \mu \mathrm{m}$ (A'", B'", C'"' and D"') or $50 \mu \mathrm{m}$ (A-A", BB", C-C" and D-D"). Numbers in the lower left of each panel row indicate the age in hours post first cleavage. Panel A" is reflected about the vertical axis for clarity of presentation. 


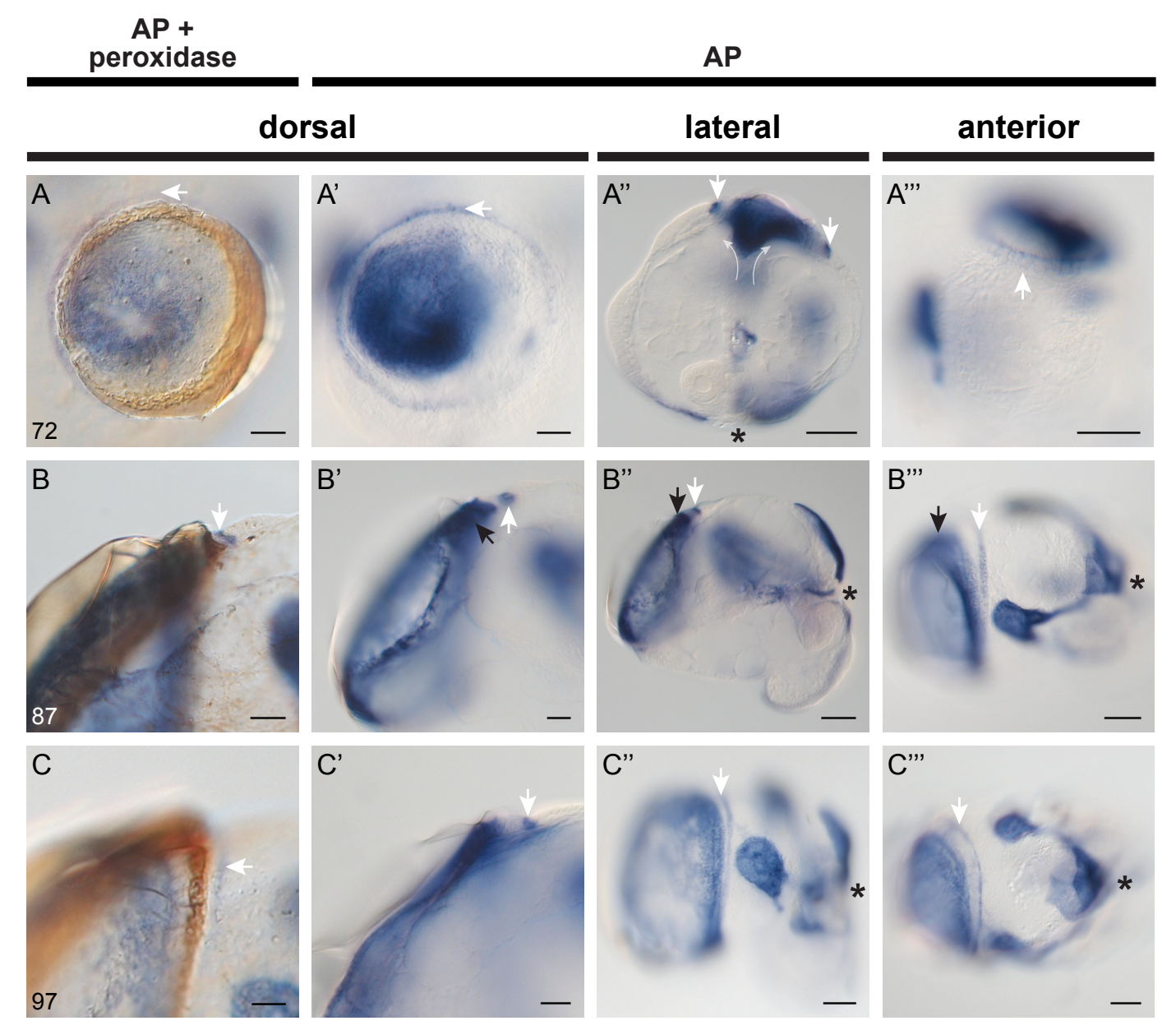

Fig. 6. Multiple alkaline phosphatase (AP) domains reveal a differentiated and complex organisation of the shell gland. A second domain of AP activity present within the shell gland and shell field (not described by Timmermans [17]), is located outside of the PO domain and can not be clearly detected after prior detection of PO activity (see Fig. 5 and Fig. 6A cf. 6A'). A-A"' This second $\mathrm{AP}^{+}$domain associated with non-invaginated cells of the shell gland margin can first be detected at $50 \mathrm{hpfc}$ (see white arrow in Fig. 3C), and is more pronounced at around $72 \mathrm{hpfc}$ (white arrows in A$\mathrm{A}^{\prime \prime \prime)}$. This $\mathrm{AP}^{+}$domain subsequently decreases in strength. B-B'"' At $87 \mathrm{hpfc}$ the distinct domains of AP activity associated with the evaginating shell gland (black arrows) and the non-invaginated cells of the shell gland margin (white arrows) are still visible. C-C"' By 97 hpfc the domain of AP activity associated with non-invaginated cells of the shell gland margin is considerably weaker (white arrows). It's position relative to $\mathrm{PO}^{+}$cells can be seen clearly in $\mathrm{C}$ (white arrow). The embryos in $\mathrm{A}-\mathrm{A}^{\prime \prime \prime}$ are oriented with the shell field to the top, the embryos in B-C'” are oriented with the shell field to the left. An asterisk marks the position of the stomodaeum. Scale bars are $20 \mu \mathrm{m}$ (A-A', B-B' and C-C') or $100 \mu \mathrm{m}$ (A"-A"', $B^{\prime \prime}-B^{\prime \prime \prime}$ and C'-C'"). Numbers in the lower left of each panel row indicate the age in hours post first cleavage. Panels A", A"', B", C and C'" are reflected about the vertical axis for clarity of presentation. 

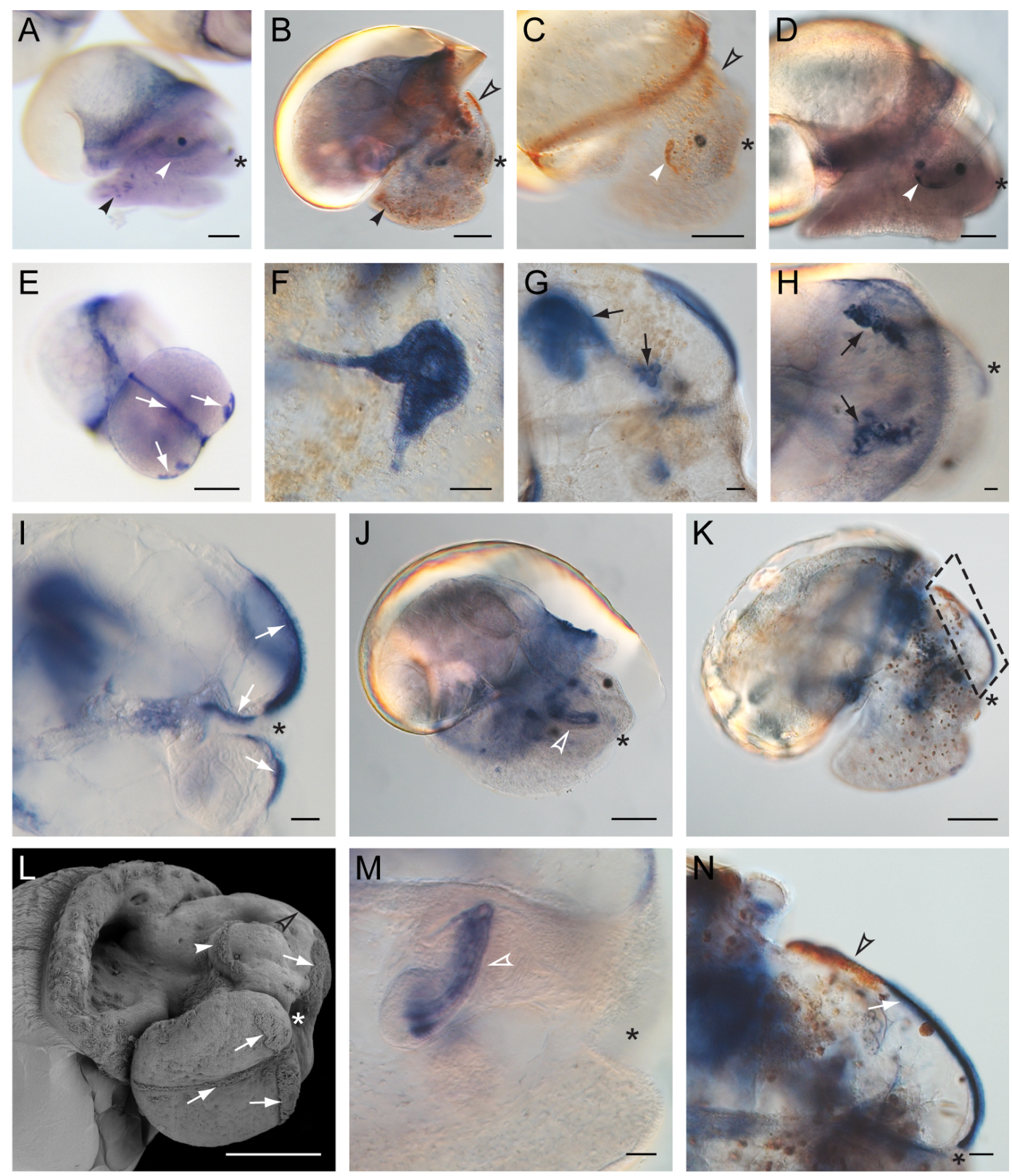

Fig. 7. Endogenous alkaline phosphatase (AP) and peroxidase (P0) activities as markers of larval development. Other organs and cell populations not involved in shell formation display AP and PO activity. Endogenous AP activity can be found in most ciliated fields (E and I, white arrows), including the apical plate (G, I, K, N), the protonephridia (F-H, black arrows), the developing radula ( $\mathrm{J}$ and $\mathrm{M}$, white open arrowhead) and dispersed cells throughout the foot (A, black arrowhead). Peroxidase positive structures include the head vesicle cells ( $\mathrm{B}, \mathrm{C}, \mathrm{K}$ and $\mathrm{N}$, black open arrowheads), the velum (C, white arrowhead) and scattered cells throughout the foot and head tissue (B, black arrowhead, and $\mathrm{K}$ respectively). $\mathrm{N}$ is a detailed view of the boxed area in $\mathrm{K}$. L is an SEM from a lateral/ventral perspective of a 5+ dpfc staged larva illustrating the ciliated fields of the foot and head (white arrows) and the velum (white arrow head). A-D, H, J and M show larvae of $5+d p f c$, F shows a 77 hpfc old larva, I shows a 97 hpfc old larva and E, G, K and N show 117 hpfc old larvae. The position of the stomodaeum is marked by an asterisk. Scale bars are $20 \mu \mathrm{m}$ (F-I, M and N) or $100 \mu \mathrm{m}$ (A-E, J, K, L). Panels B, G, I, K, and N are reflected about the vertical axis for clarity of presentation. 
shell gland and shell field display endogenous activity of these enzymes (Fig. 7). AP activity is present in most ciliated fields (Fig. 7E, I, K and N) and the protonephridia (Fig. 7F-H) [26]. In older 5+ dpfc stages the developing radula (Fig. 7J and M) and cells throughout the foot exhibit AP activity (Fig.7A). Endogenous PO activity can be found in ectodermally derived cells scattered over the head and foot region (Fig.7B and $\mathrm{K}$ ) and later in the head vesicle cells anterior from of the apical plate (Fig. 7B, C, $\mathrm{K}$ and $\mathrm{N}$ ) [33]. Both enzymes show adjacent, but non-overlapping activity in the head region and activity in the velum [33] (Fig. 7C and D).

\section{Discussion}

In various molluscan groups, the initial differentiation of the dorsal shell forming ectoderm has been observed to coincide with the presence of a tight contact with underlying endodermal cells (reviewed in [11]). These observations raised the possibility that this contact is required for the specification of molluscan shell forming cells in general. Based on manipulative experiments, first Raven [26] and later Hess $[34,35]$ concluded that it is this dorsal ectoderm/endoderm contact that specifies the shell field. Raven differentiates between two possibilities of contactdependent shell field specification, induction vs. activation. Raven [26] realised that if the dorsal ectoderm is truly induced to become the shell gland by such a contact (rather than activated as would be the case if a population of dorsal ectodermal cells were already specified in someway), two morphogenic preconditions must be realised. Firstly, only a restricted part of the endoderm (i.e. cells at the tip of the archenteron) should be able to elicit this specification in the overlying ectoderm. Secondly, the whole ectoderm (or at least the majority) should be able to respond to this induction by forming a shell gland. Raven's observations based on four embryos (whose gastrulation had been perturbed by lithium and which developed an ectopic shell gland) lead Raven to conclude that most of the ectoderm is indeed able to respond to signals from the underlying endoderm, and that therefore the dorsal ectoderm is truly induced by the endoderm to become shell forming tissue, rather than activated. This model of induction-mediated shell field specification has since been supported [34], modified [13,35] and contradicted [36-38]. These studies report a wide capacity of the ectoderm to form a shell field, but disagree about the origin of the "inductive cue". Hess's observations of partial embryos after 
blastomere separation in Bythinia and L. stagnalis [34,35] support the contact dependent model of shell field specification, but indicate that any endodermal tissue, even single cells, is capable of induction. Cell deletion experiments in Ilyanassa [13] support the hypothesis that there is no cellular specificity in the inducing endoderm: all combinations of ectoderm and endoderm can generate a shell field. Furthermore, in Ilyanassa it has been shown that the tip of the archenteron is never in close proximity to the dorsal ectoderm [37]. Labordus and van der Wal [38] extending the studies on Ilyanassa by Clement [36] and Cather [13], suggest a scenario, which distinguishes between the histogenic and morphogenic differentiation of the shell gland. Based on observations of misdeveloped embryos producing internal shell material, Labordus and van der Wal [38] propose that the histogenic differentiation necessary to produce such material is independent of inductive interactions, whereas the correct spatial organisation of shell forming tissues depends on spatially correct inductive interactions between the D-quadrant macromeres and the overlying micromeres earlier in development. Based on that study, McCain [39] conducted cell deletion experiments that suggest inductive interactions among the micromeres are also required to give rise to the larval shell forming tissues. The disturbance of these interactions by the removal of participating cells leads to the internal deposition of calcium carbonate similar to those observed by earlier workers, supporting the assumption that the processes leading to the histogenetic vs. the morphogenetic differentiation of larval shell forming tissues do not depend on each other. This hypothesis is further corroborated by work in experimental systems that allow for an artificially induced shell internalisation, e.g. by exposure to environmental toxins such as platinum [40, 41]. In these systems (Marisa cornuarietis and Planorbis corneus), platinum interferes with the localisation of shell material and the growth of shell forming tissues, while the cellular differentiation of these tissues appears to remain unaffected [40,41].

While Raven's hypothesis of induction-mediated shell gland specification still represents the most comprehensive theory of how the future shell forming cells are initially specified in molluscs, contradictory observations have been reported for a number of disparate taxa. In the pulmonate taxa Bradybaena and Achatina marginata the shell field is differentiated before any contact with the underlying endoderm is established, in the Caenogastropod Marisa this contact is interrupted by intermingled cells, and in other gastropod species (Ilyanassa obsoleta and Achatina fulica) as well as bivalves (Cyclas and Sphaerium) no contact is present 
(reviewed in [11]). Unfortunately, none of these previous studies utilised high resolution imaging techniques such as CLSM that are available today, or conducted their investigations with high temporal resolution. Nonetheless we must acknowledge that there does exist the possibility of an alternative, contactindependent shell gland specification mechanism, leaving open the question as to whether the dorsal ectoderm/endoderm contact event represents the ancestral molluscan mode of shell gland specification.

While the present study was not intended to clarify the molecular mechanisms of shell gland specification, nor to differentiate between scenarios of induction vs. activation as proposed by Raven [26], it does clarify the nature of the cellular interactions between endoderm and ectoderm prior to and during shell gland specification, and also provides an accurate framework for the timing of these events in L. stagnalis at $25^{\circ} \mathrm{C}$.

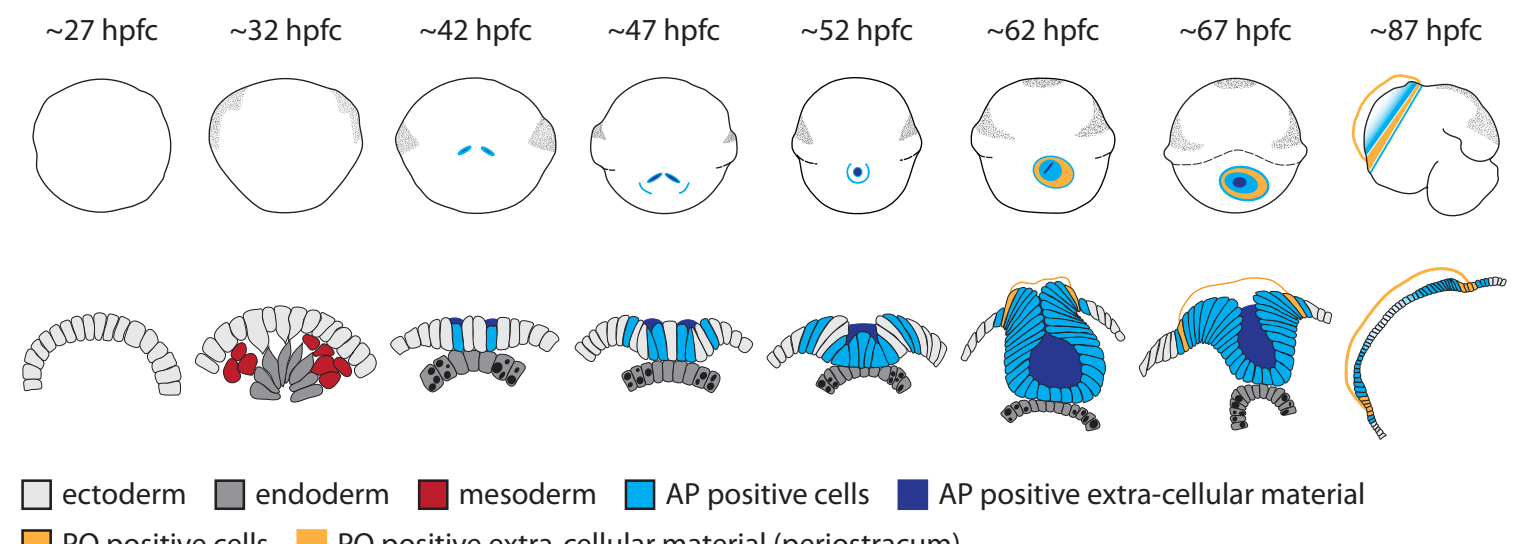

PO positive cells $\quad$ PO positive extra-cellular material (periostracum)

Fig. 8. Schematic representation of the major events during early development of the shell in $L$. stagnalis. Uppermost row are all dorsal views except for $\sim 87 \mathrm{hpfc}$ which is a lateral view. Lowermost row are the corresponding transverse sections through the developing shell gland and shell field. By approximately 32 hours post first cleavage (hpfc) endodermal cells at the tip of the archenteron (the future so called "small celled entoderm" for their lack of large vacuoles indicated by black ovals and circles) have made contact with the overlying cells of the dorsal ectoderm. These are the only endodermal cells to make contact with ectoderm. By $42 \mathrm{hpfc}$ cells that are in contact with the endoderm have thickened and some cells display alkaline phosphatase (AP) activity. The strongest AP activity is apparently in extra-cellular material. A bilateral invagination of the shell gland has also commenced at this age. By 47 hpfc the bilateral invagination has deepened and noninvaginated, posterior shell gland cells at the periphery of the shell gland also display AP activity. At 52 hpfc the bilateral invaginations have fused, and the lumen of the shell gland displays intense AP activity. By 62 hpfc the lumen of the shell gland appears to be sealed with intense AP activity. The first peroxidase (PO) activity is visible at this time and is evident in cells and in the secreted periostracum material. By $87 \mathrm{hpfc}$ the form of the juvenile snail has been established and the nonoverlapping zones of AP and PO activity are maintained. 
Using CSLM we could reconstruct the cellular arrangements and movements during contact between dorsal ectoderm and endoderm. Despite its importance, former studies do not include a description of how this contact is initially established. If, as Raven [26] proposes, the 'small-celled endoderm' truly induces the overlying dorsal ectoderm in Lymnaea, these endodermal cells should have acquired their inductive capacity prior to contact. Such prior differentiation is not revealed by Sytox Orange staining in our study (both dorsal ectoderm and the 'small-celled endoderm' show the first signs of differentiation after contact establishment; see Fig. 2F and G), nor by Raven using standard histological stains [26]. Raven concluded that acquisition of an inductive capacity by the endoderm is not revealed by any histological differentiation. Indeed, a molecular differentiation of the contacting endoderm could be expected to precede any visible histological differentiation. Identification of such molecular markers would provide great insight into the evolution of the molluscan shell.

Our study also reveals a pronounced bilateral organisation of early shell gland development; the invagination of the shell gland begins when two lateral points of the thickened dorsal ectoderm form two lateral depressions (Fig. 1D-E', 2J-0). This bilateral organisation persists until the margins of the shell gland converge above the lumen of the invaginated shell gland and the bifurcated lumen rounds up (Figs. 1F', 2P-R). The formation of this shell gland lumen coincides with the secretion of the first insoluble shell material. None of the invaginated shell gland cells appear to participate in the secretion of this first water insoluble material, which emerges from the peripheral non-invaginated shell gland cells [27] (Fig. 1F'). This observation has raised the hypothesis that the process of shell gland invagination is required in order to bring cells at the periphery of the shell gland into close contact, and to thereby initiate the secretion of an insoluble shell forming matrix without a central hole above the shell gland lumen [42, 43]. Shortly afterwards, the first signs of asymmetry in the shell gland appear. The invaginated part of the shell gland shifts to the left side, which generates a larger distance between the centre of the lumen and the peripheral secreting cells on the right side than on the left side (Figs. 1H', 4C', C'”). This early asymmetry presumably reflects the future coiling direction of the mature shell.

While shell gland formation is a deeply conserved feature of molluscan development, there is considerable diversity in its ontogeny within and between all molluscan 
groups. For those species with internal or reduced shells, the formation and further differentiation of the shell gland differs from that seen in L. stagnalis and other externally shelled molluscs (reviewed in [11]). In shell-less cephalopods for example, shell gland development ceases during dorsal ectoderm invagination, and an evaginated shell field never forms. In cephalopods with an internal shell, the shell gland is internalised and characterised by a closed pore, and is therefore referred to as a "shell sac". This structure is not thought to be formed by an invagination of the central part of the thickened dorsal ectoderm. Instead, the peripheral cells of the thickened dorsal ectoderm bulge upwards and overgrow the central cells. An internalised shell gland or "shell sac" is also found in shell-less terrestrial slugs, but is formed by a different mechanism. Here, the dorsal ectoderm invaginates as it does in shelled gastropods, but continues inwards leading to a complete internalisation of all shell gland cells, and consequently a closure of the shell gland. These ontogenetic events in secondarily shell-less slugs also illustrate their common ancestry with shelled snails such as Lymnaea.

\section{Endogenous enzyme activities as a tool to illustrate larval development}

Our study demonstrates the usefulness of endogenous AP and PO activity as markers to map molluscan development. During the course of shell gland and shell field differentiation both enzymes are continuously located in distinct shell forming cells (summarised in Fig. 8). Larval structures such as ciliated fields and the protonephridia are also AP positive, and the head vesicles in older embryos display PO activity (Fig. 7). Previous studies of AP activity during shell field development in L. stagnalis were based on acetone-fixed and paraffin-embedded sections, a procedure that results in a significant loss of enzyme activity [17]. Using the methods we describe here, we can detect both earlier and novel domains of AP and PO activity, and can also simultaneously detect AP and PO activities. AP and PO activity in shell forming tissues has been shown for a number of gastropod and bivalve taxa (summarised in [17]). Transcripts encoding these enzyme families derived from developmental stages and/or adult shell-secreting tissues can be found in sequence databases for divergent molluscan taxa, suggesting that these enzymes might have conserved functions during molluscan shell development. While the precise function of AP in molluscan shell forming tissues has not been described, AP activity in vertebrate bone (hydroxy apatite) forming tissues is known to regulate levels of inorganic pyrophosphate, a potent inhibitor of mineralisation 
$[44,45]$. PO activity is displayed by non-invaginated cells at the periphery of the shell gland. These cells appear to be intimately associated with the production of the periostracum which is itself $\mathrm{PO}^{+}$(Figs. 4, 5, 6). It has been suggested that peroxidases in the periostracum may assist in the crosslinking of periostracal proteins, rendering them insoluble and resistant to abrasion [17, 27]. These simple histochemical assays provide a tool not only to identify and trace functionally distinct cell populations within the developing shell gland and shell field, but also to simply assist with the orientation of the molluscan embryo. In the future, such assays could be used to assess the effects of manipulative experiments such as shellspecific gene knock-down assays.

\section{Conclusions}

This work represents a platform from which analyses aimed at the identification of the molecular regulators responsible for shell development in L. stagnalis can be conducted. We have described the timing of developmental events critical to specification of shell forming cells, and the movements of cells that take part in these processes. We also highlight the use of histochemical assays that allow for the detection of endogenous alkaline phosphatase and peroxidase activity within shell forming cells. Understanding the molecular basis of shell development from a range of molluscan representatives will provide deep insight into the evolutionary events that supported the generation of much of today's molluscan diversity. The work presented here is a first step towards the development of L. stagnalis as a model to understand how this diversity arose.

\section{Acknowledgements}

We gratefully acknowledge Luciana Macis for expert technical assistance and maintenance of snail lines, and Dorothea Hause-Reitner for capturing the SEM images. Nora Glaubrecht assisted with initial Sytox Orange CLSM experiments. This work was supported by Deutsche Forschungsgemeinschaft (DFG) funding to DJJ through the CRC for Geobiology and the German Excellence Initiative, and DFG project number JA 2108/1-1. 


\section{References}

1. Ponder WF, Lindberg DR: Phylogeny and Evolution of the Mollusca. Berkeley: University of California Press; 2008.

2. Brusca RC, Brusca GJ: Invertebrates. Sunderland: Sinauer Associates Incorporated; 2002.

3. Lowenstam HA, Weiner S: On biomineralization. New York: Oxford University Press, Inc.; 1989.

4. Comfort A: The pigmentation of molluscan shells. Biol Rev 1951, 26:285301.

5. Meinhardt H: The Algorhithmic Beauty of Sea Shells. Berlin Heidelberg: Springer; 2009.

6. Abbott RT, Dance SP: Compendium of Seashells: a Full-color Guide to More Than 4,200 of the World's Marine Shells. El Cajon: Odyssey Publishing; 1998.

7. Marin F, Luquet G, Marie B, Medakovic D: Molluscan shell proteins: primary structure, origin, and evolution. Curr Top Dev Biol 2007, 80:209-276.

8. Jackson DJ, McDougall C, Woodcroft B, Moase P, Rose RA, Kube M, Reinhardt R, Rokhsar DS, Montagnani C, Joubert C, Piquemal D, Degnan BM: Parallel Evolution of Nacre Building Gene Sets in Molluscs. Mol Biol Evol 2010, 27:591-608.

9. Marie B, Le Roy N, Zanella-Cléon I, Becchi M, Marin F: Molecular evolution of mollusc shell proteins: insights from proteomic analysis of the edible mussel Mytilus. J Mol Evol 2011, 72:531-546.

10. Marie B, Jackson DJ, Ramos-Silva P, Zanella-Cléon I, Guichard N, Marin F: The shell-forming proteome of Lottia gigantea reveals both deep conservations and lineage-specific novelties. FEBS J 2013, 280:214-232.

11. Kniprath E: Ontogeny of the Molluscan Shell Field: a Review. Zool Script 1981, 10:61-79.

12. Haszprunar G, von Salvini-Plawen L, Rieger RM: Larval planktotrophy - a primitive trait in the Bilateria? Acta Zool 1995, 76:141-154.

13. Cather JN: Cellular Interactions in the Development of the Shell Gland of the Gastropod, Ilyanassa. J Exp Zool 1967, 166:205-223.

14. Hejnol A, Martindale MQ, Henry JQ: High-resolution fate map of the snail Crepidula fornicata: The origins of ciliary bands, nervous system, and muscular elements. Dev Biol 2007, 305:63-76. 
15. Jackson DJ, Ellemor N, Degnan BM: Correlating gene expression with larval competence, and the effect of age and parentage on metamorphosis in the tropical abalone Haliotis asinina. Mar Biol 2005, 147:681-697.

16. Jackson DJ, Wörheide G, Degnan BM: Dynamic expression of ancient and novel molluscan shell genes during ecological transitions. BMC Evol Biol 2007, 7:160.

17. Timmermans LPM: Studies on shell formation in molluscs. Neth J Zool 1969, 19:63-78.

18. Jacobs DK, Wray CG, Wedeen CJ, Kostriken R, DeSalle R, Staton JL, Gates RD, Lindberg DR: Molluscan engrailed expression, serial organization, and shell evolution. Evol Dev 2000, 2:340-347.

19. Wanninger A, Haszprunar G: The expression of an engrailed protein during embryonic shell formation of the tusk-shell, Antalis entalis (Mollusca, Scaphopoda). Evol Dev 2001, 3:312-321.

20. Nederbragt AJ, van Loon AE, Dictus WJAG: Expression of Patella vulgata orthologs of engrailed and dpp-BMP2/4 in adjacent domains during molluscan shell development suggests a conserved compartment boundary mechanism. Dev Biol 2002, 246:341-355.

21. Hinman VF, O'Brien EK, Richards GS, Degnan BM: Expression of anterior Hox genes during larval development of the gastropod Haliotis asinina. Evol Dev 2003, 5:508-521.

22. Iijima M, Takeuchi T, Sarashina I, Endo K: Expression patterns of engrailed and dpp in the gastropod Lymnaea stagnalis. Dev Genes Evol 2008, 218:237251.

23. Kin K, Kakoi S, Wada H: A novel role for $\boldsymbol{d p p}$ in the shaping of bivalve shells revealed in a conserved molluscan developmental program. Dev Biol 2009, 329:125-166.

24. Samadi L, Steiner G: Involvement of Hox genes in shell morphogenesis in the encapsulated development of a top shell gastropod (Gibbula varia L.). Dev Gen Evol 2009, 219:523-530.

25. Hashimoto N, Kurita Y, Wada H: Developmental role of $d p p$ in the gastropod shell plate and co-option of the dpp signaling pathway in the evolution of the operculum. Dev Biol 2012, 366:367-37.

26. Raven CP: Morphogenesis in Limnaea stagnalis and its disturbance by lithium. J Exp Zool 1952, 121:1-77. 
27. Kniprath E: Zur Ontogenese des Schalenfeldes von Lymnaea stagnalis. Dev Gen Evol 1977, 181:11-30.

28. Raven CP: The Development of the Egg of Limnaea Stagnalis L. From the First Cleavage Till the Trochophore Stage, With Special Reference To Its" Chemical Embryology". Arch Neer Zool 1946, 7:353-434.

29. Wassnig M, Southgate PC: Embryonic and larval development of Pteria penguin (Röding, 1798)(Bivalvia: Pteriidae). J Moll Stud 2012, 78:134-141.

30. Bielefeld U, Becker W: Embryonic development of the shell in Biomphalaria glabrata (Say). Int J Dev Biol 1991, 35:121-131.

31. Shimizu K, Sarashina I, Kagi H, Endo K: Possible functions of Dpp in gastropod shell formation and shell coiling. Dev Genes Evol 2011, 221:5968.

32. Thomas JD, Lough AS, Lodge RW: The Chemical Ecology of Biomphalaria glabrata (Say), the Snail Host of Schistosoma mansoni Sambon: The Search for Factors in Media Conditioned by Snails which Inhibit their Growth and Reproduction. J Anat 1975, 12:421-436.

33. Morrill JB: Development of the Pulmonate Gastropod, Lymnaea. In Developmental Biology of Freshwater Invertebrates. Edited by Harrison FW, Cowden RR. New York: Alan R. Liss, Inc.; 1982:399-483.

34. Hess O: Die Entwicklung von Exogastrulakeimen bei dem SüsswasserProsobranchier Bithynia tentaculata L. Dev Gen Evol 1956, 148:474-488.

35. Hess O: Die Entwicklung von Halbkeimen bei dem Süsswasserpulmonaten Limnaea stagnalis L. Dev Gen Evol 1957, 150:124-145.

36. Clement AC: Development of Ilyanassa following removal of the D macromere at successive cleavage stages. J Exp Zool 1962, 149:193-215.

37. Collier JR, McCann-Collier M: Shell gland formation in the Ilyanassa embryo. Exp Cell Res 1964, 34:512-514.

38. Labordus V, van der Wal UP: The determination of the shell field cells during the first hour in the sixth cleavage cycle of eggs of Ilyanassa obsoleta. J Exp Zool 1986, 239:65-75.

39. McCain ER: Cell interactions influence the pattern of biomineralization in the Ilyanassa obsoleta (Mollusca) embryo. Dev Dyn 1992, 195:188-200.

40. Osterauer R, Marschner L, Betz O, Gerberding M, Sawasdee B, Cloetens P, Haus $\mathrm{N}$, Sures B, Triebskorn R, Köhler H: Turning snails into slugs: induced body plan changes and formation of an internal shell. Evol Dev 2010, 12:474-483. 
41. Marschner L, Triebskorn R, Köhler $\mathrm{H}$ : Arresting mantle formation and redirecting embryonic shell gland tissue by platinum2+ leads to body plan modifications in Marisa cornuarietis (Gastropoda, Ampullariidae). J Morphol 2012, 273:830-841.

42. Kniprath E: The functional morphology of the embryonic shell-gland in the conchiferous molluscs. Malacologia 1979, 18:549-552.

43. Eyster LS: Ultrastructure of early embryonic shell formation in the opisthobranch gastropod Aeolidia papillosa. Biol Bull 1983, 165:394-408.

44. Hessle L, Johnson KA, Anderson HC, Narisawa S, Sali A, Goding JW, Terkeltaub R, Millán JL: Tissue-nonspecific alkaline phosphatase and plasma cell membrane glycoprotein-1 are central antagonistic regulators of bone mineralization. Proc Natl Acad Sci U S A 2002, 99:9445-9449.

45. Harmey D, Hessle L, Narisawa S, Johnson KA, Terkeltaub R, Millán JL: Concerted Regulation of Inorganic Pyrophosphate and Osteopontin by Akp2, Enpp1, and Ank: An Integrated Model of the Pathogenesis of Mineralization Disorders. Am J Pathol 2004, 164:1199-1209.

\section{Credits}

I carried out all experimental procedures. I participated in the design of the study by choosing the investigated time points and proposing some of the applied methods. I drafted the manuscript and contributed many of the aspects presented in the discussion.

Daniel J. Jackson conceived and supervised the study. He participated in the design of the study by choosing some of the applied methods. He participated in drafting the manuscript and contributed most of the ideas stated in the introduction and some of the discussed aspects. 


\section{Chapter 3}

Hohagen J., Herlitze I. \& Jackson, D. J. (in review) A whole mount in situ hybridisation protocol for the re-emerging molluscan model Lymnaea stagnalis

BMC developmental biology 


\title{
A whole mount in situ hybridisation protocol for the re-emerging molluscan model Lymnaea stagnalis
}

\author{
Jennifer Hohagen, Ines Herlitze and Daniel J. Jackson* \\ Courant Research Centre Geobiology, Georg-August University of Göttingen, Goldschmidtstrasse 3, \\ 37077 Göttingen, Germany \\ *Correspondence: djackso@uni-goettingen.de
}

\begin{abstract}
Background: The ability to visualise the expression of individual genes in situ represents an invaluable tool for developmental and evolutionary biologists; it allows for the characterisation of processes such as gene function, gene regulation and (commonly through inter-specific comparisons) the evolutionary history of morphological novelties. For well-established model organisms (e.g., flies, worms, sea urchins) this technique has been optimised to an extent where it can be automated for high-throughput analyses. While the overall concept of in situ hybridisation is simple (hybridise a single-stranded, labelled nucleic acid probe complementary to the target of interest, and then detect the label colourimetrically or fluorescently), there are many modifiable parameters in the technique that can significantly affect the quality of the final result. Furthermore, due to variation in the biochemical and biophysical characteristics of organic substrates an in situ technique optimised for one species is often not suitable for another, and may even vary depending on the ontogenetic stage or targeted gene within a species. Here we report the systematic optimisation of a whole mount in situ hybridisation (WMISH) technique for the re-emerging molluscan model Lymnaea stagnalis. The phylogenetic position of L. stagnalis in the Tree of Life is significant because it is a representative of a vastly understudied clade of morphologically diverse animals characterised by a spiral arrangement of the first several cell divisions, the presence of a trochophore-like larva and/or a lophophore (the Spiralians/Lophotrochozoans). Results: Using a variety of pre-hybridisation treatments we have identified a set of conditions that greatly increases both WMISH signal intensity and consistency while
\end{abstract}


maintaining morphological integrity for different developmental stages of L. stagnalis. These treatments function well for a set of genes with presumably significantly different levels of expression ( $\beta$-tubulin, engrailed and COE). We also identify tissuespecific background staining in the larval shell field of L. stagnalis as well as a treatment, which eliminates this signal.

Conclusions: The set of optimised in situ hybridisation methods that we present here will allow researchers to more readily adopt L. stagnalis as an informative model for evo-devo studies, and represents a much needed addition to the relatively short list of tractable molluscan models. 


\section{Introduction}

Analyzing how spatial and temporal developmental gene expression profiles evolve is a powerful strategy for understanding how morphological diversity can be generated. The most commonly employed technique for the study of spatial gene expression in a given tissue or developmental stage is in situ hybridisation, often implemented by evolutionary-developmental biologists on developmental stages as whole mount in situ hybridisation (WMISH). WMISH provides information about the timing and localization of a gene's expression in a developing embryo or larva, and can be used to characterise and identify cell types, tissues or organs within the whole organism and to make inferences about their function and evolutionary history, e.g. [1-3]. However, the technique is challenging, especially when applied to an organism for which there is little previous knowledge regarding the multifarious conditions that optimise the balance between WMISH signal intensity and the preservation of morphological integrity, two often conflicting requirements. WMISH experiments on embryos can be further challenged by changes in the biochemical and biophysical properties of the developing tissues during ontogenesis. Thus, the procedure often needs to be adapted for distinct developmental stages.

From an evo-devo perspective, the pulmonate freshwater gastropod Lymnaea stagnalis (Linnaeus, 1758) is a representative of a significantly understudied group of animals, the Spiralia/Lophotrochozoa. Primarily due to its availability and ease of culture, L. stagnalis was once a much used model for studying molluscan development [4-6], and is currently experiencing a resurgence in popularity as a model for evo-devo studies focused on various biological processes including the establishment of chirality [7], the evolution of shell formation [8] and ecologically regulated development [9]. However, L. stagnalis possesses certain traits that represent serious technical challenges to a WMISH method. First, L. stagnalis embryos develop individually within egg capsules filled with a fluid that serves a nutritive function and is uptaken by pinocytosis during development [10-12]. This viscous intra-capsular fluid, which consists of a complex mixture of ions, polysaccharides, proteoglycans and other polymers [13], can be seen to stick to the embryo following decapsulation, and is very likely to interfere with the WMISH procedure. Second, from 52 hours post first cleavage onwards the first insoluble material associated with shell formation is secreted [8]. This material non- 
specifically binds nucleic acid probes and generates a characteristic background signal. Finally, L. stagnalis embryos and larvae undergo significant morphometric and biophysical changes in the characteristics of their tissues during the first days of development which has implications for the permeabilisation treatments required to provide the labelled probe with access to its target.

In order to achieve consistent WMISH signals with minimal background in L. stagnalis larvae, we have systematically compared the influence of a variety of chemical and enzymatic treatments selected to overcome each of these challenges. Briefly, the mucolytic agent $\mathrm{N}$-acetyl-L-cysteine (NAC) has been shown to improve WMISH signal intensity in the platyhelminth flatworm Schmidtea mediterranea, most likely by degrading the mucosal layer surrounding the animal and thereby increasing tissue permeabilisation [14]. Tissue permeabilisation for WMISH was further improved in S. mediterranea through the use of a combination of detergents and the reducing agent dithiothreitol (DTT) [14]. Here we assess the impact of different combinations of these and other standard WMISH treatments (fixation, storage, enzymatic permeabilisation, acetylation) on the strength and consistency of the WMISH signal across a variety of larval stages in L. stagnalis. We have performed these experiments with a selection of three genes, which can be reasonably assumed to have different levels of expression: $\beta$-tubulin, and the transcription factors engrailed and COE (collier/olfactory-1/early B cell factor). We also demonstrate the presence of a shell field-specific background stain, which can be abolished by treatment with triethanolamine (TEA) and acetic anhydride. The optimised WMISH method we present here allows for future molecular studies to be performed on a wide range of developmental processes within L. stagnalis.

\section{Materials and methods}

Cultivation of adult L. stagnalis and preparation of embryos

A laboratory culture derived from adult L. stagnalis collected from the Northeimer Seenplatte, Germany (51 $\left.43^{\prime} 26.5368^{\prime}, 9^{\circ} 57^{\prime} 24.75^{\prime}\right)$ and from a pond on the North campus of the University of Göttingen, Germany (51 $33^{\prime} 23.727^{\prime}, 9^{\circ} 57^{\prime} 25.617^{\prime}$ ) were kept in standard tap water at $25^{\circ} \mathrm{C}$, under a $16: 8$ light dark regime and fed $a d$ libidum with lettuce and a variety of other vegetables. Under this regime adult snails 
lay egg masses year round. Egg masses of diverse ages were collected and grouped into two developmental time windows: from approximately two to three days post first cleavage ( $\mathrm{dpfc}$ ) and from three to six dpfc. Individual egg capsules were freed from the surrounding jelly by rolling them over moist filter paper. Embryos were released from their egg capsules by manual dissection using forceps and mounted needles. In order to maximize the comparability of treatment effects, cohorts of embryos were processed in batches until receiving their unique treatments.

\section{NAC treatment}

Freshly dissected embryos were immediately incubated in a solution of NAC in 1X PBS. The duration and concentration of this treatment were age-dependent. Embryos ranging from two to three dpfc were treated for five minutes with $2.5 \%$ NAC, samples between three and six dpfc age were treated with 5\% NAC twice for five minutes each. All samples were immediately fixed after NAC treatment.

\section{Fixation}

All samples were transferred into freshly prepared $4 \%(\mathrm{w} / \mathrm{v}$ ) paraformaldehyde (PFA) in 1X PBS and allowed to sit for five minutes followed by gentle rotation for 20 minutes. All samples were then allowed to settle without agitation for 5 minutes. The fixative was then removed by one five minute wash in 1X PBTw. Samples were then either subjected to the reduction treatment described below or dehydrated through a graded ethanol (EtOH) series; one wash in $50 \%$ (v/v) EtOH, two washes in $100 \% \mathrm{EtOH}$, each wash lasting five to ten minutes. All samples were then stored at $-20^{\circ} \mathrm{C}$.

\section{Reduction}

Embryos between two and three dpfc were treated with 0.1X reduction solution for ten minutes at room temperature. Embryos between three and six dpfc were incubated for ten minutes in preheated $1 \mathrm{X}$ reduction solution at $37^{\circ} \mathrm{C}$. All samples were carefully inverted once during this time. After removal of the reduction solution, all samples were briefly rinsed with PBTw before being dehydrated through a graded EtOH series; one wash in $50 \%(\mathrm{v} / \mathrm{v}) \mathrm{EtOH}$, two washes in $100 \%$ $\mathrm{EtOH}$, each wash lasting five to ten minutes. All samples were then stored at $-20^{\circ} \mathrm{C}$. 
RNAse treatment in order to investigate the source of non-specific WMISH staining Following fixation, dehydration and storage at $-20^{\circ} \mathrm{C}$ samples were rehydrated through a graded EtOH series into PBTw and were then incubated for 30 minutes at $37^{\circ} \mathrm{C}$ in $10 \mu \mathrm{g} / \mathrm{mL}$ and $100 \mu \mathrm{g} / \mathrm{mL}$ RNAse A (Sigma, \#R5503) in 2X SSC. Samples were then washed five times in PBTw for 5 minutes each before proceeding with Pro-K digestion.

\section{Protein digestion with Proteinase-K (Pro-K)}

Following fixation, dehydration, storage at $-20^{\circ} \mathrm{C}$ and any additional treatments (RNAse, bleach or reduction), samples were rehydrated through a graded EtOH series into PBTw. Embryos were then treated with an age-dependent concentration of Pro-K (Carl Roth, \#7528) for ten minutes at room temperature. The regimes presented here follow a more exhaustive series of trials using a greater range of Pro-K concentration. Embryos between two and three dpfc were incubated in concentrations of Pro-K ranging from 10-20 $\mu \mathrm{g} / \mathrm{mL}$, and older embryos (between three and six dpfc) were treated at concentrations of 20 and $30 \mu \mathrm{g} / \mathrm{mL}$. Pro-K activity was stopped by two five minutes washes in $2 \mathrm{mg} / \mathrm{mL}$ glycine. All samples were then briefly rinsed in PBTw.

\section{Triethanolamine + acetic anhydride (TEAAA) treatment}

Samples were transferred into a $1 \%(\mathrm{v} / \mathrm{v})$ solution of triethanolamine (TEA) in PBTw and incubated for five minutes. This step was then repeated. This solution was then replaced with a solution of $1 \%$ TEA $+0.3 \%(\mathrm{v} / \mathrm{v})$ acetic anhydride (TEAAA) in PBTw. After five minutes the solution was renewed and the samples were incubated for an additional five minutes. All samples were then washed three times with PBTw, post-fixed for ten minutes in 4\% PFA in PBTw, and washed three times with PBTw before being transferred into an Intavis In situ-Pro robot for all subsequent hybridisation, antibody incubation and washing steps.

\section{Riboprobes synthesis}

Primers designed to amplify fragments of $\beta$-tubulin, engrailed and COE were designed from 454 and Illumina RNASeq data (see Table 1 for all primer sequences). These PCR products were cloned into vectors containing T7 and SP6 promoter sites and verified by Sanger sequencing. These fragments were then amplified from plasmid DNA using M13 primers, and purified using the QIAGEN QIAquick Gel 
Extraction Kit. Antisense riboprobes were synthesized using Promega reagents in a $10 \mu \mathrm{L}$ reaction containing $1 \mathrm{X}$ reverse transcription buffer, $10 \mathrm{mM}$ Dithiothreitol, 1X Digoxigenin RNA labelling Mix (Roche, \#11277073910), 0.25 - 0.5 volume PCR template and 20 Units of the appropriate RNA polymerase (SP6 or T7; Promega, \#P108 or \#P207). Probe synthesis reactions were carried out at $37^{\circ} \mathrm{C}$ for two to four hours. For $\beta$-tubulin, a 702 bp long internal fragment was used for riboprobe synthesis. For engrailed, a 929 bp internal fragment partially covering the homeobox domain was used. The riboprobe against $C O E$ was generated from a $1626 \mathrm{bp}$ long internal fragment covering the DNA binding domain and the TIG/IPT domain was used. All riboprobes were purified by precipitation using 0.1 volume of $3 \mathrm{M}$ sodium acetate $\mathrm{pH} 5.2$ and 3 volumes of absolute EtOH for 15 minutes, and subsequently centrifuged for 15 minutes at 16,000 RCF. All precipitation steps were carried out at room temperature. The resulting pellets were washed once in $75 \%$ $\mathrm{EtOH}$, dried and dissolved in $20 \mu \mathrm{L}$ water at $55^{\circ} \mathrm{C}$. After quantification using a Nanodrop, between $500 \mathrm{ng}$ and $1 \mu \mathrm{g}$ of riboprobe was denatured in $95 \%$ formamide at $75^{\circ} \mathrm{C}$ for 10 minutes and qualitatively assessed by agarose gel electrophoresis.

\section{Hybridisation and antibody binding}

All samples were incubated in hybridisation buffer for 15 minutes at room temperature before being heated to the hybridisation temperature of $55^{\circ} \mathrm{C}$. The hybridisation buffer was then exchanged and incubated for an additional two hours. Riboprobe in hybridisation buffer was denatured for ten minutes at $75^{\circ} \mathrm{C}$ using a water bath, and aliquoted into individual hybridisation reaction tubes for subsequent use in the robot. The hybridisation buffer on all samples was replaced by the riboprobe in hybridisation buffer and allowed to hybridise for ten hours at $55^{\circ} \mathrm{C}$ using the following optimised concentrations of riboprobes: $\beta$-tubulin $150 \mathrm{ng} / \mathrm{mL}$; engrailed $500 \mathrm{ng} / \mathrm{mL}$; COE $200 \mathrm{ng} / \mathrm{mL}$ or $300 \mathrm{ng} / \mathrm{mL}$. Unbound probe was washed out with three washes in $4 \mathrm{X}$ wash buffer for 15 minutes, three washes in $2 \mathrm{X}$ wash buffer for 15 minutes, three washes in $1 \mathrm{X}$ wash buffer for 15 minutes and one wash in $1 \mathrm{X} \mathrm{SSC}+0.1 \%$ Tween, all performed at $55^{\circ} \mathrm{C}$. Samples were then allowed to cool to room temperature and then washed twice in 1X SSC $+0.1 \%$ Tween for 15 minutes. Two washes in maleic acid buffer (MAB) pH 7.5 were then performed for ten minutes each. All samples were then cooled to $10^{\circ} \mathrm{C}$ and incubated for three hours in pre-cooled block solution with one exchange. Block solution was then replaced by block solution containing a 1:10,000 dilution of anti-DIG 
antibody (Roche, \#11093274910) and incubated for five hours followed by a renewal of this solution and a further five hour incubation, all at $10^{\circ} \mathrm{C}$. Unbound antibody was removed by ten washes of PBTw for ten minutes each, and five subsequent washes with $\mathrm{PBS}$ at $10^{\circ} \mathrm{C}$.

\section{Colour development and post-processing}

For each gene, trial colour development reactions were performed on a small fraction of each sample in order to determine the optimal duration of colour development. This amount of time was then used to develop the WMISH signal in all remaining samples and to therefore make inter-treatment comparisons meaningful. For colour development samples were transferred into $1 \mathrm{X}$ alkaline phosphatase buffer with $0.1 \%(\mathrm{v} / \mathrm{v})$ Tween 20 (APTw) and incubated with two ten minutes washes at room temperature. The 1X APTw was replaced by the detection buffer and colour development was performed in the dark for the length of time determined by the trial colour reactions. The colour reaction was stopped by replacing the colour substrate solution by $0.1 \mathrm{M}$ Glycine $\mathrm{pH} 2$. After approximately five minutes, samples were then rinsed in PBTw and post-fixed in 4\% (v/v) PFA in PBTw for two hours at room temperature or over night at $4^{\circ} \mathrm{C}$. The fixative was removed by two washes with PBTw, followed by two washes with pre-warmed deionised water for each ten minutes at $37^{\circ} \mathrm{C}$. Embryos were then dehydrated through a graded EtOH series (once with 50\%, twice with 100\%) and stored at $-20^{\circ} \mathrm{C}$.

\section{Imaging}

Samples were rehydrated into PBTw with one intermediate 50\% EtOH step, and cleared at $4^{\circ} \mathrm{C}$ over night in $60 \%$ (v/v) glycerol. For "bulk" imaging (where an image of tens of embryos gives an impression of the reproducibility of a given treatment) embryos were mounted in a 96 well plate with U-shaped bottom and imaged under a Zeiss stereo Discovery V8 microscope. For images of individual embryos, samples were mounted on glass slides and photographed using a Zeiss microscope Axio Imager Z1. Images of individual embryos were also captured at different focal planes some of which were projected using Macnification version 2.0.1. All images were edited in Adobe Photoshop CS3 version 10.0.1 by adjusting the entire image for brightness and contrast. 


\section{Solutions}

1X PBS (phosphate buffered saline): 0.1 volume of 10X PBS stock $(1.37 \mathrm{M} \mathrm{NaCl}$; $27 \mathrm{mM} \mathrm{KCl} ; 100 \mathrm{mM} \mathrm{Na}_{2} \mathrm{HPO}_{4} \cdot 2 \mathrm{H}_{2} \mathrm{O} ; 20 \mathrm{mM} \mathrm{KH}_{2} \mathrm{PO}_{4}$ ).

1X PBTw (Phosphate buffered saline + Tween 20): 0.1 volume of 10X PBS stock; $0.1 \%(\mathrm{v} / \mathrm{v})$ Tween-20.

2.5\% NAC ( $\mathrm{N}$-acetyl cysteine): 0.5 volume of $5 \%(\mathrm{w} / \mathrm{v})$ NAC in $1 \mathrm{X}$ PBS.

4\% PFA (paraformaldehyde): 0.25 volume of $16 \%$ (w/v) PFA pH 7-8; 1X PBS or 1X PBTw.

50\% EtOH (ethanol): 0.5 volume of absolute EtOH in water.

0.1X reduction solution: $0.1 \%(\mathrm{v} / \mathrm{v}$ ) Tergitol (NP40); $0.05 \%(\mathrm{v} / \mathrm{v})$ sodium dodecyl sulfate (SDS); $5 \mathrm{mM}$ dithiothreitol.

1X reduction solution: 1\% (v/v) Tergitol (NP40); 0.5\% (v/v) SDS, $50 \mathrm{mM}$ dithiothreitol.

Pro-K (Proteinase-K): Diluted from $10 \mathrm{mg} / \mathrm{mL}$ stock using PBTw.

2 mg/mL glycine pH 2: Diluted from $100 \mathrm{mg} / \mathrm{mL}$ stock using PBTw.

TEA (triethanolamine): $1 \%(\mathrm{v} / \mathrm{v}$ ) TEA diluted in $1 \mathrm{X}$ PBTw.

TEAAA (triethanolamine + acetic anhydride): $1 \%(\mathrm{v} / \mathrm{v})$ TEA; $0.3 \%(\mathrm{v} / \mathrm{v})$ acetic anhydride diluted in 1x PBTw.

Hybridisation buffer: 0.25 volume 20X SSC stock $(3 \mathrm{M} \mathrm{NaCl} ; 0.3 \mathrm{M}$ trisodium citrate dihydrate); $5 \mathrm{mM}$ ethylene diamine tetra-acetic acid (EDTA) from $500 \mathrm{mM}$ stock $\mathrm{pH}$ 8.0; 0.5 volume deionised formamide; $100 \mu \mathrm{g} / \mathrm{mL}$ Heparin from 100 $\mathrm{mg} / \mathrm{mL}$ stock; $0.1 \%(\mathrm{v} / \mathrm{v})$ Tween-20; $1 \mathrm{X}$ Denhardt's from 100X stock $(2 \%(\mathrm{~m} / \mathrm{v})$ Ficoll type $400 ; 2 \%(\mathrm{w} / \mathrm{v})$ polyvinylpyrrolidone $\mathrm{K} 30 ; 2 \%(\mathrm{w} / \mathrm{v})$ bovine serum albumin); $100 \mu \mathrm{g} / \mathrm{mL}$ single-stranded salmon sperm DNA from $10 \mathrm{mg} / \mathrm{mL}$ stock.

4X wash: 0.2 volume $20 X$ SSC stock; 0.5 volume formamide; $0.1 \%(v / v)$ Tween-20.

2X wash: 0.1 volume 20X SSC stock; 0.5 volume formamide; $0.1 \%(\mathrm{v} / \mathrm{v})$ Tween-20.

1X wash: 0.05 volume 20X SSC stock; 0.5 volume formamide; $0.1 \%$ (v/v) Tween-20.

MAB (maleic acid buffer): $0.1 \mathrm{M}$ maleic acid from $1 \mathrm{M}$ stock $\mathrm{pH} 7.5 ; 0.15 \mathrm{M} \mathrm{NaCl}$ from 5 M stock.

Block solution: $2 \%(\mathrm{v} / \mathrm{v})$ block from $10 \%(\mathrm{w} / \mathrm{v})$ stock in MAB.

Antibody solution: anti-DIG fab fragments diluted 1:10,000 in block solution.

1X APTw (Alkaline Phosphatase buffer + Tween 20): 0.2 volume 5X AP buffer stock (0.5 M Tris pH 9.5 from $1 \mathrm{M}$ stock; $0.5 \mathrm{M} \mathrm{NaCl}$ from $5 \mathrm{M}$ stock); $0.1 \%$ (v/v) Tween-20. 
Colour detection buffer: $1 \mathrm{X}$ APTw; $50 \mathrm{mM} \mathrm{MgCl}$ from 1 M stock; $450 \mu \mathrm{g} / \mathrm{mL} \mathrm{NBT}$ from $100 \mathrm{mg} / \mathrm{mL}$ stock in DMF; $175 \mu \mathrm{g} / \mathrm{mL}$ BCIP from $50 \mathrm{mg} / \mathrm{mL}$ stock in water.

Stop solution: $0.1 \mathrm{M}$ glycine $\mathrm{pH} 2.2$ from $1 \mathrm{M}$ stock; $0.1 \%$ (v/v) Tween-20.

PFA: 4\% (v/v) PFA in 1x PBTw.

$60 \%$ glycerol: $60 \%$ glycerol $(\mathrm{v} / \mathrm{v})$ in water.

\section{Results and discussion}

A previously described WMISH protocol for molluscan embryos and larvae $[15,16]$, did not yield consistent or satisfactory WMISH signals in L. stagnalis so we focused on a few key steps of sample preparation we believed to cause background, weak WMISH signals and non-specific staining. A summary of the treatments we found to generate the clearest WMISH signals for each developmental stage (a "protocol at a glance") is attached at the end of this chapter. The results of control experiments using no antibody and no riboprobe (which generated no signals) are provided in Figure 7.

\section{Removal of the intra-capsular fluid}

The fluid that bathes L. stagnalis larvae during their encapsulated development is characterised by a high viscosity and adheres to the embryo following decapsulation. An incubation step with the mucolytic reagent NAC apparently leads to a superior preservation of the overall morphology (Figs. 1-4), possibly by removing the intracapsular fluid and allowing for better penetration of the fixative. However, for two of the three genes studied ( $\beta$-tubulin and engrailed) a treatment with NAC prior to fixation resulted in a significant reduction of both signal intensity and non-specific background staining, especially in embryos between two and three dpfc (Figs. 1 and $2, \mathrm{C}, \mathrm{D}, \mathrm{G}$ and $\mathrm{H}$ ). A specific example of this can be seen in $\beta$-tubulin ${ }^{+}$cells surrounding the shell gland and in the posterior region of the developing foot, which cannot be found in NAC-treated specimens (Fig. 4B and D $c f .4 \mathrm{~F}$ and $\mathrm{H}$, black arrows). Older larvae also display reduced signal intensity if treated with NAC (Figs. 1 and 2, K and $0)$. However, when NAC treatment was combined with a reduction step this effect was apparently reversed (Figs. 1 and 2, L and P). In three to six dpfc larvae for $\beta$-tubulin (Fig. 1L), and in two to three dpfc larvae for COE (Fig. 3H), this $\mathrm{NAC}+$ reduction treatment gave the best signal to background ratio. In contrast to 
$\beta$-tubulin and COE, in both two to three dpfc and three to six dpfc larvae, omitting the NAC treatment and treating with the appropriate reduction solution generated the highest signal to noise ratio for engrailed (Fig. $2 \mathrm{~F}$ and $\mathrm{N}$ ).

Our overall impression of treating L. stagnalis larvae with NAC is most beneficial when working with probes, which tend to generate non-specific background when combined with a reduction step. The optimal NAC-treatment depends on the ontogenetic stage treated - excessive treatment with NAC leads to compromised morphology of younger larvae.

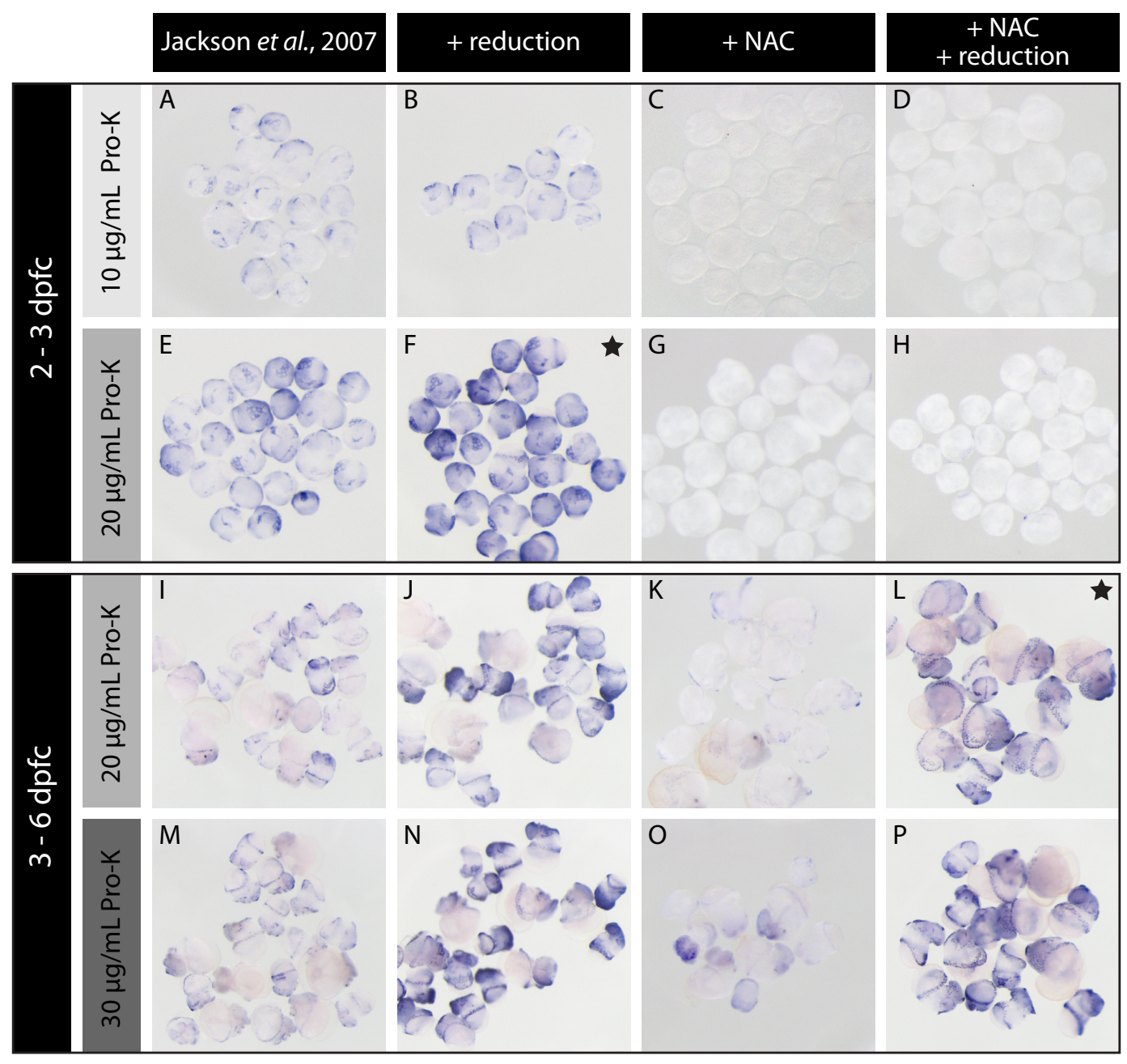

Fig. 1. Overview of $\beta$-tubulin WMISH signals produced by different experimental treatments. L. stagnalis larvae of different ages were subjected to a WMISH protocol similar to that described by Jackson et al. [16] (A, E, I and M). This protocol was then modified by the addition of a reduction treatment (B, F, J, and N), a NAC treatment (C, G, K and O) or a NAC and reduction treatment (D, H, L and $\mathrm{P}$ ) and then hybridised with an anti-sense probe to $\beta$-tubulin. In larvae of all ages a reduction treatment improves WMISH signal intensity. Treatment with NAC significantly reduces the signal strength in younger larvae (C, D, G and $\mathrm{H}$ ), but yields the optimal signal to background ratio in older larvae when combined with a reduction treatment ( $\mathrm{L}$ and P). Optimal treatments (F and L) are indicated by black stars. 


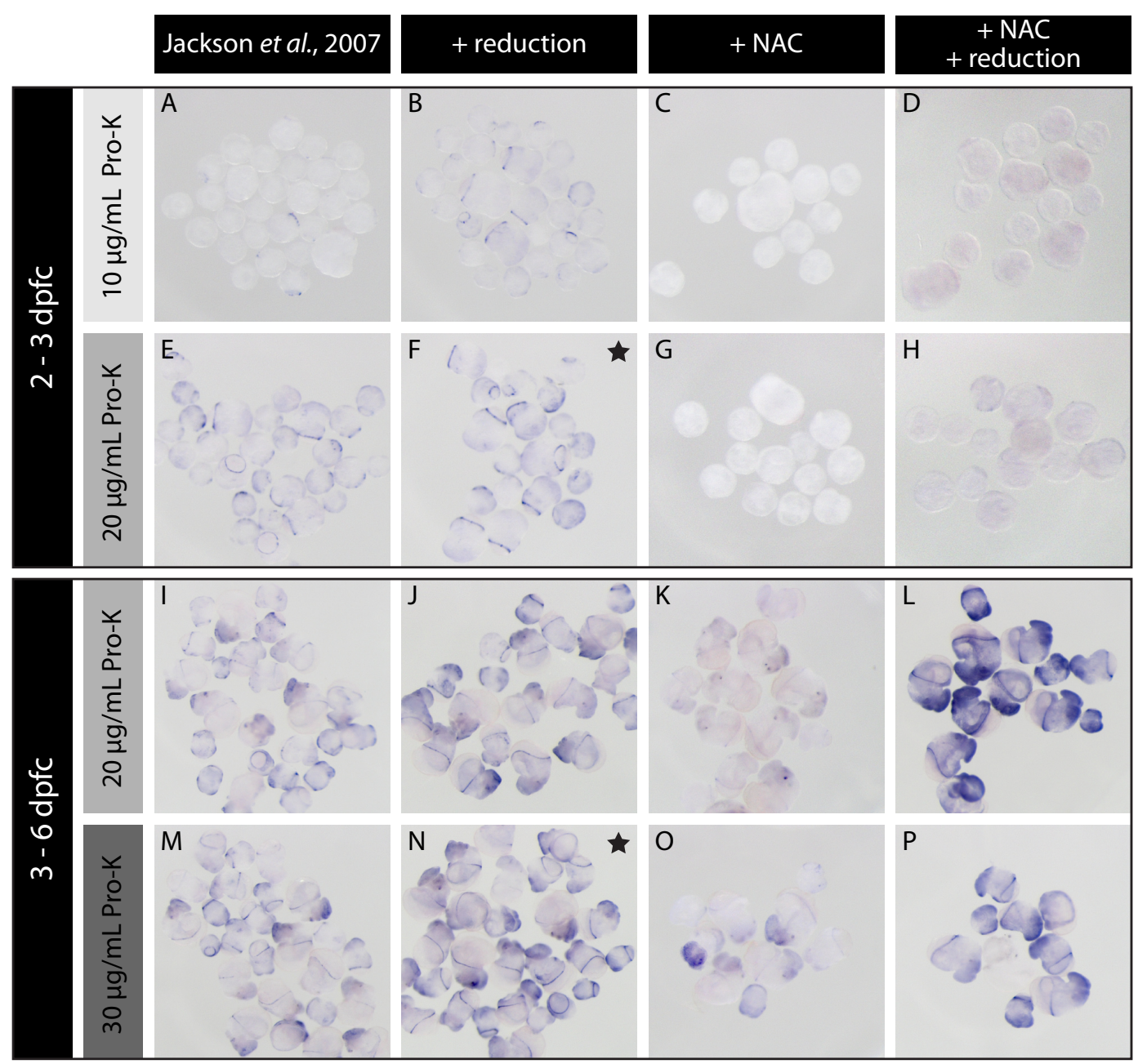

Fig. 2. Overview of engrailed WMISH signals produced by different experimental treatments. L. stagnalis larvae of different ages were subjected to a WMISH protocol similar to that described by Jackson et al. [16] (A, E, I and M). This protocol was then modified by the addition of a reduction treatment (B, F, J, and N), a NAC treatment (C, G, K and O) or a NAC and reduction treatment (D, H, L and $\mathrm{P}$ ) and then hybridised with an anti-sense probe to engrailed. In larvae of all ages a reduction treatment improves WMISH signal intensity. In larvae between three and six dpfc (I-P), the reduction treatment can even partially recover the WMISH signal, which is diminished by the NAC treatment (G $c f$. $\mathrm{H}$ and $\mathrm{K}$ and $\mathrm{O} c f . \mathrm{L}$ and $\mathrm{P}$ ). Optimal treatments ( $\mathrm{F}$ and $\mathrm{N}$ ) are indicated by black stars.

\section{Permeabilisation by treatment with DTT and detergents}

A permeabilisation step following fixation using a solution containing DTT and the detergents sodium dodecyl sulfate and NP-40 greatly increased the signal intensity for all investigated genes and developmental stages (Figs. 1-3). However this was at the cost of all material becoming highly fragile until dehydrated in ethanol. Both the incubation temperature and the concentration of this combined reduction/detergent treatment were adjusted for the two developmental time windows investigated (see Methods). Both specific and non-specific staining intensity is increased in 
reduced specimens. This can be compensated for by a reduction in the concentration of the applied riboprobe concentration (Fig. 4).

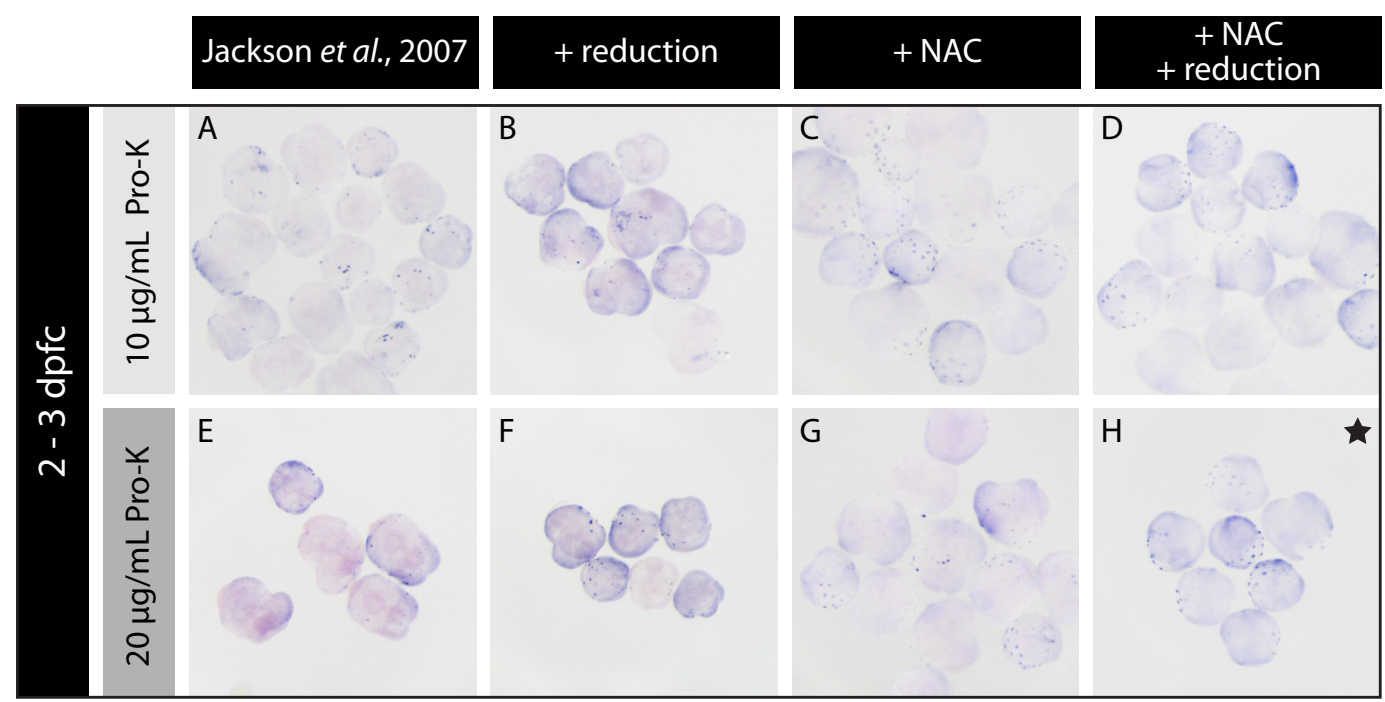

Fig. 3. Overview of $C O E$ WMISH signals produced by different experimental treatments.

L. stagnalis larvae of different ages were subjected to a WMISH protocol similar to that described by Jackson et al. [16] (A and E). This protocol was then modified by the addition of a reduction treatment (B and F), a NAC treatment (C and G) or a NAC and reduction treatment (D and H) and then hybridised with an anti-sense probe to $C O E$, which is only expressed in larvae younger than $3 \mathrm{dpfc}$. In contrast to $\beta$-tubulin and engrailed, the best COE WMISH signal was not generated by a sole reduction treatment. All NAC-treated samples hybridised with an anti-sense COE probe displayed more consistent and superior signal to noise ratios than those without a NAC treatment ( $C, D, G$ and $H$ ). The optimal treatment $(\mathrm{H})$ is indicated by a black star.

\section{Enzymatic permeabilisation with Pro-K}

The optimal Pro-K concentration for tissue permeabilisation and mRNA target unmasking depends on the incubation temperature, incubation time and the ontogenetic stage of the material. In general, under-treatment yields weak WMISH signals while over-treatment increases background staining and leads to compromised tissue morphology. We tested a wide range of Pro-K concentrations in combination with various NAC and reduction regimes. Preliminary experiments revealed that treatment with proteinase- $\mathrm{K}$ at concentrations of $0-2 \mu \mathrm{g} / \mathrm{mL}$ drastically reduced signal intensity while treatment at concentrations of 40-50 $\mu \mathrm{g} / \mathrm{mL}$ compromised tissue integrity (data not shown). We therefore used concentrations of 10 and $20 \mu \mathrm{g} / \mathrm{mL}$ for two to three dpfc old larvae, and 20 and 30 $\mu \mathrm{g} / \mathrm{mL}$ for larvae between three and six dpfc in the final overall comparison. For larvae between two and three dpfc we found the higher concentration of Pro-K to be optimal (Figs. 1-3). In larvae between three and six dpfc the best signal to noise ratio 
was achieved with $20 \mu \mathrm{g} / \mathrm{mL}$ for $\beta$-tubulin (Fig. 1L) and $30 \mu \mathrm{g} / \mathrm{mL}$ for engrailed (Fig. $2 \mathrm{~N}$ ). COE is apparently not expressed in larvae between three and six dpfc.

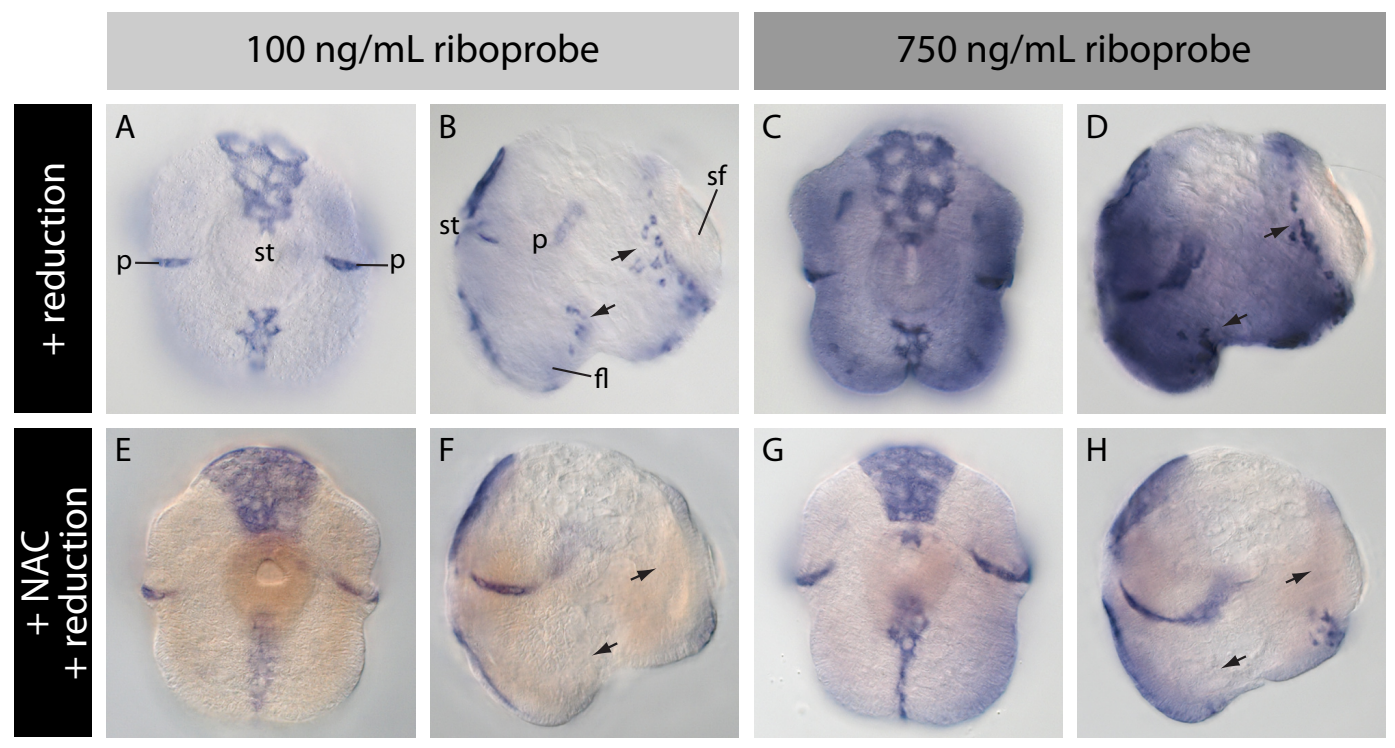

Fig. 4. Treatment with NAC decreases both signal and non-specific background intensity. Treatment with NAC conceals $\beta$-tubulin expression domains present in non-NAC treated larvae (B $c f$. $\mathrm{F}$ and $\mathrm{D} c f . \mathrm{H}$, black arrows). NAC treatment also visibly reduces non-specific background staining, however optimizing the amount of riboprobe can compensate for such non-specific background staining in non-NAC treated samples (A and B $c f$. C and D). A, E, C and G are ventral/anterior views of three dpfc old larvae. B, F, D and $\mathrm{H}$ are lateral views. Panels D and F are reflected about the vertical axis for clarity of presentation. Positions of the stomodeum (st), shell field (sf), foot lobe (fl) and prototroch (p) are indicated.

Removal of non-specific background with triethanolamine + acetic anhydride (TEAAA) In preliminary experiments using a wide variety of riboprobes to different genes we obtained a strong, well-defined WMISH signal located at the periphery of the shell field and in the radular sac (Fig. 5, black arrows). To determine whether these staining patterns represented genuine probe/target hybridisation events, or nonspecific binding of the probe, we treated fixed larval material with RNAse-A prior to hybridisation. As a control, all expected $\beta$-tubulin staining was abolished following this RNAse treatment, confirming the degradation of all target mRNA as expected (Fig. 5E). In RNAse treated embryos hybridised with probes that generated the suspicious shell field and radula patterns this signal was still present (Fig. 5F-H), indicating these WMISH patterns represent high affinity binding of the riboprobe to non-target molecules. In order to address this background staining, we assessed the effect of a triethanolamine + acetic anhydride (TEAAA) treatment. Treatment of biological substrates with TEAAA is a common practice for many WMISH protocols, 
and decreases non-specific binding of labelled probes through the acetylation of polar and charged groups [17]. For L. stagnalis treatment with TEAAA following Pro-K digestion successfully abolished the non-specific WMISH signal in the larval shell and in the radular sac (Fig. 5J-L). This TEAAA treatment is therefore critical for the correct interpretation of genes with expression patterns associated with the shell gland and shell field, engrailed is a good example of this (Fig. 6I-P).

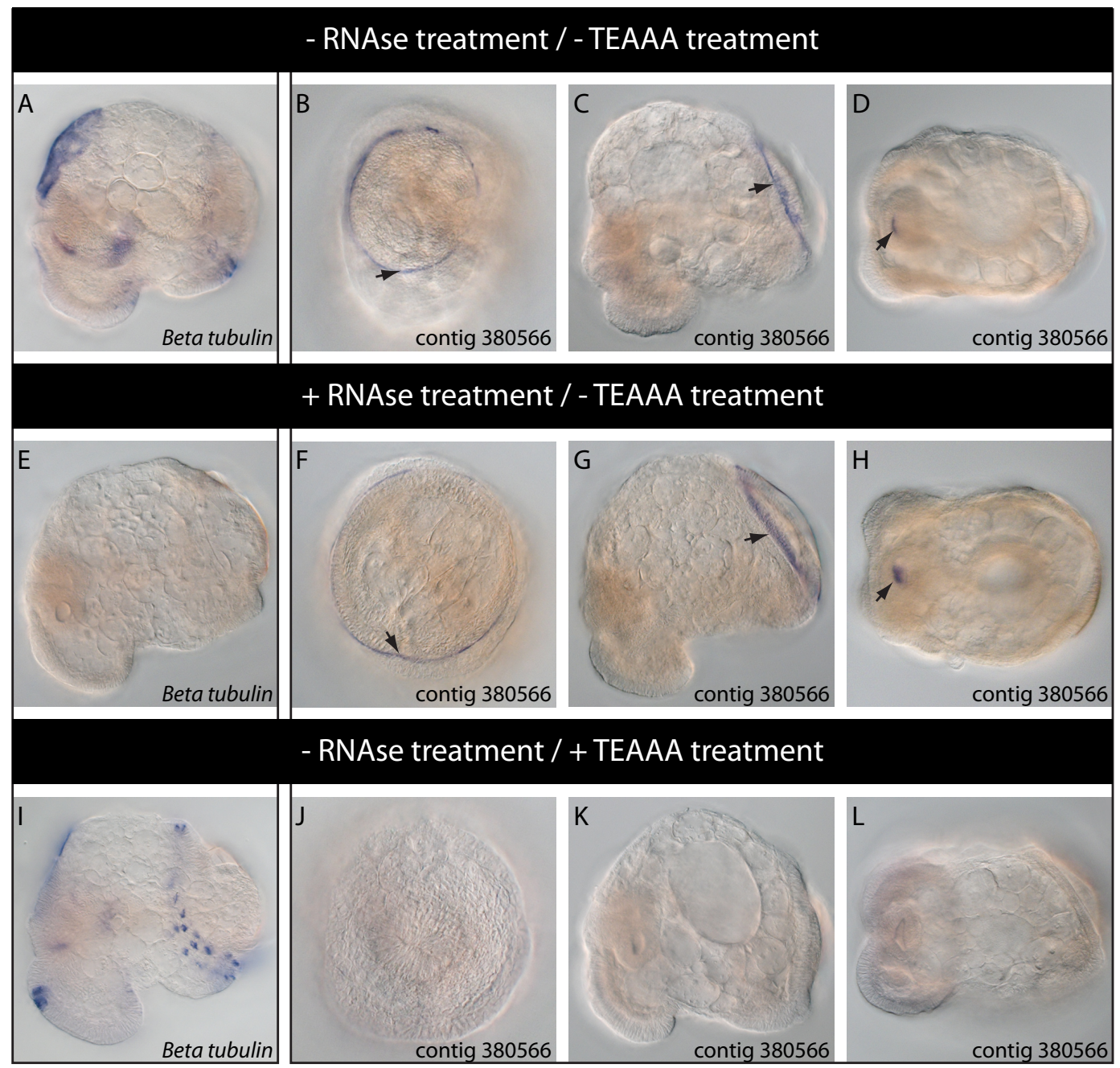

Fig. 5. Non-specific probe binding is eliminated by treatment with TEA and acetic anhydride. A well defined and consistent WMISH stain is observed for a variety of probes in the periphery of the shell field (arrows in B and C) and in the radular sac (arrow in D). These patterns remain following a pre-hybridisation treatment with RNAse $(\mathrm{F}-\mathrm{H})$ indicating that they are the result of non-specific probe binding, in case of the shell field stain most likely to secreted insoluble shell-associated material. Treatment with TEAAA abolishes this non-specific stain (J-L). B, F and J are dorsal views of larvae between three and four dpfc. C, G and K are lateral views of larvae of the same age with the shell gland oriented to the right. D, H and L are ventral views of larvae of the same age. Panels C, G and $\mathrm{K}$ are reflected about the vertical axis for clarity of presentation. 


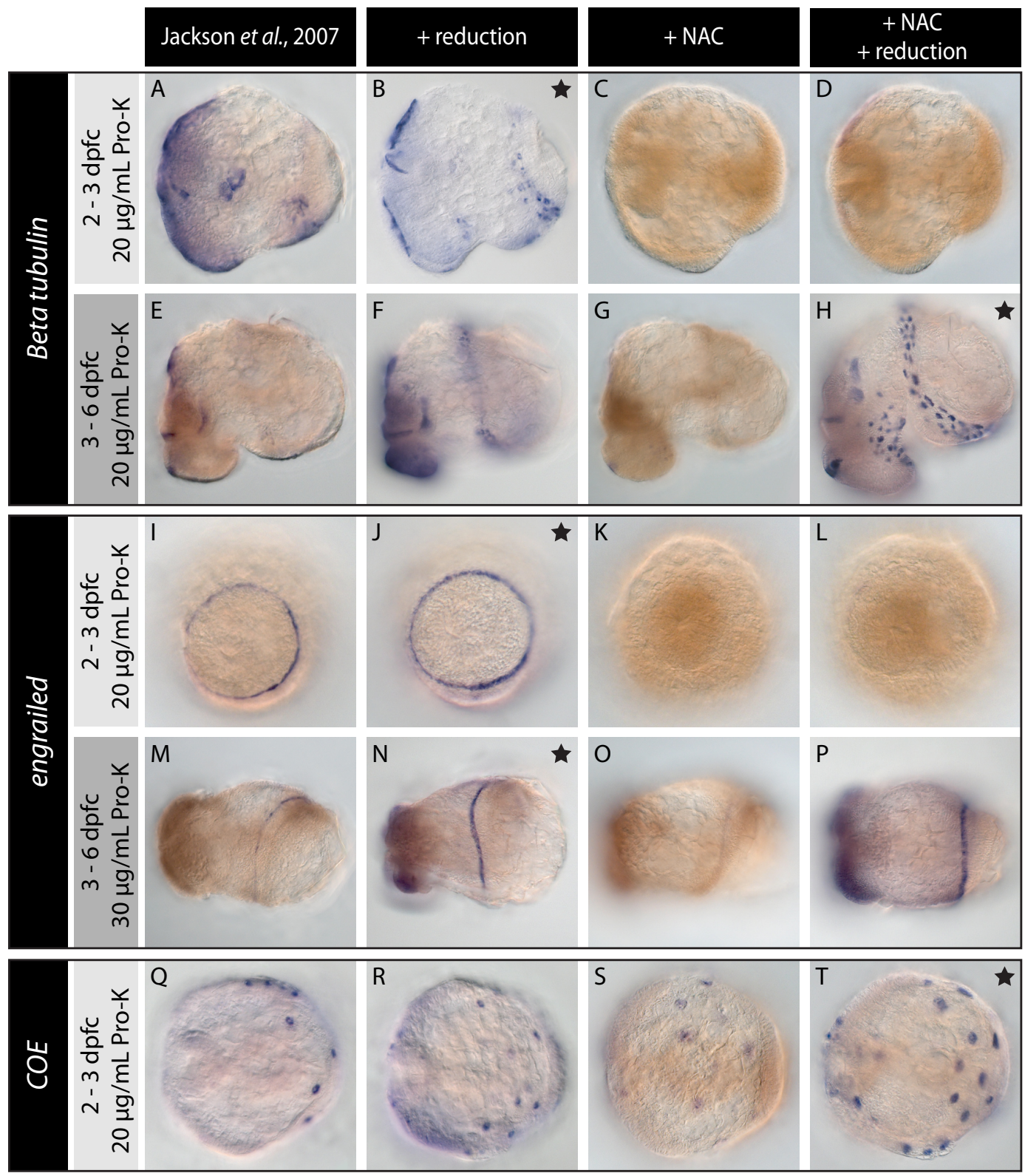

Fig. 6. The optimal sample preparation regime for WMISH varies with respect to the target gene and the developmental stage. A to $\mathrm{H}$ show larvae from a lateral perspective with the shell gland or shell oriented to the right. I to $\mathrm{P}$ are a dorsal views. $\mathrm{Q}$ to $\mathrm{T}$ are apical views. $\mathrm{M}$ to $\mathrm{P}$ have been treated with $30 \mu \mathrm{g} / \mathrm{mL}$ Pro-K, all other samples at $20 \mu \mathrm{g} / \mathrm{mL}$. The optimal treatment for each gene and developmental stage is indicated by a black star. 
The effect of the storing fixed material in Ethanol vs. Methanol

Methanol is used to dehydrate and store fixed embryonic and larval material at low temperatures $\left(-20^{\circ} \mathrm{C}\right)$ in many WMISH protocols. Due to the relatively high toxicity of $\mathrm{MeOH}$ relative to $\mathrm{EtOH}$ we assessed the effect of storing fixed L. stagnalis larvae in $\mathrm{MeOH}$ or EtOH on the WMISH signal generated by $\beta$-tubulin, engrailed and COE. We found no consistent or significant difference with respect to any of the signals generated (data not shown).

Table 1. Primer sequences used to isolate gene fragments for riboprobe syntheses.

\begin{tabular}{ccc}
\hline Gene & $\begin{array}{c}\text { sequence of forward primer } \\
\text { (5' to 3') }\end{array}$ & $\begin{array}{c}\text { sequence of reverse primer } \\
\text { (5' to 3') }\end{array}$ \\
\hline$\beta$-tubulin & TGTGGAATGGATCCCCAACAATGTCA & TCACTCAGGAGCTTTGATACGGCTTG \\
\hline engrailed & CGTCTCCGTCTCCGCCTCTCA & GCCCGAGCTCATCCGCCAGGTTCTTCC \\
\hline COE & TGTATCTCACCACCTCGTCACATC & CCGCCAGCTGACTCGGGTAGGCGTTG \\
\hline
\end{tabular}

Probe and target influence the optimal WMISH protocol for a given ontogenetic stage An ideal WMISH result represents a balance between a clear and consistent signal on the one hand, and the preservation of morphology and minimal background staining on the other. When systematically compared, the general effects of treatment with NAC, reduction and different Pro-K concentrations on the WMISH signals we investigated are in congruence. Our results show that for all three genes signal intensity and reproducibility markedly benefit from a reduction treatment (Figs. 1-3). Tissue integrity is slightly better preserved and non-specific background is minimized if samples are treated with NAC prior to fixation, but this may come at the cost of a reduction in signal strength. It should be noted that the best results for each of the three genes we investigated are not achieved with the same NAC treatment; most notably two to three dpfc larvae require NAC treatment when hybridised with a COE probe, but do not require NAC treatment when hybridised with $\beta$-tubulin or engrailed probes (Figs. $1 \mathrm{~F}, 2 \mathrm{~F}, 3 \mathrm{H}$ and $6 \mathrm{~B}, \mathrm{~J}$ and $\mathrm{T}$ ). In addition, the optimal Pro-K treatment for a given ontogenetic stage depends on the gene being investigated; for example three to six dpfc larvae are optimally treated with $20 \mu \mathrm{g} / \mathrm{mL}$ Pro-K for $\beta$-tubulin, and with $30 \mu \mathrm{g} / \mathrm{mL}$ for engrailed (Fig. $1 \mathrm{~L} c f$. Fig. $2 \mathrm{~N}$ ). 

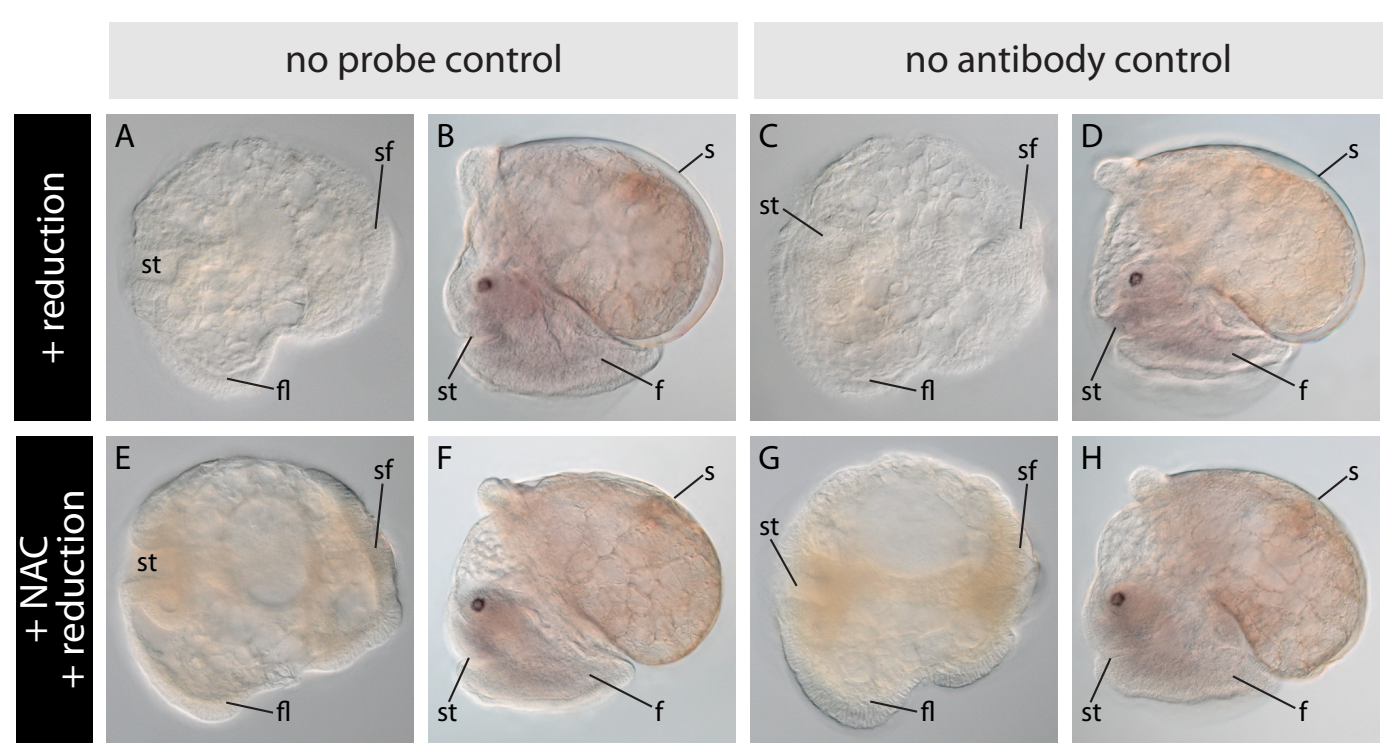

Fig. 7. Control experiments for the optimised sample preparations. Control WMISH experiments lacking riboprobe $(\mathrm{A}, \mathrm{B}, \mathrm{E}$ and $\mathrm{F}$ ) or antibody $(\mathrm{C}, \mathrm{D}, \mathrm{G}$ and $\mathrm{H})$ demonstrate the absence of any nonspecific colour reaction for reduced samples (A-D) or reduced+NAC treated samples (E-H) for larvae between two and three $\operatorname{dpfc}(\mathrm{A}, \mathrm{C}, \mathrm{E}$ and $\mathrm{G})$ and three to six dpfc (B, D, F and G). All embryos are shown from a lateral perspective. Indicated are the positions of the eye (ey), the stomodeum (st), the developing mantle margin ( $\mathrm{mm}$ ), the shell field (sf) or shell (s) and the foot lobe (fl) or foot (f). Panels $A$ and $E$ are reflected about the vertical axis for clarity of presentation.

\section{Conclusions}

Our study highlights the complex interactions that exist between probe, target and the optimal set of substrate preparation steps for each ontogenetic stage of $L$. stagnalis that will yield the best possible WMISH signal. These results indicate that within a broad window of recommended practices (always including a reduction treatment, treatment of two to three dpfc larvae with $10-20 \mu \mathrm{g} / \mathrm{mL}$ Pro-K and three to six dpfc larvae with $20-30 \mu \mathrm{g} / \mathrm{mL}$, always include treatment with TEAAA) each probe and target will need to be empirically optimised regarding NAC treatment and probe parameters (concentration, length and sequence composition). We also demonstrate that non-specific shell field and radula staining can easily be abolished with a TEAAA treatment. We hope this WMISH protocol will serve to raise the profile and popularity of $L$. stagnalis as a tractable experimental model for molluscan evo-devo research, a niche that is currently under-populated. 


\section{Acknowledgements}

We gratefully acknowledge Luciana Macis for the maintenance of snail lines. We also acknowledge Diana Bauermeister for isolating engrailed and $\beta$-tubulin sequences used for riboprobe synthesis. This work was supported by Deutsche Forschungsgemeinschaft (DFG) funding to DJJ through the CRC Geobiology and the German Excellence Initiative, and DFG project number JA 2108/1-1.

\section{References}

1. Christodoulou F, Raible F, Tomer R, Simakov O, Trachana K, Klaus S, Snyman H, Hannon GJ, Bork P, Arendt D: Ancient animal microRNAs and the evolution of tissue identity. Nature 2010, 263:1084-1088.

2. $\quad$ Arendt D: The evolution of cell types in animals: emerging principles from molecular studies. Nat Rev Gen 2008, 9:868-882.

3. Hashimoto N, Kurita Y, Wada H: Developmental role of dpp in the gastropod shell plate and co-option of the $d p p$ signaling pathway in the evolution of the operculum. Dev Biol 2012, 366:367-373.

4. Raven CP: Morphogenesis in Limnaea stagnalis and its disturbance by lithium. J Exp Zool 1952, 121:1-77.

5. Timmermans LPM: Studies on shell formation in molluscs. Neth J Zool 1969, 19:63-78.

6. Kniprath E: Zur Ontogenese des Schalenfeldes von Lymnaea stagnalis. Dev Gen Evol 1977, 181:11-30.

7. Liu MM, Davey JW, Banerjee R, Han J, Yang F, Aboobaker A, Blaxter ML, Davison A: Fine Mapping of the Pond Snail Left-Right Asymmetry (Chirality) Locus Using RAD-Seq and Fibre-FISH. PLOS ONE 2013, 8:e71067.

8. Hohagen J, Jackson DJ: An ancient process in a modern mollusc: early development of the shell in Lymnaea stagnalis. BMC Dev Biol 2013, 13:27.

9. Voronezhskaya E: Apical sensory neurones mediate developmental retardation induced by conspecific environmental stimuli in freshwater pulmonate snails. Development 2004, 131:3671-3680. 
10. Raven CP: The Development of the Egg of Limnaea Stagnalis L. From the First Cleavage Till the Trochophore Stage, With Special Reference To Its" Chemical Embryology". Arch. Neer. Zool. 1946, 7:353-434.

11. Elbers PF, Bluemink JG: Pinocytosis in the developing egg of Limnaea stagnalis L. Exp Cell Res 1960, 21:619-622.

12. Morrill JB: Development of the Pulmonate Gastropod, Lymnaea. In Developmental Biology of Freshwater Invertebrates Edited by Harrison FW, Cowden RR. New York: Alan R. Liss, Inc.; 1982:399-483.

13. Bayne CJ: Histochemical studies on the egg capsules of eight gastropod molluscs. J Moll Stud 1968, 38:199-212.

14. Pearson BJ, Eisenhoffer GT, Gurley KA, Rink JC, Miller DE, Sánchez Alvarado A: Formaldehyde-based whole-mount in situ hybridisation method for planarians. Dev Dyn 2009, 238:443-450.

15. Jackson D, McDougall C, Green K, Simpson F, Worheide G, Degnan B: A rapidly evolving secretome builds and patterns a sea shell. $B M C B i o l$ 2006, 4:40.

16. Jackson DJ, Wörheide G, Degnan BM: Dynamic expression of ancient and novel molluscan shell genes during ecological transitions. BMC Evol Biol 2007, 7:160.

17. Zeller R, Rogers M, Haramis AG, Carrasceo AE: In Situ Hybridisation to Cellular RNA. Curr Protoc Mol Biol 2001, 14.3.1-14.3.16

\section{Credits}

I carried almost all experimental procedures. I participated in designing this study and drafting the manuscript and contributed some of the aspects presented in the discussion.

Ines Herlitze assisted with the RNAse and TEAAA experiments and participated in the design of these experiments.

Daniel J. Jackson conceived and supervised the study. He participated in designing the study and drafting the manuscript and contributed most of the ideas stated in the introduction and some of the discussed aspects. 


\section{WMISH "protocol at a glance" for early larval stages of Lymnaea stagnalis}

We perform all sample preparation steps (Day 1) in 6 well tissue culture tissues using $3 \mathrm{~mL}$ of each solution. Where indicated, gentle agitation (150 rpm) on a flatbed mixer encourages the larvae to migrate to the center of the well making aspiration of the liquid from the edge of the well under a microscope easier. This also allows for the removal of any physically damaged material. All steps are carried out at room temperature unless otherwise indicated.

\section{Day 1: NAC, fixation, reduction and dehydration:}

1. Free capsules from jelly string and larvae from their capsules.

2. Optional (dependent on target gene): Treat larvae between 2 and $3 \mathrm{dpfc}$ for 5 min with 2.5\% NAC in PBS and larvae between 3 and 6 dpfc $2 \mathrm{X}$ each 5 min with 5\% NAC in PBS.

3. Transfer larvae into 4\% PFA in PBS and incubate without agitation for 5 min. After 5 min begin gentle agitation.

4. Remove fixative and wash larvae for $5 \mathrm{~min}$ in PBTw with gentle agitation.

5. Treat larvae between 2 and $3 \mathrm{dpfc}$ in $0.1 \mathrm{X}$ reduction solution for $10 \mathrm{~min}$ at room temperature and larvae between 3 and $6 \mathrm{dpfc}$ in pre-warmed $1 \mathrm{X}$ reduction solution at $37^{\circ} \mathrm{C}$. Do not agitate at this step. Note: samples are highly fragile in this solution until complete dehydration and must be handled with care.

6. Rinse with one exchange of PBTw without agitation.

7. Exchange for $50 \% \mathrm{EtOH}$ and let sit for 5-10 min without agitation.

8. Exchange for $100 \% \mathrm{EtOH}$ and let sit for 5-10 min with gentle agitation.

9. Replace with fresh $100 \% \mathrm{EtOH}$ and store at $-20^{\circ} \mathrm{C}$.

\section{Day 2: Rehydration, Pro-K, TEA, pre-hybridisation and hybridisation:}

10. Replace $100 \%$ EtOH with $50 \% \mathrm{EtOH}$ and gently agitate for 5-10 min.

11. Exchange for PBTw and gently agitate for 5-10 min. Repeat twice.

12. Incubate larvae between 2 and $3 \mathrm{dpfc}$ in $20 \mu \mathrm{g} / \mathrm{mL}$ proteinase $\mathrm{K}$ in PBTw for $10 \mathrm{~min}$ at room temperature. Incubate larvae between 3 and $6 \mathrm{dpfc}$ with $30 \mu \mathrm{g} / \mathrm{mL}$ proteinase $\mathrm{K}$ for $10 \mathrm{~min}$ at room temperature. Do not agitate at this step.

13. Replace Pro-K solution with $2 \mathrm{mg} / \mathrm{mL}$ Glycine and incubate for $5 \mathrm{~min}$ without agitation. 
14. Exchange for fresh glycine and incubate for $5 \mathrm{~min}$ without agitation.

15. Rinse with PBTw. Repeat twice without agitation.

16. Replace with $1 \%$ TEA and let sit for 5 min without agitation.

17. Replace with fresh $1 \%$ TEA and incubate for 5 min without agitation.

18. Exchange for TEAAA and incubate for 5 min without agitation. Note: prepare TEAAA immediately before use.

19. Exchange for fresh TEAAA and incubate for a further $5 \mathrm{~min}$ without agitation.

20. Wash three times with PBTw for 5-10 min.

21. Postfix in 4\% PFA in PBTw for 10 min.

22. Wash three times with PBTw for 5-10 min.

23. Warm hybridisation buffer to room temperature.

24. Replace PBTw with pre-warmed hybridisation buffer and incubate for $15 \mathrm{~min}$ at room temperature.

25. Heat all samples to hybridisation temperature.

26. Exchange hybridisation buffer once and incubate for two hours at hybridisation temperature.

27. Dilute riboprobes in hybridisation buffer at room temperature.

28. Denature riboprobe dilutions for $10 \mathrm{~min}$ at $75^{\circ} \mathrm{C}$.

29. Replace hybridisation buffer with riboprobe dilutions.

30. Incubate overnight at hybridisation temperature.

\section{Day 3: Hot washes, antibody block and antibody incubation:}

31. Pre-warm all wash solutions to hybridisation temperature.

32. Wash all samples three times in $4 \mathrm{X}$ wash buffer for 15 min each at hybridisation temperature.

33. Wash all samples three times in $2 \mathrm{X}$ wash buffer for 15 min each at hybridisation temperature.

34. Wash all samples three times in $1 \mathrm{X}$ wash buffer for 15 min each at hybridisation temperature.

35 . Wash all samples once with $1 \mathrm{X}$ SSC $+0.1 \%$ Tween at hybridisation temperature.

36. Allow samples to cool to room temperature.

37. Wash twice in $1 \mathrm{X}$ SSC $+0.1 \%$ Tween for each $15 \mathrm{~min}$.

38. Replace with $\mathrm{MAB}$ and let sit for $10 \mathrm{~min}$. Repeat.

39. Cool samples to $10^{\circ} \mathrm{C}$. 
40. Pre-cool block solution.

41. Replace with cold block solution and incubate for $1.5 \mathrm{~h}$.

42. Exchange for fresh cold block solution and incubate for $1.5 \mathrm{~h}$.

43. Replace with cold antibody solution and incubate for several hours.

44. Exchange for fresh cold antibody solution and incubate overnight.

\section{Day 4: Antibody washes and colour development:}

45. Wash 10 times with PBTw for 10 min each at $10^{\circ} \mathrm{C}$.

46. Wash 5 times with PBS for 10 min each at $10^{\circ} \mathrm{C}$.

47. Allow samples to warm to room temperature.

48. Replace with 1X APTw and incubate for $10 \mathrm{~min}$.

49. Exchange for fresh $1 \mathrm{X}$ APTw and incubate for $10 \mathrm{~min}$.

50. Replace with colour detection buffer and monitor until the signal to background ratio is optimal.

51. Stop the colour reaction by one wash with $0.1 \mathrm{M}$ Glycine $\mathrm{pH} 2$ for $5 \mathrm{~min}$.

52. Rinse samples in PBTw.

53. Postfix samples in $4 \% \mathrm{PFA}$ in $\mathrm{PBTw}$ for $2 \mathrm{~h}$ at room temperature or at $4^{\circ} \mathrm{C}$ over night.

54. Wash twice with PBTw for each 10 min.

55. Wash twice in pre-warmed to $37^{\circ} \mathrm{C}$ deionized water and incubate for $10 \mathrm{~min}$ at $37^{\circ} \mathrm{C}$. Note: this step removes residual salts and detergent that might precipitate during the following dehydration.

56 . Samples can now either be mounted and cleared in $60 \%$ glycerol for photo documenting, or dehydrated and stored in $100 \% \mathrm{EtOH}$ at $-20^{\circ} \mathrm{C}$. Note: a dehydration in EtOH removes slight overall background staining. 


\section{Chapter 4}

Notch signalling during the early shell field development in Lymnaea stagnalis 


\section{Notch signalling during the early shell field development in Lymnaea stagnalis}

\section{Introduction}

The innovation of the molluscan shell more than 540 million years ago was of great importance for the generation of the various extant and extinct molluscan life forms. Nevertheless, surprisingly little is known about the molecular framework that underlies molluscan shell formation during development. The limited number of genes known to be associated with larval shell formation is wide of representing the gene regulatory network that governs this process $([1,2]$; see also pp. 11). In particular, they are expressed when the larval shell-secreting organ, the molluscan shell field, is already well differentiated. Hence, these genes are not likely to function in the initial specification of the larval shell field.

The developmental basis of molluscan shell field specification is poorly understood. However, the morphogenetic processes that precede the first shell field differentiation resemble a contact-mediated specification by induction event in disparate molluscan taxa $([3,4]$; reviewed in [5]). Briefly, future shell forming cells of the dorsal ectoderm establish a direct contact with endodermal cells of the underlying archenteron at the end of gastrulation. Upon this contact, the dorsal ectodermal cells assume a highly columnar shape, which is the first visible morphological differentiation of the shell field (see chapter 2, Fig. 2). Thus, the specification of the prospective shell field cells coincides with the forming contact between these cells and the underlying endodermal cells. If the molluscan shell field is truly induced by this contact, the genes that promote this process should operate in a contact-associated manner. Given the widespread occurrence of the contact event across molluscan classes (reviewed in [5]), these genes are further expected to have been in place at the time these lineages diverged. Strikingly few developmental signalling pathways are deployed in the numerous tightly regulated specification events during metazoan development: only seven evolutionarily conserved cell-cell signalling pathways exert control over the vast majority of early cell fate decisions [6].

Developmental gene expression studies in the early branching gastropod Haliotis asinina have revealed the expression of a delta-orthologue in shell forming cells before and after these cells differentiate into the larval shell field (Jackson, unpub- 
lished data). The delta gene encodes an intercellular signalling molecule that functions in the Notch pathway. This pathway exhibits many of the characteristics expected from the genetic subset that specifies the molluscan shell field. The present study evaluates the role of the Notch pathway during the early shell field development in the pulmonate gastropod Lymnaea stagnalis, in particular during the presumptively specifying contact event.

The Notch pathway is an evolutionary ancient intercellular signalling pathway and deployed repeatedly during the development of any metazoan. The core components of the canonical Notch pathway are conserved among the Metazoa while the origin of several molecular components even predates the divergence of the metazoan lineage [7]. During animal evolution, the Notch pathway has been coopted for diverse developmental processes such as pattern formation and neurogenesis. Herein, Notch signalling mainly regulates cellular identity and differentiation by two mechanisms: lateral inhibition and boundary formation which both can be combined with the asymmetric segregation of intrinsic pathway regulators (reviewed e.g. in [8-10]). During lateral inhibition, one cell signals to its neighbouring cells, which prevents them to adopt the same fate as the signalling cell [11]. During boundary formation, signalling occurs between two non-equivalent cell populations and induces the cells at their interface to adopt a third fate, which establishes a developmental boundary [9]. Lateral inhibition, for instance, occurs during the patterning of the neurogenic ectoderm in Drosophila (reviewed e.g. in [8]). Here, Notch signalling is involved in the selection of sensory neuron precursor cells from a field of equivalent cells with neural potential [12]. Initially, all cells of this proneural cluster express both the Notch ligand and the receptor as well as proneural genes of the achaete-scute complex. Small fluctuations in the expression of ligand and receptor are amplified by feedback mechanisms until all cells express either the ligand or the receptor. This determines the direction of Notch signalling events within the proneural cell cluster. The receptor expressing cells receive signals from their surrounding cells and transcriptionally activate Notch target genes of the hairy/enhancer of split complex. These genes in turn prevent the receptor-bearing cell to adopt the neural precursor fate and promote the fate of future epidermal cells. This divides the proneural clusters into cells expressing the Notch receptor, the hairy/enhancer of split genes and the proneural achaete-scute genes with an epidermal fate on the one hand and sensory neuron precursor cells 
expressing the Notch ligand and the proneural genes on the other hand [11]. Thus, lateral inhibition results in the transformation of an initially equivalent cell population into a field of different regularly spaced cells types.

Notch-mediated intercellular signalling is restricted to neighbouring cells: both the ligands and the receptors are membrane-bound proteins exposed by adjacent cells. Hence, Notch signalling requires direct cell-cell contact. The signal transduction mechanism is remarkably direct and does not involve secondary messengers (Fig. 1). The Notch receptor molecule functions in both receiving the extracellular signal and controlling the transcriptional activation of target genes in the nucleus.

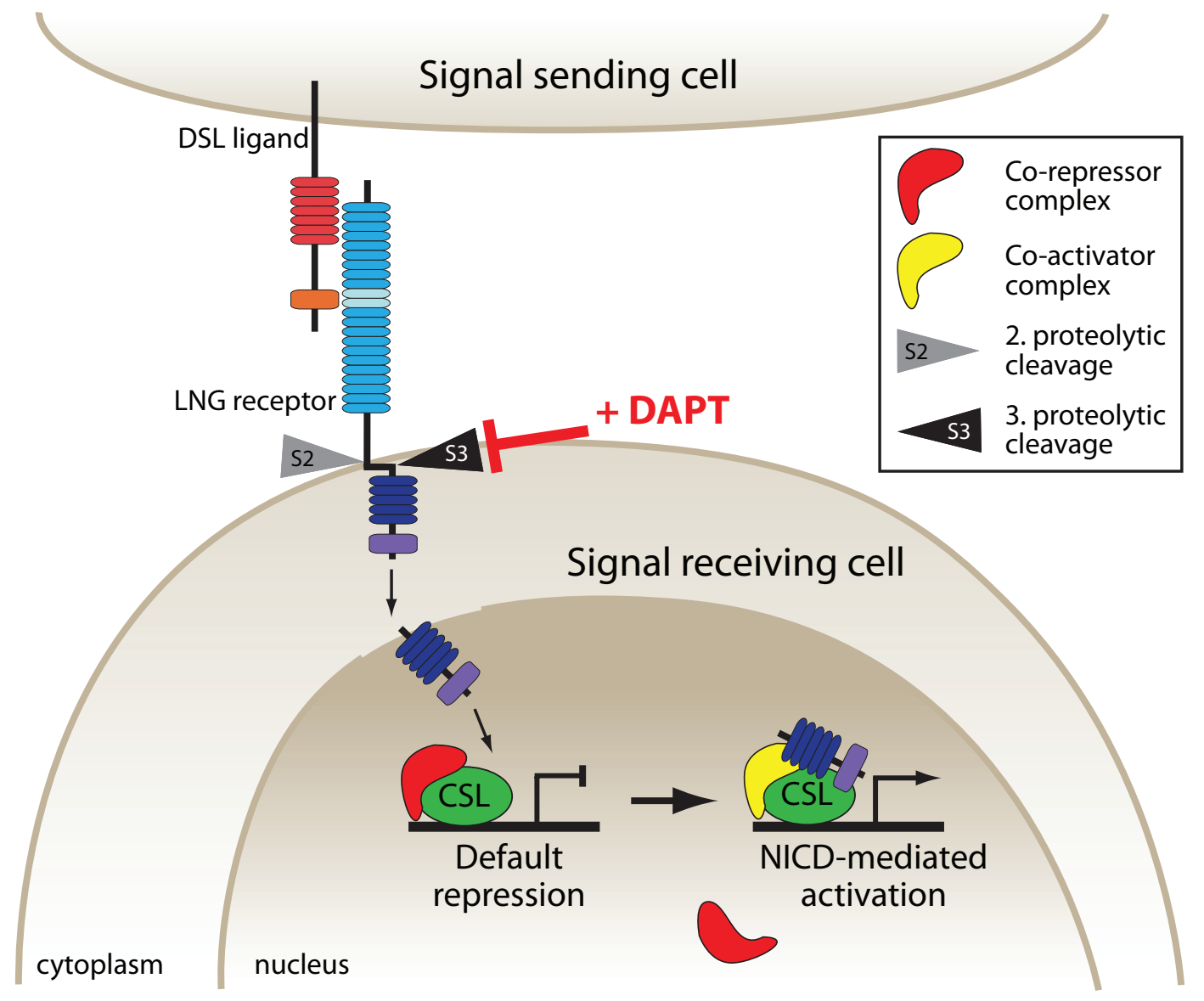

Fig. 1. Signal transduction in the canonical Notch pathway. The mechanism by which the pharmacological inhibitor DAPT prevents active signalling is depicted in red. 
The core components of the canonical Notch pathway are the DSL ligands (for Delta, Serrate and LAG-2, the known orthologues in fruit fly and nematode) and the Notch receptors (LNG proteins, for LIN-12, Notch and GLP-1). Both are type I transmembrane proteins that are composed of several conserved domains in a modular fashion and undergo extensive post-translational modifications before being exposed on the cell membrane in their mature form (reviewed in e.g. [13, 14]). The binding of a DSL ligand promotes two proteolytic cleavage events in the Notch receptor that results in the release of its intracellular fraction (called NICD) into the cytoplasm. The NICD then translocates to the nucleus and interacts with a DNAbound transcriptional repressor complex that includes the Notch effector CSL protein (for $\mathrm{CBF}-1, \mathrm{Su}(\mathrm{H}), \mathrm{LAG}-1$ ). Associated with CSL are co-repressors, which are displaced after binding of the NICD to the CSL protein. NICD and CSL assemble with co-activators into a transcriptional complex that activates target genes, in particular hairy/enhancer of split (hes) genes [15] (Fig. 1). The CSL protein therefore serves a dual function and can act as transcriptional repressor or activator depending on its interaction partners [6].

The Notch inhibitor DAPT (N-[N-(3,5-Difluorophenacetyl)-L-alanyl]-S-phenylglycine t-butyl ester) interferes with this intracellular signal transduction process. Precisely, DAPT prevents Notch signalling by inhibiting the gamma secretase complex that conducts the final proteolytic S3 cleavage causing the release of the NICD (Fig. 1).

I have studied the spatial expression of core components of the canonical Notch pathway to investigate the potential employment of Notch signalling during the shell field specification process in L. stagnalis. In particular, orthologues of the DSL ligands, the Notch receptors, the DNA binding CSL effector and downstream genes of the hairy/enhancer of split complex in L. stagnalis have been examined. Additionally, I have disturbed Notch signalling in vivo at the stage when the shell field specification is thought to take place, namely during the presumptively inducing contact event. The resulting phenotypes were assessed for the presence and appearance of the shell field using histochemical assays detecting endogenous enzyme activity. This study represents the first investigation specifically focusing on the molecular processes that underlie molluscan shell field specification. 


\section{Materials and Methods}

\section{Gene isolation}

RNA was extracted from different developmental stages and several adult tissues using TRI reagent (Ambion, \#AM9738) according to the manufacturer's instructions with an optional high salt precipitation step. Regular, 3' RACE and 5' RACE cDNA libraries were constructed from several adult tissues and developmental stages. For the regular cDNA synthesis, $1 \mu \mathrm{g}$ total RNA were combined with Oligo dT primer (20mer) at a final concentration of $2 \mu \mathrm{M}$ and water up to a total volume of $10 \mu \mathrm{L}$ and heated to $70^{\circ} \mathrm{C}$ for five minutes. The reactions were allowed to cool down to $42^{\circ} \mathrm{C}$ before $15 \mu \mathrm{L}$ reaction mix containing final $1 \mathrm{X}$ reverse transcription buffer, final $400 \mu \mathrm{M}$ dNTP and 200U MMLV-reverse transcriptase $\mathrm{H}^{-}$(Promega, \#M3682) were added. The synthesis was carried out at $42^{\circ} \mathrm{C}$ for 90 minutes and stopped by heating to $70^{\circ} \mathrm{C}$ for 15 minutes. The synthesis of $3^{\prime}$ RACE cDNA was essentially performed as described for regular cDNA but was primed with a modified Oligo dT primer that comprises an UPM adaptor at a final concentration of $0.4 \mu \mathrm{M}$. To generate 5' RACE cDNA, the reverse transcription was initiated using an Oligo dT primer (25mer) with two non-thymidine residues at a final concentration of $0.4 \mu \mathrm{M}$. Following 60 to 90 minutes incubation at $42^{\circ} \mathrm{C}, 3.5 \mu \mathrm{L}$ mix containing final $0.4 \mu \mathrm{M} \mathrm{dCTP}$, final $40 \mu \mathrm{g} / \mathrm{mL}$ bovine serum albumin, final $2 \mathrm{mM}$ manganese chloride tetrahydrate and final $500 \mu \mathrm{M}$ chimera primer with a UPM binding site and five guanidine ribonucleotides were added. The reaction was incubated for further 15 minutes at $42^{\circ} \mathrm{C}$ and then stopped by heating to $70^{\circ} \mathrm{C}$ for 15 minutes. The resulting $5^{\prime}$ cDNA was purified using the Qiagen QIAquick Gel Extraction Kit with the Qiagen PB buffer and eluted into final $50 \mu \mathrm{L}$ 5' RACE cDNA.

Partial or full coding sequences of genes encoding for seventeen Notch pathway members have been isolated by degenerate, standard or RACE PCR. Corresponding primer sequences are provided in appendix 2. Initial fragments of one DSL gene (contig 64109), a CSL gene (contig 165301) and a fringe-like modulator (contig 15835) were obtained by degenerate PCR. The amino acid sequence of the isolated DSL domain was compared to a draft genomic database of L. stagnalis using the Basic Local Alignment Search Tool (BLAST), resulting in the identification of several genomic contigs with high sequence similarity. Gene-specific primers were designed to amplify internal fragments and to isolate the full length coding sequence by 
RACE-PCR. Further Blast searches against the genomic database of L. stagnalis and the genome of Biomphalaria glabrata using conserved domains were performed for every novel fragment until all recovered genomic contigs corresponded to already identified loci. Gene orthologies have been designated by searches against the GenBank non-redundant protein database using the BlastX algorithm and by characterisation of the isolated sequences using the online search tool for conserved protein domains InterProScan. The DSL and Von Willebrand factor C (VWC) domains and the Epidermal Growth Factor-like (EGF) and ankyrin repeats were designated according to the smart algorithm. The MNNL, fringe, basic Helix Loop Helix (bHLH), Orange and all remaining Notch domains were determined using the Pfam algorithm. To detect transmembrane regions in the LNG and DSL transcripts the online search tool TMHMM Server v. 2.0 was used. To visualise the domain compositions of isolated genes, MyDomains image creator of Prosite was used.

\section{Whole mount in situ hybridisation}

Antisense riboprobes were synthesised for each gene as described in chapter 3. For the DSL contig 106533, 944 base pairs (bp) of the 3' UTR were used. For the DSL contig 914, a 494 bp long internal fragment comprising a partial DSL domain and three EGF-repeats was used. For the DSL contig 258, a 2108 bp long 3' fragment covering a partial DSL and three EGF domains was used. For the DSL contig 27364, an 866 bp long 3' fragment including a partial DSL domain and five EGF-repeats was used. For the DSL contig 85017, a 980 bp long internal fragment comprising one DSL and two EGF domains was used. For the DSL contig 8347, a 1007 bp long internal fragment encoding for one DSL domain and four EGF repeats was used. For the DSL contig 198457, a 2903 bp long 3' fragment covering the DSL domain, nine EGF repeats and the transmembrane domain was used. For the DSL contig 221422, a 1481 bp long 3' fragment spanning a partial DSL, three EGF and the transmembrane domain was used. For the DSL contig 111341, an internal fragment of $2521 \mathrm{bp}$ length encoding 19 EGF repeats was used. For the DSL contig 64109, 1504 bp of the 3' UTR were used. Despite the lack of an open reading frame, $702 \mathrm{bp}$ were used to generate a riboprobe against the transcribed DSL contig 10061. For the LNG contig 51336, internal 903 bp covering the DUF domain were used for riboprobe synthesis. For the LNG contig 32233, 950 bp of the 3' UTR were used. For the hes contig 1791, a 1725 bp long 3' fragment comprising the Orange domain was used. For the hes contig 366559, a 2066 bp long 3' fragment that included the bHLH and Orange 
domains was used. For the fringe-like contig 15835, a $912 \mathrm{bp}$ fragment covering a part of the fringe domain was used. For the CSL contig 165301, a 970 bp long 3 ' fragment starting in the Lag1 domain was used.

For all genes except fringe, whole mount in situ hybridisation was performed as described in chapter 3 on at least three developmental stages, namely $29 \mathrm{hpfc}$, $42 \mathrm{hpfc}$ and $57 \mathrm{hpfc}$. In all experiments, detection of $\beta$-tubulin expression was performed as positive control. For all genes, parallel reactions with an optional NAC treatment were additionally included. Samples were embedded in $60 \%$ Glycerol or in a 1:2 mixture of benzyl benzoate and benzyl alcohol (BB:BA) and photodocumented on a Zeiss microscope Axio Imager Z1. For each sample, several images were captured at different focal planes and for some embryos projected using Macnification version 2.0.1. All images were edited in Adobe Photoshop CS3 version 10.0.1 by adjusting the entire image for brightness and contrast.

\section{Pharmacological inhibition of Notch signalling}

Freshly deposited egg masses were collected and individual egg capsules were retrieved as described in chapter 2 . Their development was monitored to determine the time point of the first cleavage. DAPT (Sigma, \#D5942) was dissolved in dimethyl sulfoxide (DMSO) to prepare a $23 \mathrm{mM}$ DAPT stock solution. The egg capsules of an individual egg mass were divided into two or three groups. Maximum 25 egg capsules per group were transferred in 12-well tissue culture plates and incubated in $1.5 \mathrm{~mL}$ DAPT solution or snail water [16] at $25^{\circ} \mathrm{C}$. In most experiments, an additional control (DMSO diluted to the corresponding concentration in the DAPT treatment) was included. The embryos were treated during the first or second day of development as summarised in tables 2 and 3. The first day of development corresponds to the cleavage phase and the onset of gastrulation [17]. The embryos were treated with $10 \mu \mathrm{M}, 50 \mu \mathrm{M}$ or $100 \mu \mathrm{M}$ DAPT for several durations. During the second day of development, embryos of L. stagnalis complete gastrulation and develop into trochophore larvae with a differentiated shell field (see chapter 2). The embryos were treated with $50 \mu \mathrm{M}, 100 \mu \mathrm{M}, 150 \mu \mathrm{M}$ and $200 \mu \mathrm{M}$ filtered or unfiltered DAPT for different time windows around the presumably shell field specifying contact event at $29 \mathrm{hpfc}$. The onset and durations of individual treatments are provided in table 3 . All embryos were allowed to develop into veliger larvae and were fixed at around $75 \mathrm{hpfc}$, at $100 \mathrm{hpfc}$ or at nine days post first cleavage (dpfc). All media were at least once completely exchanged. Fixed larvae 
were assayed for endogenous alkaline phosphatase (AP) activity as described in chapter 2. Samples were mounted in 60\% Glycerol or BB:BA and viewed and photographed using a Zeiss microscope Axio Imager Z1. All images were edited in Adobe Photoshop CS3 version 10.0.1 by adjusting the entire image for brightness and contrast.

\section{Results}

Identified Notch signalling pathway components in L. stagnalis

In total, 17 Notch pathway members that are encoded by distinct genomic loci were recovered (Fig. 2). Among those, 11 unique sequences represent transcripts with conserved domains considered to be diagnostic for DSL ligands [7]. Precisely, internal coding sequences of $494 \mathrm{bp}$ length (contig 914), $1007 \mathrm{bp}$ length (contig 8347), 3156 bp length (contig 111341) and 2227 bp length (contig 85017) were isolated. The latter reveals an unusual domain arrangement with three DSL domains each followed by two or three EGF repeats (Fig. 2). Another 702 bp long DSL transcript (contig 10061) does not comprise an open reading frame. For three other DSL transcripts, 3 ' fragments of 4378 bp length with 2641 bp UTR (contig 106533), 866 bp length comprising 126 bp UTR (contig 27364) and 1481 bp length with a 479 bp long UTR (contig 221422) were isolated. Finally, the full transcripts of three additional DSL ligands were cloned. Two transcripts resemble Delta proteins. The first transcript is $3127 \mathrm{bp}$ long and consists of an open reading frame of $1965 \mathrm{bp}$ length that lacks the MNNL domain (contig 258). The second delta transcript (contig 198457 ) is $3673 \mathrm{bp}$ long with a $2658 \mathrm{bp}$ long open reading frame. The third fully isolated gene (contig 64109) represents a jagged orthologue as revealed by the presence of a VWV domain. The total length is $7608 \mathrm{bp}$ with an open reading frame of 3669 bp length.

Two of the 17 recovered transcripts encode Notch receptors. The 3' fragments of these transcripts are $3271 \mathrm{bp}$ long, $126 \mathrm{bp}$ of which untranslated (contig 51336), and 6691 bp long with a 2516 bp long 3' UTR (contig 32233). An orthologue of the CSL DNA-binding protein was retrieved as well, which comprised a 2172 bp long open reading frame within a $2270 \mathrm{bp}$ long transcript. Also, an internal transcript of $987 \mathrm{bp}$ length reveals similarity to the Notch modulator Fringe. Finally, the 3 ' regions of two transcripts that encode for Notch target genes of the hes complex 


\section{DSL proteins}

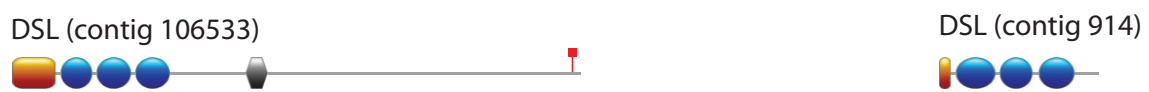

DSL (contig 258)

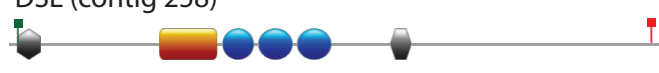

DSL (contig 85107)

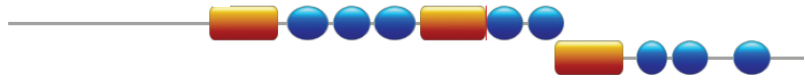

DSL (contig 198457)

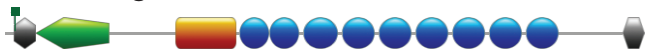

1

DSL (contig 27364)

00000 II

DSL (contig 8347)

$\mathrm{OOO}$

DSL (contig 221422)

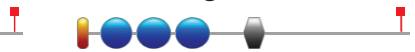

DSL (contig 111341)

$0000000000000 \quad 00000$

DSL (64109)

0000000000000000

\section{LNG proteins}

LNG (contig 51336)

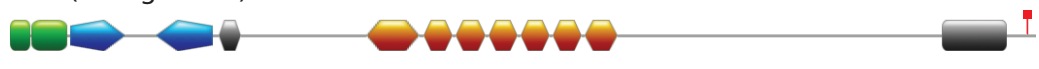

LNG (contig 32233)

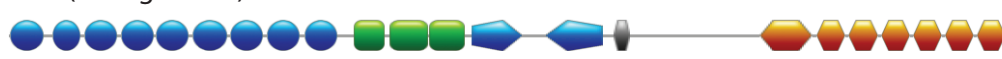

further Notch pathway components

Hey (contig 1791)

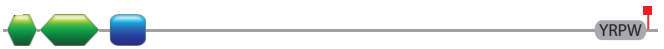

Hes (contig 366599)

$\longrightarrow$ WRPW' fringe (contig 15835)

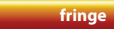

\begin{tabular}{llll}
\hline DSL domain & LNR repeat & Orange domain \\
Epidermal growth factor-like repeat & Notch protein domain A (NOD) \\
transmembrane helix & Notch protein domain B (NODP) \\
Signal peptide & Ankyrin repeat & Lag1 & Lag1 DNA binding domain \\
MNNL domain & DUF3454, Notch & Beta-trefoil DNA binding domain \\
van Willebrand factor C & Helix-loop-Helix domain & Immunglobulin E set \\
\hline
\end{tabular}

Fig. 2. Domain arrangement of Notch pathway orthologues in L. stagnalis. The coding parts of isolated transcripts are depicted. Schematics are to scale. 
were isolated. One transcript was 2388 bp long with an 880 bp long 3' UTR (contig 366599) and included the C-terminal WRPW motif of Hes proteins. The second transcript was 3639 bp long, $67 \mathrm{bp}$ of which correspond to the 3' UTR (contig 1791), and comprised an YRPW motif characteristic for hes-related hey genes.

The spatial expression of Notch pathway components during the early shell field development

The expression of the identified orthologues of the Notch receptors, ligands and the effector during the early shell field development was examined at three key stages. The first time point at $29 \mathrm{hpfc}$ reflects the contact establishment between the future shell forming dorsal ectoderm and the underlying archenteron. The second time point corresponds to the beginning invagination of the specified shell field and the formation of the shell gland at $42 \mathrm{hpfc}$. Finally, at $52 \mathrm{hpfc}$ the first secretion of the insoluble shell material can be observed (see chapter 2). None of the studied Notch ligands was expressed in the dorsal ectoderm or the contacting endoderm at $29 \mathrm{hpfc}$ (Fig. 3). Similarly, no expression of the Notch receptors and the CSL effector was observed at the contact zone (Fig. 4A and G). Also, none of the studied genes was expressed in the vicinity of the forming shell gland at $42 \mathrm{hpfc}$ and in or near the invaginated shell gland at $52 \mathrm{hpfc}$ (data not shown; see also Fig. 4).

Notch pathway orthologues are expressed in the anterior ectoderm

Strong expression of one Notch receptor (contig 51366) was found in cells of the anterior ectoderm and the most anterior dorsal ectoderm at $29 \mathrm{hpfc}$ (Fig. 4A and B). At the same stage, weak expression of the CSL DNA-binding protein (contig 165301) was localised in the same area (Fig. 4G and H). At $42 \mathrm{hpfc}$, the expression of these two genes was confined to the anterior head vesicle cells and the prototroch (Fig. 4C, D, I and J). At $52 \mathrm{hpfc}$, both genes were still expressed in these tissues (Fig. 4E, F, K and L).

\section{Hes/hey gene expression in the early veliger larva}

The expression of the delta orthologue contig 198457 between $55 \mathrm{hpfc}$ and $62 \mathrm{hpfc}$ as well as the expression of both the isolated hes and hey genes between $57 \mathrm{hpfc}$ and $76 \mathrm{hpfc}$ were examined. No expression of the delta gene was observed whereas the hes and hey genes were both continuously expressed in the same tissues (Fig. 5). Three distinct expression domains could be distinguished. Firstly, both genes were 
expressed in two rows of cells at the shell field periphery (Fig. 5, pink arrowheads). Secondly, hes/hey expression was also found in cells surrounding the opening of the stomodeum and in three individual cells left, right and anterior from the stomodeal opening (Fig. 5, yellow arrowheads). Finally, the anterior head vesicle cells were found to express both the hes and the hey orthologue (Fig. 5, blue arrowheads).
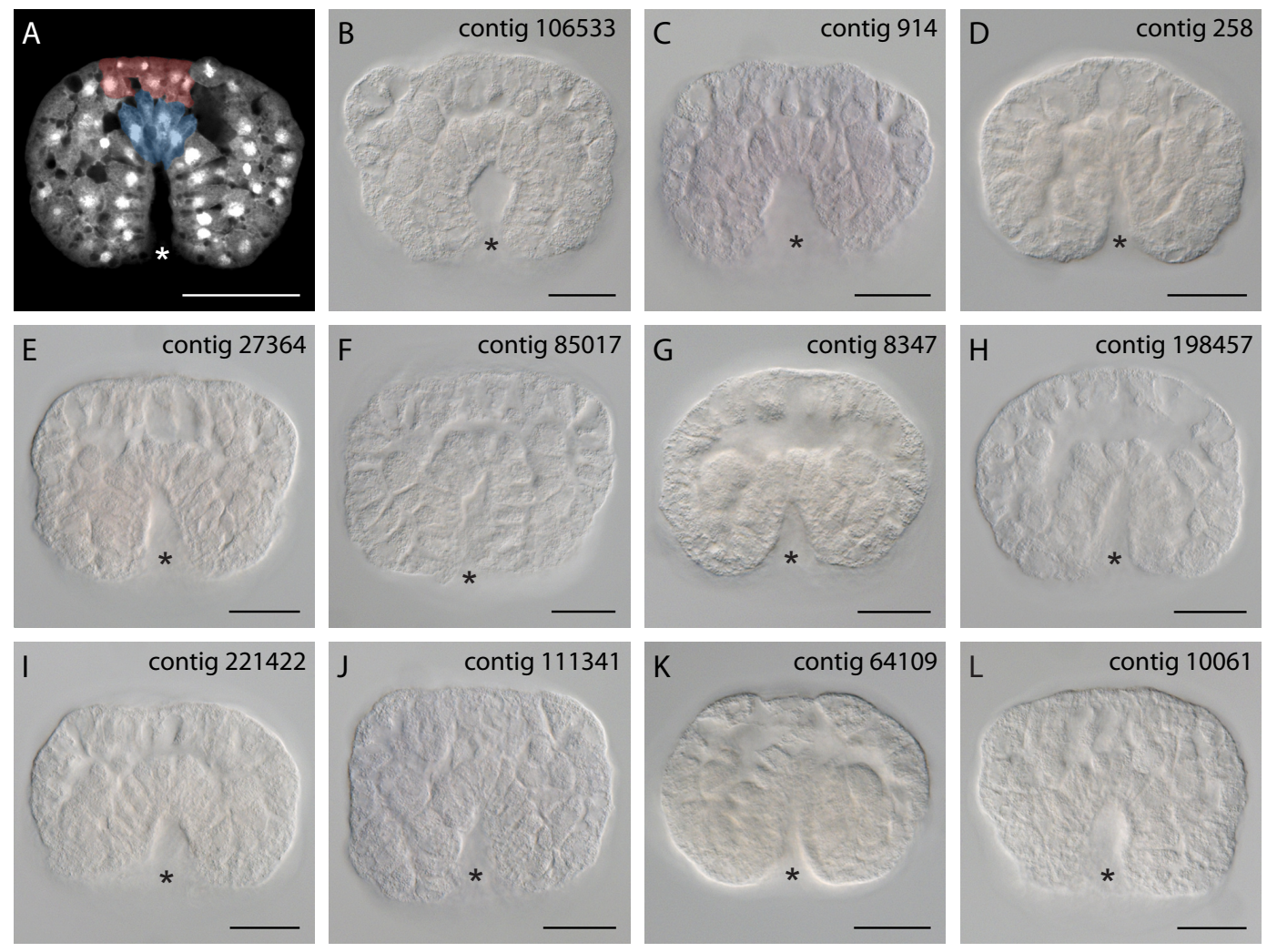

Fig. 3. DSL ligands are not expressed in the contact zone between future shell field cells and the underlying endoderm. A At $29 \mathrm{hpfc}$, cells at the tip of the invaginating archenteron (blue) contact dorsal ectodermal cells (red) that give rise to the shell field. B-L None of the identified transcripts encoding Notch ligands is expressed in $29 \mathrm{hpfc}$ old embryos. All embryos are oriented with the prospective shell field to the top. An asterisk marks the position of the blastopore. All scale bars are $50 \mu \mathrm{m}$. 
Chapter 4
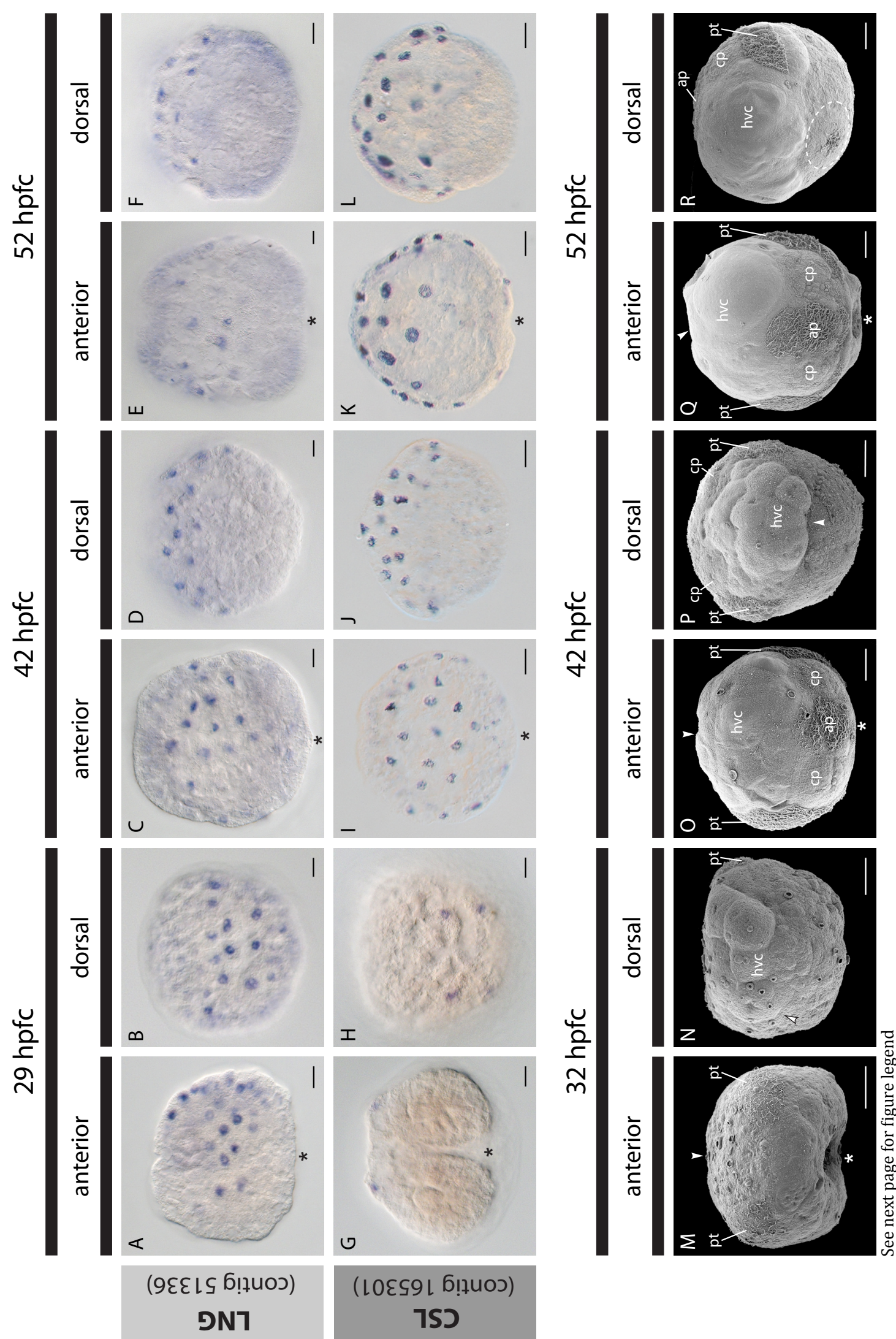
Fig. 4. Corresponding notch and CSL expression patterns in the trochophore suggest an employment of Notch signalling during early neurogenesis in L. stagnalis. The expression of the Notch receptor encoded by contig 51336 (A-F) and the CSL DNA-binding protein encoded by contig 165301 (G-L) is situated in the anterior-dorsal ectoderm at $29 \mathrm{hpfc}(\mathrm{A}, \mathrm{B}, \mathrm{G}$ and $\mathrm{H})$ and is confined to the anterior head vesicle cells at $42 \mathrm{hpfc}(\mathrm{C}, \mathrm{D}$; I and J) and $52 \mathrm{hpfc}$ (E, F, K and L). M-R are SEM micrographs of corresponding developmental stages illustrating the main larval structures. All embryos viewed from an anterior perspective are oriented with the shell field to the top. Dorsal views are oriented with anterior to the top. An asterisk marks the position of the blastopore. The position of the shell field is marked by arrowheads in $\mathrm{M}-\mathrm{Q}$ and the dashed oval in R. Abbreviations: $\mathrm{ap}=$ apical plate, $\mathrm{cp}=$ cephalic plate, $\mathrm{hvc}=$ head vesicle cells, $\mathrm{pt}=$ prototroch. Panel $\mathrm{R}$ is reflected about the vertical axis for consistency of presentation. All scale bars are $50 \mu \mathrm{m}$.

Pharmacological inhibition of Notch signalling during the early development of L. stagnalis

Since it is possible that not all notch and $d s l$ paralogues in L. stagnalis were isolated, Notch signalling was also pharmacologically inhibited. The larvae were treated with the Notch-specific inhibitor DAPT before, during and after the initial establishment of the presumably specifying contact to further assess the putative involvement of Notch signalling in shell field specification. Additionally, embryos were treated before the formation of the contact during cleavage and early gastrulation to prove whether potential shell field-phenotypes can be correlated with the contact event. Most embryos were assayed for endogenous AP activity at around $75 \mathrm{hpfc}$.

Several abnormalities were observed in embryos, which were treated during the first day of development (table 1, Figs. 6 and 7). Most obviously, abnormally small embryos were frequently observed in the treatment group but rarely in the corresponding controls. In these embryos (which were treated before shell field specification takes place), alterations in the shell gland appearance were also commonly observed. The shell gland of several larvae was less far developed than in control embryos. The outer AP-positive ring present in control embryos was absent in these immature shell glands and their shape was rather oval (shorter along the anteriorposterior axis) as typical for earlier shell gland stages (see chapter 2, Fig. 4). In one embryo, which developed until $100 \mathrm{hpfc}$, an ovally shaped shell gland failed to evaginate (Fig. 6C). In another otherwise normal embryo, the invaginated part of the shell gland was not connected to the outer part and instead was situated laterally under the left protonephridium near the prototroch (Fig. 6A and B).

Frequently observed severe abnormalities were large, apparently cell-free spaces (Fig. 7, black x's). These blister-like malformations seemed to be located in the anterior half of the embryo (Fig. 7B), particularly below the head vesicle cells 
(Fig. 7A and B) or under the ciliated prototrochal cells (Fig. 7F) and were associated with a relatively small posttrochal region (the area that comprises the shell field and the surrounding ectoderm; Fig. 7B and F). The larval liver cells that occupy most of the internal space of the embryo (Fig. 8) [17] were not differentiated in these embryos (Fig. 7C cf. G). Rarely, unidentifiable ectodermal outgrowths occurred (Fig. 7D, black arrowhead). None of these malformations were observed in control embryos that were reared in DMSO or water.

In contrast, DAPT-treatment during the second day of development when the shell field is presumably specified, did not lead to abnormal development in more than 200 larvae from 24 egg masses (table 2). No difference was observed between the treatment and control groups. Both the general development and the shell field of DAPT-treated embryos appeared unaltered as revealed by endogenous AP activity.

Table 1. Summary of DAPT treatments during the first day of development in L. stagnalis.

\begin{tabular}{|c|c|c|c|c|c|c|}
\hline $\begin{array}{l}\text { treatment } \\
\text { start (hpfc) }\end{array}$ & $\begin{array}{l}\text { treatment } \\
\text { stop (hpfc) }\end{array}$ & $\begin{array}{c}\text { concentration } \\
(\mu \mathrm{M})\end{array}$ & $\begin{array}{l}\text { No. of } \\
\text { exps. }\end{array}$ & $\begin{array}{l}\text { total no. } \\
\text { of treated } \\
\text { embryos }\end{array}$ & $\begin{array}{c}\text { altered } \\
\text { phenotypes } \\
\text { \% (no.) }\end{array}$ & $\begin{array}{c}\text { severe } \\
\text { phenotypes }^{\dagger} \\
\text { \% (no.) }\end{array}$ \\
\hline 0 & 23 & 100 & 1 & 11 & $0(0)$ & $0(0)$ \\
\hline \multirow[t]{3}{*}{2} & 6 & 100 & 1 & 11 & $0(0)$ & $0(0)$ \\
\hline & 9 & 100 & $1^{*}$ & 15 & $6.7(1)$ & $0(0)$ \\
\hline & 18 & 100 & $1^{*}$ & 14 & $0(0)$ & $0(0)$ \\
\hline \multirow[t]{8}{*}{3} & 6 & 100 & 2 & 26 & $3.8(1)$ & $0(0)$ \\
\hline & 9 & 100 & $1^{*}$ & 8 & $37.5(3)$ & $25(2)$ \\
\hline & 12 & 100 & $1^{*}$ & 10 & $0(0)$ & $0(0)$ \\
\hline & 16 & 100 & $3^{*}$ & 40 & $\sim 40(\sim 16)$ & $20(8)$ \\
\hline & 18 & 100 & 1 & 15 & $13.3(2)$ & $13.3(2)$ \\
\hline & 20 & 100 & 1 & 25 & $24(6)$ & $12(3)$ \\
\hline & 22 & 50 & 1 & 6 & $83.3(5)$ & $33.3(2)$ \\
\hline & 22 & 10 & 1 & 6 & $0(0)$ & $0(0)$ \\
\hline 4 & 27 & 100 & $1^{*}$ & 21 & $19(4)$ & $9.5(2)$ \\
\hline 5 & 9 & 100 & $2 *$ & 10 & $0(0)$ & $0(0)$ \\
\hline \multirow[t]{2}{*}{6} & 19 & 100 & 1 & 10 & $20(2)$ & $20(2)$ \\
\hline & 22 & 100 & 1 & 18 & $16.7(3)$ & $0(0)$ \\
\hline \multirow[t]{2}{*}{7} & 22 & 100 & $1^{*}$ & - & (2) & $(1)$ \\
\hline & 29 & 100 & $1^{*}$ & 10 & $10(1)$ & $0(0)$ \\
\hline 9 & 20 & 100 & $2^{*}$ & $5(-)$ & $(1)$ & $0(0)$ \\
\hline 15 & 28 & 100 & 1 & 12 & $0(0)$ & $0(0)$ \\
\hline
\end{tabular}

* each one experiment included water as single control. †severe phenotypes refer to embryos with blister-like malformations or ectodermal outgrowths. 

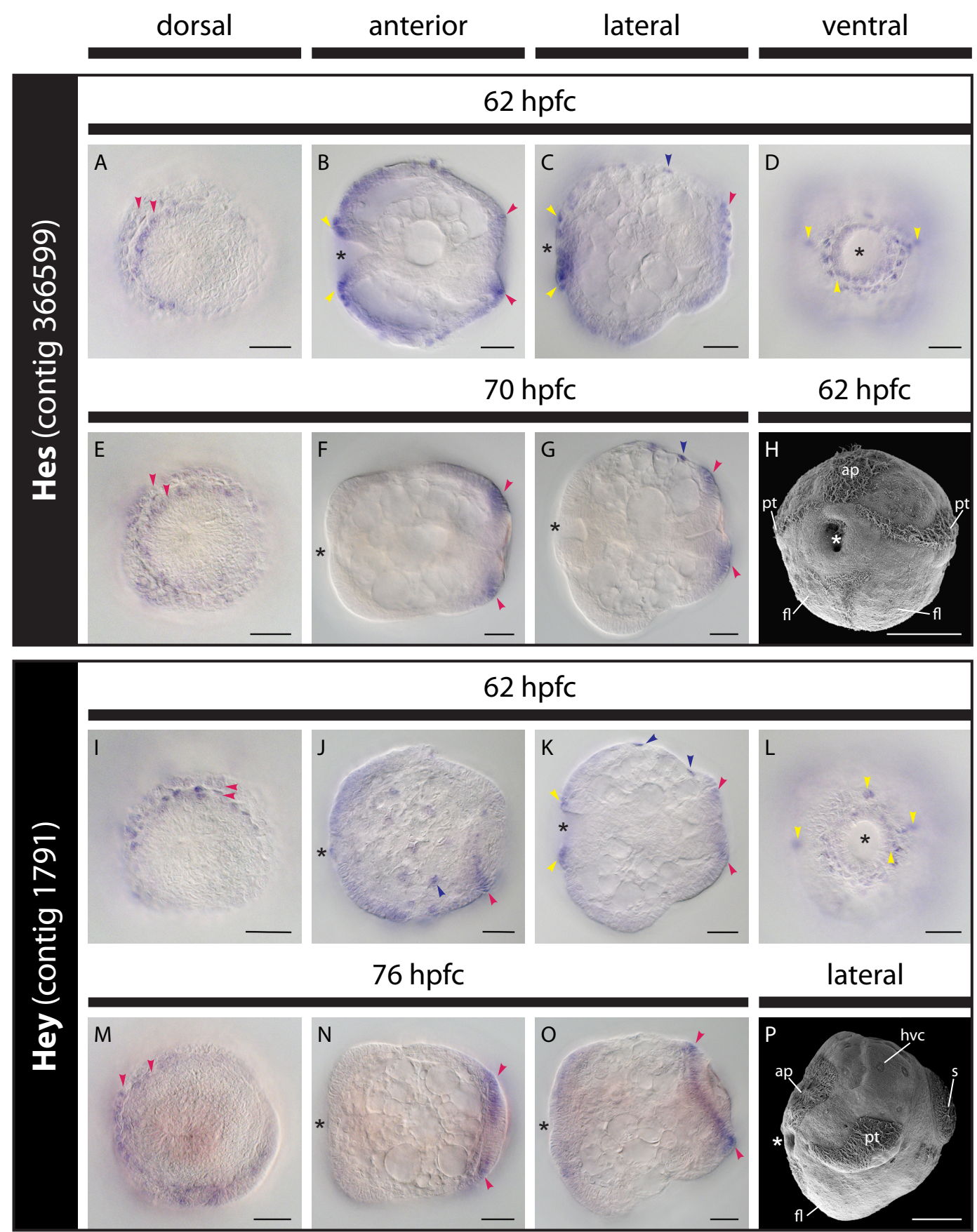

Fig. 5. Notch target genes reveal overlapping expression domains in the early veliger larva.

Transcripts encoding the Hes DNA-binding protein contig 366599 (A-G) and the Hey protein contig 1791 (I-0) are continuously expressed in the shell field periphery (pink arrowheads), around the stomodeal opening (yellow arrowheads) and the anterior head vesicle cells (blue arrowheads). The SEM in $\mathbf{H}$ depicts a ventral view of a $62 \mathrm{hpfc}$ old larva with the main larval structures indicated. $\mathbf{P}$ is a SEM from a lateral perspective of a $\sim 70 \mathrm{hpfc}$ old veliger larva. Larvae seen from an anterior or lateral perspective are oriented with the shell field to the right. Dorsal or ventral views are oriented with anterior to the top. An asterisk marks the position of the blastopore. Abbreviations: ap= apical plate, $\mathrm{fl}=$ foot lobe, $\mathrm{hvc}=$ head vesicle cells, $\mathrm{pt}=$ prototroch, $\mathrm{s}=$ shell. All scale bars are $50 \mu \mathrm{m}$. 


\section{Discussion}

Notch pathway composition in L. stagnalis

The present study focuses on the main molecules that function in Notch-mediated signal transduction, the DSL ligands and the Notch receptors whose interaction evokes active signalling. The products of additionally isolated genes act on various levels of the Notch signalling cascade; they comprise the DNA-binding CSL protein that functions as Notch effector, downstream target genes of the hairy/enhancer of split complex and the glycosyltransferase Fringe which is involved in the posttranslational activation of the Notch receptor. All transcripts were identified based on previously defined diagnostic domain compositions [7]. For instance, these were the combined presence of DSL domain and EGF repeats for DSL ligands whereas LNG domains co-occurring with ankyrin repeats were considered diagnostic for Notch receptors.

Among the seventeen isolated Notch pathway elements, eleven are orthologues of DSL ligands. Both ligand families are represented although the four internal DSL fragments cannot be designated with certainty to either the Delta or Jagged/Serrate type ligands. These two ligand types can be distinguished by different numbers of EGF repeats and the presence of a VWC domain in Jagged proteins [7]. The transcript revealing a VWC domain (contig 64109) clearly represents a jagged orthologue, and the nineteen EGF repeats comprised by the partial contig 111341 suggest the presence of a second jagged in L. stagnalis (Fig. 2). This is in congruence with the numbers of jagged genes found in other lophotrochozoan taxa (two jagged copies in the gastropod Lottia gigantea and one copy in the annelid Helobdella robusta [7]). The other two fully isolated transcripts can be classified as delta orthologues. One reveals the classical structure of metazoan Delta proteins (contig 198457) while the other one contains only three EGF repeats and lacks the MNNL domain (contig 258), both features that have been reported to be common in lophotrochozoan delta proteins [7]. Also, the lack of VWC domains in the three 3 ' fragments indicates that they also encode Delta proteins (contigs 106533, 27364 and 221422). Among the remaining four DSL transcripts, one lacks an open reading frame and might represent a recent pseudogene (contig 10061) whereas another transcript (contig 85107) reveals three DSL domains each followed by two or three EGF repeats (Fig. 2). Such a repetitive organisation has not been reported in DSL proteins. In the genome of Nematostella vectensis, genes composed of repeated DSL 
domains were identified [7], but these genes do not include intermediate EGF repeats. Contig 85107 in L. stagnalis might have arisen by the same genetic mechanisms that are proposed to have generated the varying numbers of EGF repeats in DSL proteins (exon shuffling, tandem duplication or DNA slippage [7]). It is not clear whether this transcript represents a precursor mRNA which is subject to alternative splicing or a functional Notch ligand.

In general, the number of DSL paralogues in L. stagnalis is remarkably high compared to most of the few other studied protostomes such as H. robusta, Aedes aegypti and Caenorhabditis elegans [7]. Even under exclusion of the putative pseudogene and the repetitively structured transcript, nine distinct genomic loci remain. This corresponds to the number of DSL genes found in the early branching gastropod L. gigantea [7]. The same holds true for the Notch receptors in these species compared to L. stagnalis (each one or two copies). While the many isolated $d s l$ paralogues may suggest that most of the ligands were recovered, none of them is coexpressed with the notch and csl orthologues in the trochophore (Fig. 3 cf. Fig. 4A and G). Thus, it is possible that further unknown $d s l$ paralogues exist in L. stagnalis.

Notch signalling does not specify the shell field in L. stagnalis

The isolated Notch pathway orthologues in L. stagnalis encode representatives of the main components of canonical Notch signalling. In particular, the Notch receptors and DSL ligands are the core proteins whose interaction leads to the functional switch of the DNA-binding CSL protein from a transcriptional repressor to an activator (Fig. 1). Therefore, these transcripts might be considered to reflect the main phases of active canonical Notch signalling. Their expression at critical transitions during the early shell field development was investigated, precisely during the establishment of the presumably specifying contact, the initial shell gland invagination and the beginning shell secretion. None of these genes was found to be expressed in or near cells or tissues that are involved in these crucial events. Furthermore, the pharmacological inhibition of Notch signalling during the period of contact formation had no observable effect (table 2). In all treated embryos an apparently normal shell gland developed (data not shown). However, disruption of Notch signal transduction earlier in development (well before the shell field is presumptively specified) did affect the normal embryogenesis (Figs. 6 and 7). This indicates the successful delivery of the DAPT inhibitor. Therefore, Notch signalling 
does not seem to be involved in the specification of the shell field in L. stagnalis, but might exert indirect effects on the subsequent differentiation.

This is in contrast to previous observations made in the early branching gastropod $H$. asinina. Here, an orthologue of the DSL ligand delta is continuously expressed in the dorsal ectoderm before and after the first shell field differentiation. Although these two gastropod species are distantly related [18], the shell field-associated expression of well-studied genes like engrailed is conserved even among molluscan classes (see general introduction, pp. 11). Deployment of the Notch pathway in H. asinina but not in L stagnalis highlights modifications in the genetic regulation of the deeply conserved process of molluscan shell field formation. These might translate into variations of the morphogenetic events associated with early shell formation in these two species. Since the shell field morphogenesis in H. asinina has not yet been studied in detail, it is not possible to compare the morphological conditions under which shell field development is initially realised in these two species.

The present study cannot completely rule out an involvement of Notch signalling in the early shell field formation of L. stagnalis. In the absence of an annotated genome, it cannot be excluded that so far unidentified genes encoding further Notch receptors, ligands and effectors exist.

Table 2. Summary of DAPT treatments during the initial shell field formation in L. stagnalis

\begin{tabular}{|c|c|c|c|c|c|c|}
\hline $\begin{array}{l}\text { treatment } \\
\text { start (hpfc) }\end{array}$ & $\begin{array}{l}\text { treatment } \\
\text { stop (hpfc) }\end{array}$ & $\begin{array}{c}\text { concentration } \\
(\mu \mathrm{M})\end{array}$ & $\begin{array}{l}\text { No. of } \\
\text { exps. }\end{array}$ & $\begin{array}{c}\text { no. of egg } \\
\text { masses }\end{array}$ & $\begin{array}{l}\sim \text { no. of } \\
\text { embryos }\end{array}$ & $\begin{array}{c}\text { altered } \\
\text { phenotypes } \\
\% \text { (no.) }\end{array}$ \\
\hline 15 & 38 & 100 & 1 & 3 & 21 & $0(0)$ \\
\hline \multirow{4}{*}{17} & \multirow{4}{*}{45} & 50 & \multirow{4}{*}{2} & \multirow{4}{*}{11} & 14 & $0(0)$ \\
\hline & & 100 & & & 28 & $0(0)$ \\
\hline & & 150 & & & 22 & $0(0)$ \\
\hline & & 200 & & & 31 & $0(0)$ \\
\hline 23 & 44 & 100 & 3 & 5 & 45 & $0(0)$ \\
\hline \multirow{5}{*}{27} & \multirow{5}{*}{34} & 0.1 & \multirow{5}{*}{1} & \multirow{5}{*}{3} & 6 & $0(0)$ \\
\hline & & 1 & & & 6 & $0(0)$ \\
\hline & & 10 & & & 6 & $0(0)$ \\
\hline & & 50 & & & 12 & $0(0)$ \\
\hline & & 100 & & & 12 & $0(0)$ \\
\hline \multirow{4}{*}{30} & \multirow{4}{*}{39} & 0.1 & \multirow{4}{*}{1} & \multirow{4}{*}{2} & 6 & $0(0)$ \\
\hline & & 1 & & & 6 & $0(0)$ \\
\hline & & 10 & & & 6 & $0(0)$ \\
\hline & & 100 & & & 12 & $0(0)$ \\
\hline
\end{tabular}




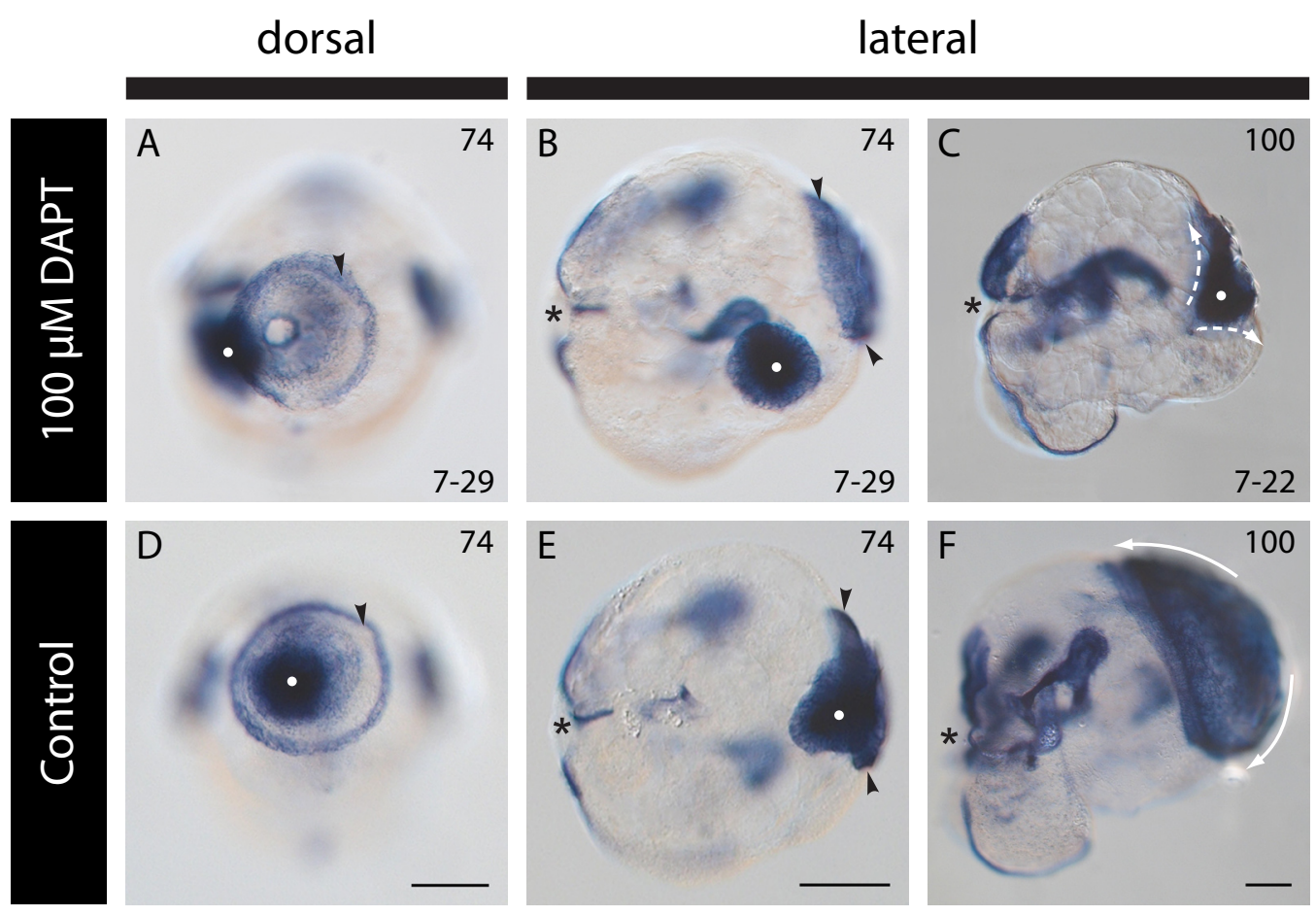

Fig. 6. Rare shell field abnormalities displayed by DAPT-treated larvae. In otherwise normally developed and intact embryo, the invaginated part of the shell gland is dislocated to the right side (white dots in A and B). The evaginated part of the shell field (black arrowheads in A and B) remains dorsal and is not altered compared to control embryos (black arrows in $\mathbf{D}$ and $\mathbf{E}$ ). The embryo in $\mathbf{C}$ displays a malformed shell gland, which failed to evaginate (dashed arrows) and to expand as can be observed in control embryos (white arrows in F). All larvae are oriented with anterior to the top. A black asterisk marks the position of the blastopore. A white dot marks the position of the invaginated shell gland lumen. Numbers in the lower left corner of each panel indicate the treatment period in hours post first cleavage (hpfc). Numbers in the upper right corner of each panel denote the age in hpfc. Panels $\mathrm{C}$ and $\mathrm{F}$ are reflected about the vertical axis for consistency of presentation. Control and treatment groups are to scale. All scale bars are $50 \mu \mathrm{m}$.

\section{Shell field-unrelated Notch signalling during the early larval development}

Although none of the studied Notch pathway components was expressed in early shell field stages, some of these genes were expressed in other tissues or the evaginating shell gland (Figs. 4 and 5).

For instance, cells of the anterior ectoderm revealed persistent expression of one Notch receptor (Fig. 4A-F) and the CSL effector (Fig. 4G-L). At 29 hpfc, the Notch receptor is expressed in cells of the anterior (animal) and dorsal ectoderm (Fig. 4A and $\mathrm{B}$ ). The initially strong notch expression is at $52 \mathrm{hpfc}$ restricted to fewer cells that reveal weaker expression (Fig. 4A-D cf. E and F). The Notch pathway effector $C S L$ is persistently co-expressed in the same tissue. In contrast to notch, the number of cells and the strength of the csl expression increase until 52 hpfc (Fig. 4G and H). No corresponding spatial expression of one of the isolated DSL ligands was detected 


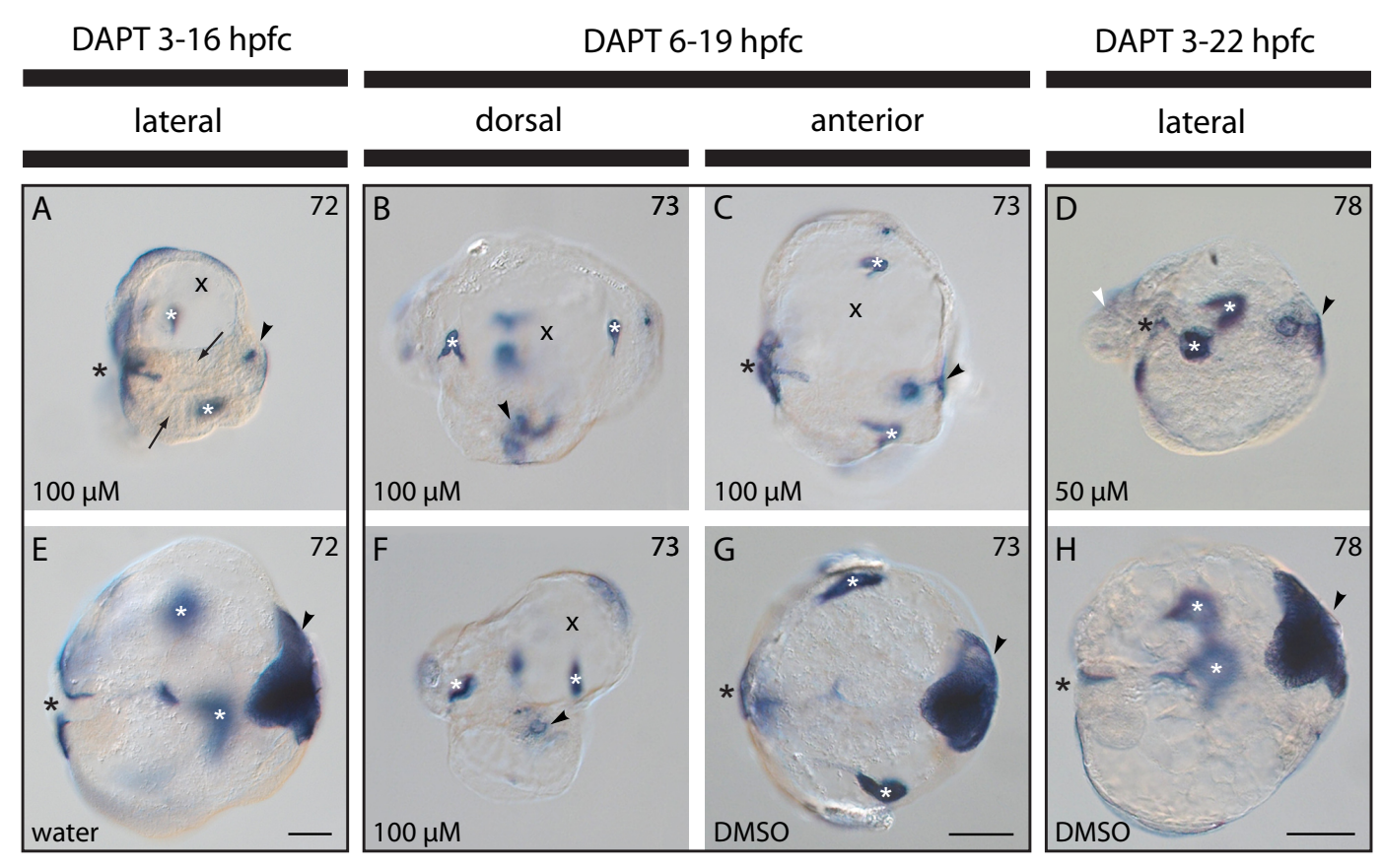

Fig. 7. Observed phenotypes after inhibition of Notch signalling during the first day of development. Large, apparently cell-free spaces (indicated by " $\mathrm{x}$ " in A, B, C and F) are commonly present in DAPT-treated larvae while the large-vacuolar endodermal cells (larval liver cells) that occupy most of the internal space in control embryos ( $\mathbf{E}$ and $\mathbf{H}$ ) were not fully differentiated (A, black arrows). Excessive outgrowths (white arrowhead in D) were rarely observed. An invaginated shell field is always present (black arrowheads). Panels B and C are different perspectives on the same embryo. The larvae in A, B, D, E, F and $\mathbf{H}$ are oriented with anterior to the top. The larvae in $\mathrm{C}$ and $\mathrm{G}$ are oriented with right to the top. A black asterisk marks the position of the blastopore. White asterisks mark the positions of the protonephridia. The applied DAPT concentration is indicated in the lower left corner of panels A-D and F. Numbers in the upper right corner of each panel denote the age in hours post first cleavage (hpfc). Panels $\mathrm{A}$ and $\mathrm{H}$ are reflected about the vertical axis for consistency of presentation. Control and treatment groups are to scale. All scale bars are $50 \mu \mathrm{m}$.

at the trochophore stage. This could be explained by the presence of further $d s l$ paralogues in L. stagnalis that were not recovered. Alternatively, one of the identified DSL ligands is involved in this process but is expressed in a temporally strictly regulated way, which was not revealed at the investigated time points. In fact, spatiotemporal regulation of the active signalling process is more often achieved by strict regulation of the ligand's expression and by receptor protein modification than by localised receptor expression [19]. Therefore, the expression of a notch orthologue in a given tissue is not equivalent to simultaneous active signalling. The same holds true for the CSL effector, which serves a dual function of repressing and activating the transcription of target genes. The cells that express notch and csl are the progeny of the first quartet of micromeres, precisely $1 \mathrm{a}, 1 \mathrm{c}$ and $1 \mathrm{~d}$ (reviewed in [17]). During the second day of development, these anterior ectodermal cells first differentiate into the head vesicle cells of the trochophore larva (Fig. 4N-R) and 
subsequently into the cephalic vesicle of the 2+dpfc veliger larva [17]. In the gastropod Crepidula furnicata, the cell lineages of the first quartet of micromeres contribute to larval neural structures such as the apical organ [20]. Therefore, the expression of notch and csl in ectodermal head vesicle cells of L. stagnalis might reflect the beginning neurogenesis and possibly represents another example of the well-known function of Notch signalling in regulating the number of cells that acquire neural potential via lateral inhibition (e.g. [10, 11, 21]).

A neurogenic function for the Notch pathway during the larval development of L. stagnalis is further corroborated by the expression of known Notch targets of the hairy/enhancer of split gene family. In several vertebrates and arthropods, Hes transcription factors function in the inhibition of neurogenesis by repressing the transcriptional activation of proneural genes in neural precursor cells (reviewed in [22]). During the third day of development, both isolated hes and hey genes are coexpressed in the anterior head vesicle cells (Fig. 5C, G and K, blue arrowheads). Although Hes proteins function both Notch-dependent and Notch-independent [22], it seems likely that the hes and hey orthologues are expressed in the anterior head vesicle cells in response to earlier Notch signalling.

Both genes are also expressed in three rows of cells surrounding the stomodeal opening and three individual cells dorsolateral and anterior from the mouth opening around 62 hpfc (Fig. 4B-D, K and L, yellow arrowheads). The identity and further development of the hes and hey expressing cells in L. stagnalis is not clear. Early neurons are formed near the stomodeal opening at this stage [23, 24]. However, a neurogenic function of hes and hey in the cells around the stomodeal opening remains speculative.

Finally, between $62 \mathrm{hpfc}$ and $76 \mathrm{hpfc}$, both hes and hey orthologues are persistently expressed at the shell field periphery (Fig. 5, red arrowheads), precisely in at least two adjacent cell rows. These include the highly elongated cells that secrete the first insoluble shell material (see chapter 2). Hes proteins are involved in boundary formation during vertebrate neurogenesis (reviewed in [22]) while Notch signalling is commonly deployed in the establishment of compartment boundaries $[9,10]$. The cellular arrangements at the shell field periphery where the hes and hey orthologues are expressed represent a compartment boundary [25].

Hes and Hey transcription factors are known to form heterodimers [22]. The constant co-expression of contig 366599 and contig 1791 suggests that the isolated hes and hey orthologues in L. stagnalis also act in form of a heterodimer. 


\section{Inhibition of Notch signalling during cleavage and early gastrulation}

The pharmacological inhibition of Notch signalling between three and $22 \mathrm{hpfc}$ resulted in several developmental abnormalities. While some of these represent rare or unique misdevelopments (the "misplaced" invaginated part of the shell gland and the ectodermal outgrowths; Figs. 6A, B and 7D), other alterations were observed frequently. These were a general reduction in size, an underdeveloped shell field and the presence of large, cell-free spaces in the anterior half of the embryo (Fig. 7). Smaller sized embryos and larvae with an "immature" shell field seem to have developed slower than the control embryos but are otherwise normally developed. In contrast, the embryos in which blister-like, cell-free spaces had formed revealed distinct features. Most of the main larval organs as revealed by endogenous AP activity were present, although less far developed. In all larvae, very small protonephridia, a rudimentary but invaginated shell gland, a stomodeum and ciliated prototrochal and apical plate cells could be observed (Fig. 7A-C, F). By contrast, in all of these embryos the large-vacuolar larval liver cells (Fig. 7A, black arrows) of the archenteron were not differentiated. The cells at the corresponding positions were much smaller and lacked the characteristic large vacuoles (Fig. 8; see also chapter 2, Fig. 2). Furthermore, the lumen of the archenteron was narrow and much smaller. The space usually occupied by the vacuoles of the larval liver cells seems to be filled by fluid (Fig. 7A, B, C and F, marked by x's). The larval liver cells have been shown to be crucial for the embryonic nutrition. The intracapsular fluid that is the main source of nourishment for the embryo is uptaken by pinocytosis and digested in their large vacuoles [26-28]. Therefore, abnormal or missing differentiation of these large-vacuolar larval liver cells is conceivable to result in insufficient nutrition. The regress of larval liver cells has been described as a common malformation resulting from the manipulation of the normal development but was associated with an arrested development at the trochophore stage [17]. Three embryos that exhibited blister-like malformations at $70 \mathrm{hpfc}$ were allowed to develop until $9 \mathrm{dpfc}$, which is close to hatching. Two of these embryos died after five days while the remaining embryo revealed a large, cell-free space in the foot (data not shown). 


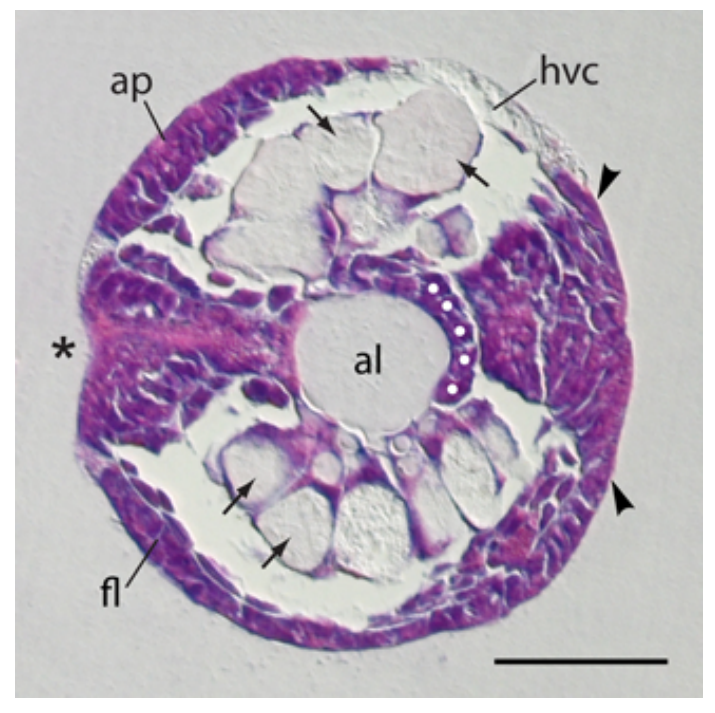

Fig. 8. Cellular characteristics of the archenteron in the undisturbed veliger larva. The archenteron comprises large-vacuolar larval liver cells (black arrows) that occupy most of the internal space in normally developed larvae. The small-celled endoderm that is in contact with the invaginated shell gland (out of section plane) is the most dorsal part of the archenteron (white dots). Sagittal semithin section of a $59 \mathrm{hpfc}$ old veliger larva stained with haematoxylin and eosin. The embryo is oriented with anterior to the top and reflected about the vertical axis for consistency of presentation. An asterisk marks the position of the blastopore. Black arrowheads mark the position of the shell gland. Abbreviations: $\mathrm{ap}=$ apical plate, $\mathrm{fl}=$ foot lobe, $\mathrm{hvc}=$ head vesicle cells, al= lumen of the archenteron. The scale bar is $50 \mu \mathrm{m}$.

The undifferentiated larval liver cells might imply a role for Notch signalling in the formation of the archenteron, in particular the normal cellular differentiation. Notch signalling would then exert this function already during the early cleavage phase (table 1). At least the Notch receptor is known to be maternally provided in the annelid $H$. robusta [19]. In general, molluscan embryos are thought to develop in a mosaic fashion (e.g. $[29,30]$ ), in which cell fates are rather autonomously specified than by cell signalling-dependent induction mechanisms as carried out by the Notch pathway. Direct cell-cell signalling during the early cleavage phase is known to occur during the specification of an embryonic organiser (the 3D macromere) and its subsequent interactions with other cells. The organiser activity of 3D consists of the establishment of dorsoventral polarity by inducing the animal micromeres to their fate to produce ectodermal structures. This leads to the development of a dorsoventral patterning of the embryo (e.g. [31-33]. However, it does not seem likely that inhibition of Notch signalling during the cleavage/blastula phase in $L$. stagnalis interferes with the process of 3D specification or its signalling to the animal micromeres. Firstly, several studies in which the specification and patterning activity of 3D was disturbed report radially organised or dumbbell-shaped larvae [34-36]. Secondly, ectodermal structures such as the apical plate, prototroch and shell field are present and partially well developed. This contrasts with findings in the Caenogastropod Ilyanassa obsoleta [37]. DAPT-mediated inhibition of Notch signalling in $I$. obsoleta results in defects in ectodermal structures such as foot, shell and velum (which corresponds to the differentiated prototroch in many molluscs) and internal organs. The authors do not detail these defects, which impedes further 
comparisons. However, at least the prototroch and the foot appeared normal in $L$. stagnalis. It remains unclear whether the defects in the shell and internal organs in $I$. obsoleta resemble the rudimentary shell gland and undifferentiated larval liver cells in L. stagnalis. Later studies in I. obsoleta report a truncated posterior growth [38], which might be reflected by the relatively small posttrochal ectodermal region in $L$. stagnalis phenotypes (Fig. 6B and F). The authors conclude that Notch signalling might function in regulating posterior growth as part of a gene regulatory network which is conserved among bilaterians. The authors do not state whether the truncated posterior growth was associated with other developmental abnormalities such as the undifferentiated larval liver cells observed in L. stagnalis. It therefore remains speculative whether the DAPT phenotypes in I. obsoleta and L. stagnalis reveal corresponding features and whether the truncated axial growth in I. obsoleta could be interpreted more generally as the result of insufficient nourishment due to malformation of the archenteron.

To date, the molecular basis of the development of the archenteron in molluscan embryos is not known. Similarly, the presence of Notch pathway transcripts or proteins in cleavage and blastula stages of molluscan embryos has not been demonstrated yet.

\section{Conclusions}

My study represents the first investigation into the molecular framework underlying the specification of the molluscan larval shell field. The spatial expression of core Notch pathway components and the pharmacological inhibition of Notch signalling do not support the hypothesis of a Notch-mediated induction mechanism that specifies the shell field in L. stagnalis. Rather, these first observations of Notch signalling in L. stagnalis indicate a potential involvement of the Notch pathway during early neurogenesis. Furthermore, deficiencies in the formation of the archenteron after inhibition of active signalling at the cleavage/blastula stage imply that Notch signalling is active at an early developmental stage. This suggests that the Notch pathway is maternally inherited in L. stagnalis. This study represents the first revisal of Notch signalling during molluscan shell field development whose molecular basis is largely unknown. 


\section{References}

1. Jackson DJ, Wörheide G, Degnan BM: Dynamic expression of ancient and novel molluscan shell genes during ecological transitions. BMC Evol Biol 2007, 7:160.

2. Samadi L, Steiner G: Involvement of Hox genes in shell morphogenesis in the encapsulated development of a top shell gastropod (Gibbula varia L.). Dev Gen Evol 2009, 219:523-530.

3. Raven CP: Morphogenesis in Limnaea stagnalis and its disturbance by lithium. J Exp Zool 1952, 121:1-77.

4. Hohagen J, Jackson D: An ancient process in a modern mollusc: early development of the shell in Lymnaea stagnalis. BMC Dev Biol 2013, 13:27.

5. Kniprath E: Ontogeny of the Molluscan Shell Field: a Review. Zool Script 1981, 10:61-79.

6. Barolo S, Posakony JW: Three habits of highly effective signaling pathways: principles of transcriptional control by developmental cell signaling. Genes Dev 2002, 16:1167-1181.

7. Gazave E, Lapébie P, Richards GS, Brunet F, Ereskovsky AV, Degnan BM, Borchiellini C, Vervoort M, Renard E: Origin and evolution of the Notch signalling pathway: an overview from eukaryotic genomes. $\mathrm{BMC} \mathrm{Evol} \mathrm{Biol}$ 2009, 9:249.

8. Kimble J, Simpson P: The LIN-12/Notch signaling pathway and its regulation. Ann Rev Cell Dev Biol 1997, 13:333-361.

9. Bray S: Notch signalling in Drosophila: three ways to use a pathway. Semin Cell Dev Biol 1998, 9:591-597.

10. Bray SJ: Notch signalling: a simple pathway becomes complex. Nat Rev Mol Cell Biol 2006, 7:678-689.

11. Castro B, Barolo S, Bailey AM, Posakony JW: Lateral inhibition in proneural clusters: cis-regulatory logic and default repression by Suppressor of Hairless. Development 2005, 132:3333-3344.

12. Heitzler $P$, Simpson $P$ : The choice of cell fate in the epidermis of Drosophila. Cell 1991, 64:1083-1092.

13. D'Souza B, Miyamoto A, Weinmaster G: The many facets of Notch ligands. Oncogene 2008, 27:5148-5167.

14. Fortini ME: Notch Signaling: The Core Pathway and Its Posttranslational Regulation. Dev Cell 2009, 16:633-647. 
15. Bailey A, Posakony J: Suppressor of Hairless directly activates transcription of Enhancer of split complex genes in response to Notch receptor activity. Genes Dev 1995, 9:2609-2622.

16. Thomas JD, Lough AS, Lodge RW: The Chemical Ecology of Biomphalaria glabrata (Say), the Snail Host of Schistosoma mansoni Sambon: The Search for Factors in Media Conditioned by Snails which Inhibit their Growth and Reproduction. J Appl Ecol 1975, 12:421-436.

17. Morrill JB: Development of the Pulmonate Gastropod, Lymnaea. In Developmental Biology of Freshwater Invertebrates. Edited by Harrison FW, Cowden RR. New York: Alan R. Liss, Inc.; 1982:399-483.

18. Kocot KM, Cannon JT, Todt C, Citarella MR, Kohn AB, Meyer A, Santos SR, Schander C, Moroz LL, Lieb B, Halanych KM: Phylogenomics reveals deep molluscan relationships. Nature 2011, 477:452-456.

19. Gonsalves F, Weisblat D: MAPK regulation of maternal and zygotic Notch transcript stability in early development. Proc Natl Acad Sci U S A 2007, 104:531.

20. Hejnol A, Martindale MQ, Henry JQ: High-resolution fate map of the snail Crepidula fornicata: The origins of ciliary bands, nervous system, and muscular elements. Dev Biol 2007, 305:63-76.

21. Lai E: Notch signaling: control of cell communication and cell fate. Development 2004, 131:965-973.

22. Kageyama R, Ohtsuka $T$, Kobayashi $T$ : The Hes gene family: repressors and oscillators that orchestrate embryogenesis. Development 2007, 134:12431251.

23. Voronezhskaya EE, Hiripi L, Elekes K, Croll RP: Development of Catecholaminergic Neurons in the Pond Snail, Lymnaea stagnalis: I. Embryonic Development of Dopamine-Containing Neurons and Dopamine-Dependent Behaviors. J Comp Neurol 1999, 404:285-296.

24. Voronezhskaya E: Apical sensory neurones mediate developmental retardation induced by conspecific environmental stimuli in freshwater pulmonate snails. Development 2004, 131:3671-3680.

25. Serras F, Notenboom R, Van den Biggelaar J: Communication Compartments in the Post-trochal Ectoderm of the Mollusc Lymnaea stagnalis. Exp Cell Res 1990, 187:284.

26. Beadle L: Salt and water regulation in the embryos of freshwater pulmonate molluscs I. The embryonic environment of Biomphalaria 
sudanica and Lymnaea stagnalis. J Exp Biol 1969, 50:473-479.

27. Arni P: Licht-und elektronenmikroskopische Untersuchungen an Embryonen von Lymnaea stagnalis L. (Gastropoda, Pulmonata) mit besonderer Berücksichtigung der frühembryonalen Ernährung. Zoomorphology 1974, 78:299-323.

28. Arni P: Licht-und elektronenmikroskopische Untersuchungen zur Entwicklung und Degeneration transitorischer Speicherzellen der Mitteldarmregion von Lymnaea stagnalis L. (Gastropoda, Pulmonata). Zoomorphology 1975, 81:221-240.

29. Serras F, Van den Biggelaar J: Is a mosaic embryo also a mosaic of communication compartments? Dev Biol 1987, 120:132-138.

30. Lambert J, Nagy L: MAPK signaling by the D quadrant embryonic organizer of the mollusc Ilyanassa obsoleta. Development 2001, 128:45-56.

31. Lambert J, Nagy L: The MAPK cascade in equally cleaving spiralian embryos. Dev Biol 2003, 263:231-241.

32. van den Biggelaar JAM, Dictus WJAG: Gastrulation in the Molluscan Embryo. In Gastrulation - From Cells to Embryo. Edited by Stern CD. New York: Cold Spring Harbor Laboratory Press; 2004:63-78.

33. Lambert J: Mesoderm in spiralians: the organizer and the $4 \mathrm{~d}$ cell. J Exp Zool 2008, 310B:15-23.

34. Martindale MQ, Doe CQ, Morrill JB: The role of animal-vegetal interactions with respect to the determination of dorsoventral polarity in the equalcleaving spiralian, Lymnaea palustris. Dev Gen Evol 1985, 194:281-295.

35. Gonzales E, Zee M, Dictus W, Biggelaar J: Brefeldin A or monensin inhibits the 3D organizer in gastropod, polyplacophoran, and scaphopod molluscs. Dev Gen Evol 2007, 217:105-118.

36. Koop D, Richards G, Wanninger A, Gunter H, Degnan B: The role of MAPK signaling in patterning and establishing axial symmetry in the gastropod Haliotis asinina. Dev Biol 2007, 311:200-212.

37. Nagy LM, Gharbiah M, Nakamoto A, Wandelt J: Evidence for multiple signaling events during the early cleavages of the mollusc Ilyanassa [abstract]. Dev Biol 2009, 331:401.

38. Nakamoto A, Harrison C, Gharbiah M, Nagy LM: Notch and Wnt signaling in axial elongation in the mollusc embryo Ilyanassa obsoleta [abstract]. Dev Biol 2010, 344:529-530. 


\section{General discussion}

More than 545 million years ago, early molluscan life forms acquired the ability to produce biomineralised shells. Today, molluscan shells reveal an incredible diversity of form, structure and ornamentation and are strongly adapted for various ecological niches. Arguably, the evolutionary success of the Mollusca was facilitated by the innovation of the shell. The fossil record conveys the timing of its evolutionary emergence but inferences about how the shell might have been originated must derive from the study of shell development in recent molluscs. Many ontogenetic events associated with the initiation of shell formation in the embryo are evolutionarily conserved (reviewed in [1]; p. 6). In particular, the initial specification of the future shell forming cells is often accompanied by direct cellular interactions: the shell field progenitor cells are in contact with underlying endodermal cells in disparate molluscan classes (Fig. 1). This contact was proposed to mediate an induction-dependent specification of the future shell forming cells [2]. Until now, this hypothesis remains the canonical theory of molluscan shell field specification.

The deeply conserved morphogenesis of the larval shell field suggests that the underlying molecular program might be similarly conserved (pp. 9). In my thesis, I evaluated the role of the contact-dependent Notch signalling pathway in the specification of the shell field in the derived gastropod Lymnaea stagnalis. This represents the first study into the molecular mechanisms that specify the molluscan shell field. I first characterised the development of the shell field in L. stagnalis using a combination of light and electron microscopy techniques (chapter 2). This study describes the exact timing of developmental events that are critically related to the specification process (the initial formation of the contact and the first visible cellular differentiation) and thus allowed to determine the time point of the shell field specification in L. stagnalis. After optimising a whole mount in situ hybridisation method for this non-model organism (chapter 3), particularly this time point was further investigated. Precisely, the expression of Notch pathway orthologues in L. stagnalis was examined and active Notch signalling was pharmacologically inhibited during the presumably specifying contact event (chapter 4). 
In L. stagnalis, the cellular arrangements and differentiation events during the contact formation between the future shell forming cells and endodermal cells of the underlying archenteron are in congruence with a specification via contact-mediated induction. The first visible cellular differentiation ensues after this contact is formed (chapter 2, Fig. 2; see also pp. 32). However, conflicting findings have been reported for many molluscan species. In fact, morphogenic conditions that are in agreement with a contact-mediated shell field induction were as frequently observed as those contradicting it (reviewed in [1]; see chapter 2, pp.41). No tendency towards a contact-associated or contact-independent shell field specification mechanism is evident within the Mollusca: both modes of early shell field development are almost equally realised in all major lineages (Fig. 1).

A number of previous studies documents the development of experimentally manipulated embryos [3-6]. In these studies, cell deletion or blastomere separation experiments were performed during the early cleavage phase. Potentially, this allows to discriminate between inductive and cell-autonomous specification events (in which the cell fate is inherently determined; see p. 7). The results of these studies favour an induction-dependent mode of shell field specification, but disagree about the timing and the participating cells/tissues (see pp. 41 for a detailed discussion).

To my knowledge, the only alternative explanation for the widespread contact event was suggested by Kniprath [7]. He interpreted the contact formation as prerequisite for the later shell field invagination. Herein, the primary role of this contact would be the mechanical fixation of the proliferating shell field cells to the archenteron. The increasing number of cells within a stably sized shell field epithel would force the shell field epithel to evade inwards, thus to invaginate. Kniprath interprets the presence of intercellular connective structures (gap junctions) between the participating epithels as supportive evidence for this scenario, and further concludes that the final evagination of the shell gland represents a delayed retention. However, a function of the contact in creating adhesive force does not exclude a parallel role in the transmission of an inductive signal that specifies the molluscan shell field.

There is no evidence that Notch signalling exerts this function in L. stagnalis. None of the retrieved Notch pathway orthologues was expressed in the (prospective) shell field or the presumably inducing endodermal cells (chapter 4, Figs. 3 and 4). Also, the pharmacological inhibition of Notch signalling during the contact formation had no effect on the shell field appearance (pp. 89). 


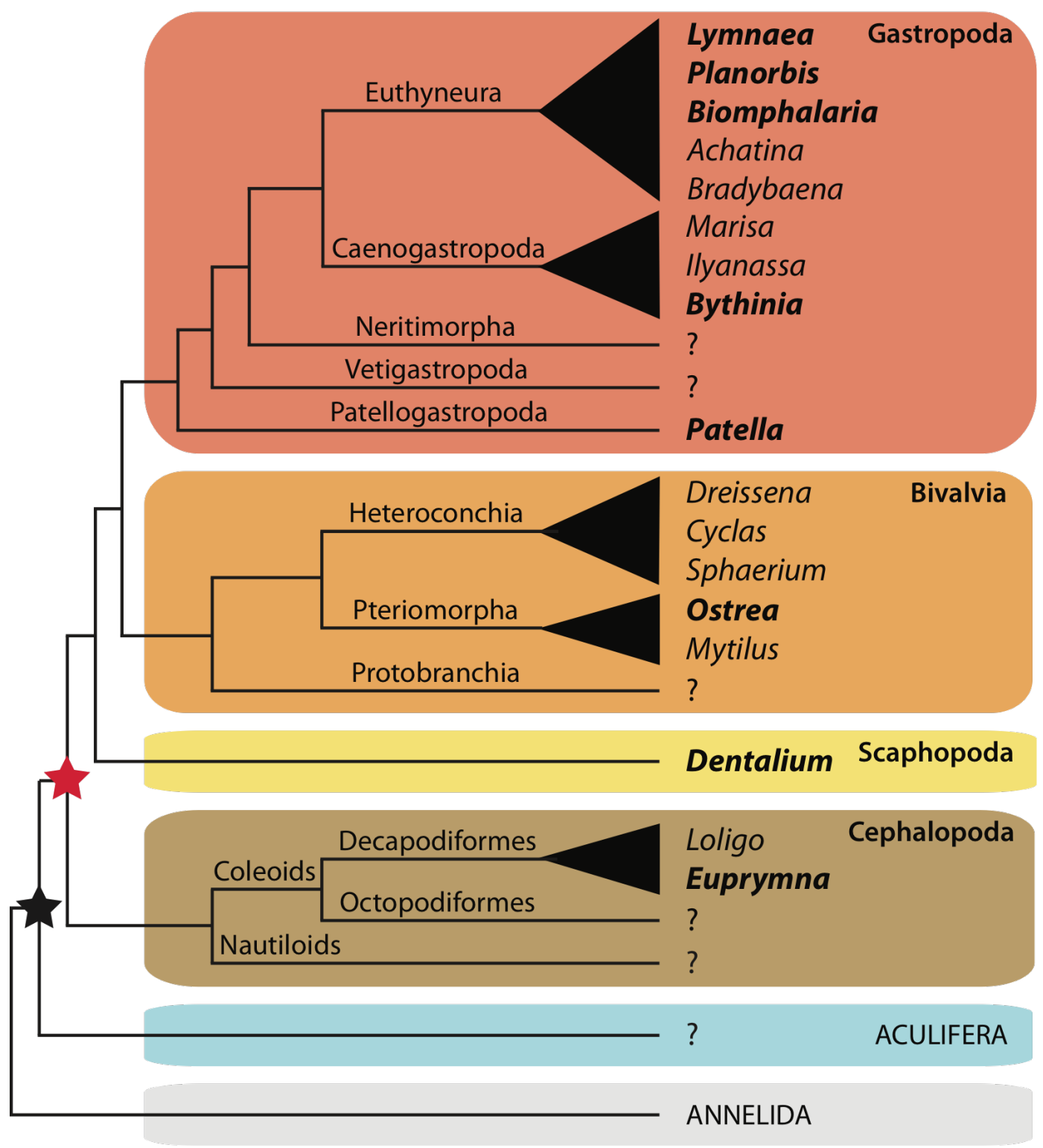

Fig. 1. Morphogenic conditions that favour a contact mediated shell field induction are widespread within the Mollusca (taxa in bold). Comparative studies will allow to infer the gene regulatory network underlying conchiferan shell development (red asterisk) and to deduce its ancestral state within the Mollusca (black asterisk). Topology adapted from [8].

Only larvae that were manipulated well before the shell field is presumably specified frequently developed a rudimentary shell gland (chapter 4, Figs. 5 and 6). However, this seems to rather reflect unspecific effects of an overall disturbed development than to reveal specific interference with the shell forming program (pp. 97). The expression of the Notch ligand delta in the gastropod Haliotis asinina but not in L. stagnalis contrasts with the deep conservation in the spatial expression of other shell field-associated genes (pp. 11). The delta orthologue in H. asinina 
reveals the typical structure of canonical DSL ligands (other "non-canonical" Notch ligands exists whose mode of operation is less well understood, reviewed in e.g. [9]) and is thus likely to indicate canonical Notch signalling. In L. stagnalis, the presence of unknown orthologues of canonical Notch receptors and ligands cannot be excluded in the absence of an annotated genome. Though, the number of retrieved $d s l$ genes is high in relation to the number of paralogues present in other metazoans and corresponds to the number of DSL genes in the only other studied mollusc, Lottia gigantea [10].

Expectably, the expression of a genetic subset that specifies the progenitor field of a homologous structure is evolutionarily conserved ([11, 12]; see also pp. 9). A flexible deployment of the Notch pathway during the shell field specification in H. asinina and L. stagnalis defies this expectation. This raises the question of the ancestral genetic conditions under which the shell first developed. If these two species employ different molecular programs in shell formation, their shells and the similarities in conchiferan shell development would be the result of convergent evolution. Thus, the conchiferan shell would have multiple evolutionary origins. Alternatively, an ancestral shell forming gene regulatory network evolved once at the base of the Conchifera and since then experienced secondary modifications.

In both cases, a variable deployment of Notch signalling in $H$. asinina and L. stagnalis possibly affects downstream processes, which might be reflected by variations in their shell field morphogenesis. Such differences are potentially highly informative: it might be possible to associate unique developmental features with the action of variably employed genes. Unfortunately, the course of the shell field morphogenesis in $H$. asinina is not yet described with sufficient spatial and temporal resolution, permitting such comparisons and exemplifying the need for more elaborate molluscan model organisms.

The identification of the specifying genes will contribute to the reconstruction of the gene regulatory network that governs shell development in L. stagnalis. Knowledge on the shell forming gene regulatory network in one molluscan representative will provide the ground for comparative studies within and beyond the conchiferan molluscs. Such comparative studies bear the potential to shed light on various unresolved aspects of molluscan shell evolution (Fig. 1), ranging from the generation of phenotypic diversity to the homology of the biomineralising tissues in conchiferan and aculiferan molluscs. Comparing how the shell field specifying genes 
are deployed in early branching conchiferans, the plated polyplacophorans and the non-shelled aculiferan clades will allow to infer the structure of the ancestral shell forming gene regulatory network and thus the conditions under which the molluscan shell first arose.

\section{Outlook}

The identification of the molecular regulators of shell field specification represents an important step towards a deeper understanding of how the molluscan shell first developed. The present lack of molluscan model organisms precludes the use of techniques such as systematic gene knockdown commonly employed in derived genetic model systems. Currently, investigations into the molecular basis for a given process in Lymnaea and other emerging molluscan model systems still rely on candidate genes. Digital gene expression profiling represents a powerful technique to identify candidate genes and genetic pathways. For instance, comparing the transcriptome of different ontogenetic stages might highlight genes that are upregulated in accordance with particular developmental events. In terms of shell field specification in L. stagnalis, transcriptome data from developmental stages before, during and after the presumably specifying contact is formed is likely to identify differentially expressed genes whose spatial expression and functions can subsequently be explored. 


\section{References}

1. Kniprath E: Ontogeny of the Molluscan Shell Field: a Review. Zool Script 1981, 10:61-79.

2. Raven CP: Morphogenesis in Limnaea stagnalis and its disturbance by lithium. J Exp Zool 1952, 121:1-77.

3. Hess O: Die Entwicklung von Exogastrulakeimen bei dem SüsswasserProsobranchier Bithynia tentaculata L. Dev Gen Evol 1956, 148:474-488.

4. Hess O: Die Entwicklung von Halbkeimen bei dem Süsswasserpulmonaten Limnaea stagnalis L. Dev Gen Evol 1957, 150:124-145.

5. Cather JN: Cellular Interactions in the Development of the Shell Gland of the Gastropod, Ilyanassa. J Exp Zool 1967, 166:205-223.

6. Labordus V, van der Wal UP: The determination of the shell field cells during the first hour in the sixth cleavage cycle of eggs of Ilyanassa obsoleta. $J$ Exp Zool 1986, 239:65-75.

7. Kniprath E: Zur Ontogenese des Schalenfeldes von Lymnaea stagnalis. Dev Gen Evol 1977, 181:11-30.

8. Kocot KM, Cannon JT, Todt C, Citarella MR, Kohn AB, Meyer A, Santos SR, Schander C, Moroz LL, Lieb B, Halanych KM: Phylogenomics reveals deep molluscan relationships. Nature 2011, 477:452-456.

9. D'Souza B, Miyamoto A, Weinmaster G: The many facets of Notch ligands. Oncogene 2008, 27:5148-5167.

10. Gazave E, Lapébie P, Richards GS, Brunet F, Ereskovsky AV, Degnan BM, Borchiellini C, Vervoort M, Renard E: Origin and evolution of the Notch signalling pathway: an overview from eukaryotic genomes. BMC Evol Biol 2009, 9:249.

11. Wagner G: The developmental genetics of homology. Nat Rev Gen 2007, 8:473479.

12. Erwin $\mathrm{DH}$, Davidson $\mathrm{EH}$ : The evolution of hierarchical gene regulatory networks. Nat Rev Genet 2009, 10:141-148. 


\section{Acknowledgements}

I am very grateful to my supervisor Daniel Jackson for giving me the opportunity to work in a research field that constantly fascinates and excites me, for sharing his knowledge and enthusiasm with me, and for the many critical and constructive discussions. Thank you for teaching me.

I also would like to thank Joachim Reitner as well as Volker Thiel, Gregor Bucher, Frank Melzner and Frédéric Marin for co-supervision or participation in my thesis committee. I gratefully acknowledge Wolfgang Dröse and Dorothea Hause-Reitner for expert technical assistance in histological and scanning electron microscopy experiments, and Luciana Macis for not only maintaining the snail cultures but also caring for the numerous details that keep a lab running.

I am indebted to my former and present colleagues who provided an inspiring work atmosphere. Sincere thanks are given to Ines Herlitze, Juliane Germer, Leyla Seyfullah, Christine Heim, Mahesh Desai, Sharmishta Dattagupta, Diana and Jan Bauermeister and Tim Leefmann. I am also deeply indebted to Christina Beimforde and Nora Glaubrecht. Thank you for your enduring support and commitment. Last but not least, I would like to thank my family and friends most sincerely for their everlasting encouragement and support in every aspect. 
Appendices 


\section{Appendix 1}

\section{Validation of internal reference genes for quantitative real time reverse transcription PCR in Lymnaea stagnalis}

\section{Introduction}

Gene expression profiling by quantitative real time reverse transcription PCR (qRTPCR) is a powerful approach to detect gene transcripts and quantify their abundance. The design of qRT-PCR experiments can be adapted to test hypotheses about gene regulation in any biological context. In developmental biology, qRT-PCR is commonly employed to detect differentially expressed genes during an ontogenetic process or to confirm gene knock-down experiments. This technique allows for the quantification of a wide range of expression levels from small amounts of tissue and is capable to detect even minor changes in gene expression across different tissues or developmental stages. Also, it enables the simultaneous and expeditious analysis of multiple genes. Therefore, qRT-PCR is particularly useful for investigating processes for which there is less previous knowledge about the underlying gene regulatory network like the molluscan shell field specification.

Conceptually, qRT-PCR involves the following steps: the extraction of RNA from the samples to compare, the reverse transcription into cDNA, the determination of the amplification behaviour of the studied gene using fluorescent dyes or probes and the quantification of the resulting signal. However, to ensure comparability of individual results, qRT-PCR studies have to account for several variables.

Differences in the amount and overall transcriptional activity of the starting material and varying efficiencies of enzymatic reactions (e.g. [1, 2]) necessitate the normalisation of qRT-PCR data across samples. Common normalisation strategies are the standardisation against the number of cells, the total RNA mass, the fraction of ribosomal RNA, externally added RNA standards or the expression levels of reference genes [1, 2]. However, the accurate quantification of the total cell number and RNA mass of the starting material is not always possible. Furthermore, normalisation against the total RNA mass does not account for variations in RNA quality and enzymatic efficiencies, and the relationship between rRNA and mRNA has been shown to vary [1]. Therefore, the most frequently employed normalisation 
strategy is the use of internal reference genes that display stable expression levels in all samples under investigation. Commonly employed reference genes are housekeeping genes whose expression is required for the basic cell survival (e.g. [1-3]) and hence is assumed to remain constant under varying developmental and environmental conditions. However, most studies explicitly testing this assumption report considerable variations in housekeeping gene expression, highlighting the need for the prior validation of the uniform expression of potential reference genes $[1,3-6]$.

Another important factor in qRT-PCR experiments is the amplification efficiency, which directly affects the credibility of qRT-PCR results. Similar amplication efficiencies of compared samples are required for reliably relating their expression levels to each other, although mathematical models can correct for minor differences in the amplification efficiencies (reviewed in [2]). Highly efficient reactions take place near the optimum, vary less and are reliably reproducible [7]. Since PCR efficiencies are usually assessed beforehand using standard curves, and only one reaction is repeated in the final experiment, this reaction needs display the priorly determined efficiency. Lowly efficient reactions might not perform identically when repeated in the final experiments, which will impede the reliable comparison of different reactions, thus samples.

The establishment of a validated set of reference genes for qRT-PCR based gene expression profiling in L. stagnalis will facilitate molecular studies on a wide range of developmental processes including the shell field specification. Here I report preliminary results on the evaluation of several candidate reference genes with putatively constant expression levels in L. stagnalis.

\section{Materials and Methods}

\section{Selection of candidate reference genes}

Eighteen previously used candidate genes were initially selected from literature for gene expression studies in other molluscan species or the validation of reference genes. The chosen genes were selected to represent a wide range of presumptive expression levels and different functional classes to minimise possible co-regulation $[1,3]$. Their sequences were retrieved from GenBank for tBLASTn searches against two larval and one adult tissue transcriptome database of L. stagnalis. The most 
similar ESTs for each library were chosen for subsequent BLAST searches against a draft genomic database of L. stagnalis to identify the putative orthologues of the literature candidates in L. stagnalis. If the ESTs from at least two libraries could be allocated to the same genomic contig, this locus was considered to represent the orthologue in L. stagnalis. For five literature-derived candidates, no putative orthologues in L. stagnalis were identified in the available L. stagnalis databases and were therefore discarded. An overview about the remaining thirteen candidate genes is provided in table 1 .

\section{Primer design and verification}

Initially, the retrieved ESTs and genomic contigs were assembled to identify introns and annotate splice sites. Primers were manually designed to span exon boundaries or to allocate to different exons, which allows to discriminate against amplification of genomic contamination [2,3]. These primer were subsequently analysed using the primer analysis software Primer 3 (http://bioinfo.ut.ee/primer3-0.4.0/) and OligoAnalyzer 3.1 (http://eu.idtdna.com/analyzer/Applications/OligoAnalyzer/) and modified to minimise the predicted formation of secondary structures. All primers were designed to reveal similar melting temperatures. If possible, several primers were designed to amplify different fragments ranging from 105 to 332 base pairs length. The predicted introns of each candidate gene were confirmed by amplification of genomic DNA using conventional PCR. All primer sequences are provided in table 2 .

\section{RNA extraction}

Total RNA was extracted from four different adult tissues and four distinct larval stages selected to represent major phases of embryonic development. The adult tissues were derived from the mantle, the head, the foot and the heart. Investigated developmental stages were pooled cleavage and gastrulation, trochophore larva ( $\sim 57$ hours post first cleavage), early veliger larva ( $\sim 97$ hours post first cleavage) and late (shelled) veliger larva. Adult total RNA was extracted using the TRI Reagent (Ambion, \#AM9738) with an optional high salt precipitation step. Embryonic total RNA was extracted using the QIAgen Rneasy Plus Micro Kit (Qiagen, \#74034), according to the manufacturer's instructions. Total RNA from pooled cleavage and gastrulation stages and the trochophore stage were subsequently purified and concentrated by precipitation using sodium acetate and ethanol. All RNA extractions 
were quantified by three independent nanodrop measurements and calculation of the average concentration value. From each adult RNA extraction, 500 ng were denatured in $90 \%$ de-ionised formamide at $85^{\circ} \mathrm{C}$ for ten minutes and were qualitatively assessed by agarose gel electrophoresis. The overall yield of the embryonic RNA extractions was too low for agarose gel electrophoresis.

\section{Reverse transcription}

Total RNA was reverse transcribed using Oligo dT primer into cDNA representing the polyadenylated mRNA fraction. Reverse transcription was performed on $500 \mathrm{ng}$ total RNA as described in chapter 4. Additionally, both Oligo dT and random hexamer primed cDNA libraries from $1 \mu \mathrm{g}$ adult head RNA were synthesised using the same protocol.

\section{Optimisation of PCR parameters}

Unspecific amplification and the formation of primer dimerisation products reduce the amplification efficiency. This compromises the accuracy and reproducibility of qRT-PCR experiments and renders an initial optimisation of each reaction necessary. The thermoprofile and primer concentrations for each amplicon were optimised using conventional PCR on cDNA and genomic DNA. In all experiments, no template controls (water) and no reverse transcription controls (RNA diluted to the presumptive remaining concentration in the cDNA) were included. The PCR parameters that generated no amplification of genomic contaminations (recognisable by a larger amplicon size in cDNA or amplification of genomic DNA or RNA) or primer dimerisation were further used. Initial qRT-PCR experiments on both CDNA and genomic DNA were carried out to adapt the optimised PCR parameters for real time conditions and to test different qRT-PCR reagents. All further qRT-PCR reactions were performed using SYBR Green (SensiFAST SYBR No-ROX kit; Bioline, \#BIO98005) on a Rotor-Gene Q 3000 (Qiagen) in $15 \mu \mathrm{L}$ volumes. All subsequent reactions were carried out in duplicates or triplicates. A final melt curve was always included in the thermoprofile to prove for unspecific amplification and primer dimerisation. No template controls and no reverse transcription controls were always included.

\section{Assessment of PCR efficiency}

The PCR efficiencies for each one amplicon of most candidate genes were evaluated on pooled cDNA and several distinct cDNA libraries (pooled cleavage and 
gastrulation, early veliger larva and adult head). Several 5-point dilution series were used to construct calibration curves, namely 2-fold, 4-fold, 10-fold and 20-fold. Additionally, both the effects of different RNA amounts and priming strategies for reverse transcription were tested by the example of adult head tissue. The amplification efficiencies were calculated from the slopes of the calibration curves using the equation $10^{-(1 / \text { slope })}-1$ by the Rotor Gene software.

\section{Preliminary results and discussion}

The initial amplification efficiency was for all tested candidate gene amplicons except for GAPDH below 0.9, suggesting an inhibition of the PCR reaction that equally affects the amplification of all genes. Expanding the dilution range of the standard curve and excluding the lower dilutions increased the efficiency for most candidate genes (GAPDH, NACA, $\alpha$-act, EF1 $\alpha, R P L 13 \alpha$ ), corroborating the putative presence of an inhibitory substance. However, for some candidate genes $(E F 1 \alpha$, $R P L 13 \alpha$ ), reactions using pooled cDNA at dilutions larger than 1:1000 frequently failed in some of the replicates. This indicates that the range of template concentration in which amplification is predictable (the dynamic range) was exceeded. Although it was possible to determine for most genes a dilution range in which amplification took place with a efficiency around 1.0, this dilution range might not allow to efficiently amplify lowly expressed genes. Reference gene candidates with low expression levels are of particular interest for developmental gene expression profiling. Ideally, internal reference and target genes display similar "crossing points" (which corresponds to the transcript abundance under efficient PCR conditions)[1, 2]. However, developmentally regulated transcription factors and signalling molecules might be lowly abundant. Therefore, the dynamic range for efficient amplification needs to be expanded to include undiluted or lowly diluted templates with higher transcript numbers. This can be done by either removal or attenuation of the inhibitory influence or by increasing the efficiency of the reverse transcription.

To prove, whether the presence of the putative inhibitory substance can be related to different RNA preparation methods, distinct libraries were directly compared to pooled cDNAs. First, the pooled cleavage and gastrulation sample that has been 
Table 1. Reference gene candidates for L. stagnalis.

* This candidate gene has been recommended by Primerdesign

(www.primerdesign.co.uk/geNorm\%20plus\%20kits.html; November $8^{\text {th }} 2011$ ).

\begin{tabular}{|c|c|c|c|}
\hline Gene name & Abbreviation & Biological function & $\begin{array}{l}\text { Reference reporting this } \\
\text { gene as suitable qPCR } \\
\text { reference gene }\end{array}$ \\
\hline$\alpha$-tubulin (contig1) & $\alpha-t u b 1$ & $\begin{array}{l}\text { cytoskeletal structural } \\
\text { protein }\end{array}$ & {$[3,5,8]$} \\
\hline$\alpha$-tubulin (contig2) & $\alpha-t u b 2$ & $\begin{array}{l}\text { cytoskeletal structural } \\
\text { protein }\end{array}$ & {$[3,5,8]$} \\
\hline ribosomal protein $L 13 \alpha$ & $R P L 13 \alpha$ & $\begin{array}{l}\text { structural component of } \\
\text { the } 60 \mathrm{~S} \text { ribosomal subunit }\end{array}$ & {$[1,3]$} \\
\hline $\begin{array}{l}\text { RNA polymerase II } \\
(R N A P) \text { subunit } R P B 4\end{array}$ & $R N A P$ & enzyme for transcription & [3] \\
\hline TATA box binding protein & $T B P$ & $\begin{array}{l}\text { RNA polymerase II } \\
\text { transcription factor }\end{array}$ & {$[1,3,9]$} \\
\hline elongation factor I $\alpha$ & $E F 1 \alpha$ & $\begin{array}{l}\text { factor for protein } \\
\text { translation }\end{array}$ & {$[3,5,10,11]$} \\
\hline $\begin{array}{l}\text { nascent polypeptide- } \\
\text { associated complex } \alpha- \\
\text { polypeptide }\end{array}$ & $N A C A$ & $\begin{array}{l}\text { protein associated with } \\
\text { protein translation }\end{array}$ & [12] \\
\hline $\begin{array}{l}\text { ubiquitin-conjugating } \\
\text { enzyme E2D } 2\end{array}$ & $U B E 2 D 2$ & $\begin{array}{l}\text { enzyme involved in } \\
\text { protein degradation }\end{array}$ & $*$ \\
\hline $\begin{array}{l}\text { Succinate dehydrogenase } \\
\text { complex, subunit A }\end{array}$ & $S D H A$ & $\begin{array}{l}\text { enzyme in mitochondrial } \\
\text { respiratory chain }\end{array}$ & {$[1,3]$} \\
\hline $\begin{array}{l}\text { Glyceraldehyde-3- } \\
\text { phosphate } \\
\text { dehydrogenase }\end{array}$ & $G A P D H$ & $\begin{array}{l}\text { enzyme in carbohydrate } \\
\text { metabolism }\end{array}$ & {$[1,3,6]$} \\
\hline $\begin{array}{l}\text { tyrosine 3- } \\
\text { monooxygenase }\end{array}$ & $Y W H A Z$ & $\begin{array}{l}\text { enzyme involved in signal } \\
\text { transduction by binding to } \\
\text { phosphoserine-containing } \\
\text { proteins }\end{array}$ & [3] \\
\hline histon 1 & $H 1$ & chromatin component & [13] \\
\hline $\begin{array}{l}\text { hypoxanthine } \\
\text { phosphoribosyl- } \\
\text { transferase } 1\end{array}$ & $H P R T$ & $\begin{array}{l}\text { enzyme in the purine } \\
\text { salvage pathway }\end{array}$ & {$[1,3,6,9]$} \\
\hline
\end{tabular}

concentrated by ethanol-based precipitation was tested in terms of GAPDH amplification. Second, the early veliger cDNA library that was generated from RNA extracted using a kit was tested in terms of NACA amplification. In both cases, the amplification efficiencies were almost identical. 
Table 2. Primer sequences for all reference gene candidates. * indicates primer that bind to splice sites, ${ }^{+}$indicates primer combinations that span an intron.

\begin{tabular}{|c|c|c|c|c|}
\hline Gene & Forward primer ( 5 ' to $3^{\prime}$ ) & Reverse primer (5' to $3^{\prime}$ ) & $\begin{array}{l}\text { Primer } \\
\text { combination }\end{array}$ & $\begin{array}{l}\text { Amplicon size } \\
\text { (bp) }\end{array}$ \\
\hline \multirow{3}{*}{$\alpha-t u b 1$} & (F1) GCCTCCCTCAGGTTTGATGG* & (R1) CAACTGAAAGCTGCTCATGGTAGG & F1xR1 & 148 \\
\hline & (F2) ATTCATTTCCCCCTGGCTAC & (R2) ATGGTCCTCTTGGTCTTGATGGTA & F1xR2 & 305 \\
\hline & & (R3) CTGATTGGCTGGTTCAAAGC & $\mathrm{F}^{2} \times \mathrm{xR}^{+}$ & 112 \\
\hline \multirow{3}{*}{$\alpha-t u b 2$} & (F1) CTGGCTTCAAGGTTGGCATCA* & (R1) GCCTCAGCAATAGCAGTTGTGTT & F1xR1 & 118 \\
\hline & (F2) AGGTGATGTTGTCCCCAAGG & (R2) TCGACACCAACTTCTTCGTAATCT & $\mathrm{F} 1 \mathrm{xR} 2$ & 271 \\
\hline & & (R3) TGGTGGCTGGTAGTTGATGC & $\mathrm{F}^{2 \times R 3^{+}}{ }^{+}$ & 121 \\
\hline \multirow{2}{*}{$R P L 13 \alpha$} & (F1) TTACAGGAACAAGCTGAAGTTCTTGAAG & (R1) GGCAGTTTTGTGAGGCAACATTC & F1xR1 & 139 \\
\hline & (F2) TTCCATCCAACCTCGTGTGA & (R2) GACCTTTCCATTTCCGTGCT & $\mathrm{F} 2 \times \mathrm{xR}^{+}$ & 238 \\
\hline$R N A P$ & (F1) CCACAACAATCGGGCTCA & (R1) GCTCCTCTTCAGCACTTTCATTC & F1xR1+ & 163 \\
\hline$T B P$ & (F1) CAGGTGCAAAAAGTGAAGACCA & (R1) AAACTGTGCATGTGTAAGGACCAA & F1xR1 ${ }^{+}$ & 176 \\
\hline \multirow{3}{*}{$E F 1 \alpha$} & (F1) GGTGACAATGTTGGTTTCAATGTCAAG & (R1) AAAATGATGACCTGAGCATGGAACTG* & F1xR1 & 128 \\
\hline & (F2) AGTTCCATGCTCAGGTCATCAT* & (R2) CAGACTTAATTACTCCGACAGCAACA & F2xR2 & 306 \\
\hline & (F3) ACGTGTCCGTCAAGGAAATC & (R3) AGTGTGGCAATCAAGCACAG & $\mathrm{F}^{3} \times 3^{+}+$ & 158 \\
\hline \multirow{3}{*}{$N A C A$} & (F1) CAACAAGCACAAGTTGCAGCAG & (R1) AGTCTCATCAATCTCCTCTTCCTCC* & F1xR1 & 137 \\
\hline & (F2) GTTTGAAGCCAGTTCCTGGTG & (R2) ATGTATCAGATGCTGGGCTTTT & F2xR1 & 283 \\
\hline & (F3) TTCCAGAAGAGCTTGTGTCAAA & & $\mathrm{F}^{3} \times 3^{+}+$ & 183 \\
\hline \multirow{3}{*}{ UBE2D2 } & (F1) TGTGGGAGATGATTTATTTCAATGGCA* & (R1) TGGGGGTTTGAATGGGTAGTC & F1xR1 & 122 \\
\hline & (F2) GCTGGACCTGTGGGAGATG & (R2) TGAGGAGTGAGCAGATGGACA & F1xR2 & 258 \\
\hline & & & $\mathrm{F} 2 \times \mathrm{xR}^{+}$ & 130 \\
\hline$S D H A$ & (F1) GCGTCAATGGCAAGCGTATC & (R1) GCACCAACAACCACAGCATC & F1xR1 ${ }^{+}$ & 206 \\
\hline \multirow{2}{*}{ GAPDH } & (F1) GCCCCGCTGGTTAAGGTTAT & (R1) ACGCCAGTCCTTGTTGCTT* & F1xR1 & 120 \\
\hline & (F2) AGGGGCAGACTATGTGGTTGAA & & F2xR1 & 332 \\
\hline \multirow{3}{*}{ YWHAZ } & (F1) AGGAATGAACCAGATGAAGCCTG* & (R1) CAGATGTCCACAGTGTAAGATTATCCCT & F1xR1 & 148 \\
\hline & (F2) ATGCTTGTTTCGCCAACCTG & (R2) CAATGACGTTTTGGAATTGCTGTCC* & F2xR2 & 319 \\
\hline & (F3) ATCCAGAAAGCCCAAGACGA & (R3) CACAGAAAAGTTCAATGCCAATCC & $\mathrm{F}_{3 \times R 3^{+}}^{+}$ & 225 \\
\hline$H 1$ & (F1) CAAAAATGGAAGAAACCGATACTGT & (R1) GAATGGCTTGACGGGATGAG & F1xR1 ${ }^{+}$ & 192 \\
\hline \multirow{2}{*}{ HPRT } & (F1) CGGCAATATCGAAAACCGAAA & (R1) TCCATCAGCAATCCGAATAAAAGA* & F1xR1 & 105 \\
\hline & (F2) CGATTGAAGTTATTGGGGGAGA & (R2) ACAGATTTTGGAGCAACGGAAT & $\mathrm{F}^{2} \times \mathrm{x}^{+}+$ & 135 \\
\hline
\end{tabular}


The effects of RNA amount and different priming strategies for reverse transcription were also evaluated using cDNA derived from $1 \mu \mathrm{g}$ adult head RNA and tested for $E F 1 \alpha$. The resulting efficiencies were too low for conclusions about the effect of different priming approaches but indicate that increasing the amount of reverse transcribed RNA seems to increase the amount of inhibitor as well. Therefore, a larger RNA volume does not seem to improve the ratio of PCR template and putative inhibitor. To further evaluate whether different amounts of RNA in the cDNA synthesis directly translate into different PCR efficiencies, pooled libraries derived from 500 ng and 190 ng RNA were used to compare the amplification efficiencies of $N A C A$. If all dilution points were included, the cDNA derived from 500 ng RNA was less efficiently amplified whereas under exclusion of the first two dilutions this cDNA was more efficiently amplified. This is in congruence with an inhibitory effect that is directly proportional to the amount of reverse transcribed RNA.

Given the impact of the amplification efficiency on the reproducibility of individual reactions, the amplification efficiencies of the lowly abundant reference gene candidates need to be improved before comparing these genes with other reference gene candidates. From the preliminary results it can be concluded that optimisation of the reverse transcription will not increase the amplification efficiency. This suggests that either the sample preparation procedure needs to be re-evaluated or more extensive primer testing will be required.

\section{Outlook}

The first question to answer will be whether the putative inhibitor equally contaminates all RNA samples or can be linked to a particular sample. Proving whether the inhibitor is present in all samples will require comparing each sample individually to the pool of all samples using the same amplicon. So far, only two larval libraries have been directly compared using two different genes. Since it was shown that PCR inhibitors do not affect all PCR reactions to the same extent [14], this comparison should also be performed on several amplicons. If the inhibitor cannot be associated with a particular sample, the sample preparation itself might need to be optimised. The investigated embryonic libraries were constructed from RNA at concentration between $\sim 60 \mathrm{ng} / \mu \mathrm{L}$ and $\sim 560 \mathrm{ng} / \mu \mathrm{L}$. Collecting more material might increase the concentration of the embryonic RNA samples, allow for 
higher dilutions and therefore expand the range in which lowly abundant genes can be efficiently amplified. Also, for eleven of thirteen reference gene candidates only one primer pair was tested so far. Testing alternative primer pairs might allow for the construction of a set of efficiently amplified candidate genes with different expression levels that can be evaluated for their suitability as internal reference genes [7].

\section{References}

1. Vandesompele J, De Preter K, Pattyn F, Poppe B, Van Roy N, De Paepe A, Speleman F: Accurate normalization of real-time quantitative RT-PCR data by geometric averaging of multiple internal control genes. Gen Biol 2002, 3:research0034.

2. Pfaffl MW: Quantification strategies in real-time PCR. In $A-Z$ of quantitative $P C R$. Edited by Bustin SA. La Jolla, USA: International University Line; 2004:87112.

3. Tang R, Dodd A, Lai D, McNabb W, Love D: Validation of zebrafish (Danio rerio) reference genes for quantitative real-time RT-PCR normalization. Acta Biochim Biophys Sinica 2007, 39:384.

4. Kuijk E, Du Puy L, van Tol H, Haagsman H, Colenbrander B, Roelen B: Validation of reference genes for quantitative RT-PCR studies in porcine oocytes and preimplantation embryos. BMC Dev Biol 2007, 7:58.

5. Sirakov M, Zarrella I, Borra M, Rizzo F, Biffali E, Arnone M, Fiorito G: Selection and validation of a set of reliable reference genes for quantitative RT-PCR studies in the brain of the Cephalopod Mollusc Octopus vulgaris. $B M C \mathrm{Mol}$ Biol 2009, 10:70.

6. Bangaru M, Park F, Hudmon A, Mccallum J, Hogan Q: Quantification of Gene Expression after Painful Nerve Injury: Validation of Optimal Reference Genes. J Mol Neurosci 2012, 46:497-504.

7. Tichopad A, Dzidic A, Pfaffl MW: Improving quantitative real-time RT-PCR reproducibility by boosting primer-linked amplification efficiency. Biotechnol Lett 2002, 24:2053-2056.

8. Rivera A, Weisblat D: And Lophotrochozoa makes three: Notch/Hes signaling in annelid segmentation. Dev Gen Evol 2009, 219:37-43.

9. Kowalewska M, Danska-Bidzinska A, Bakula-Zalewska E, Bidzinski M: 
Identification of suitable reference genes for gene expression measurement in uterine sarcoma and carcinosarcoma tumors. Clin Biochem 2012, 45:368-371.

10. Sarashina I, Yamaguchi H, Haga T, Iijima M, Chiba S, Endo K: Molecular Evolution and Functionally Important Structures of Molluscan Dermatopontin: Implications for the Origins of Molluscan Shell Matrix Proteins. J Mol Evol 2006, 62:307-318.

11. Fabioux C, Corporeau C, Quillien V, Favrel P, Huvet A: In vivo RNA interference in oyster-vasa silencing inhibits germ cell development. FEBS J 2009, 276:25662573.

12. Williams E, Degnan B, Gunter H, Jackson D, Woodcroft BJ, Degnan SM:

Widespread transcriptional changes pre-empt the critical pelagic-benthic transition in the vetigastropod Haliotis asinina. Mol Ecol 2009, 18:1006-1025.

13. Jackson DJ, Wörheide G, Degnan BM: Dynamic expression of ancient and novel molluscan shell genes during ecological transitions. BMC Evol Biol 2007, 7:160.

14. Huggett JF, Novak T, Garson JA, Green C, Morris-Jones SD, Miller RF, Zumla A: Differential susceptibility of PCR reactions to inhibitors: an important and unrecognised phenomenon. BMC Res Notes 2008, 1:70. 
Primer sequences used to isolate Notch pathway orthologues in L. stagnalis.

\begin{tabular}{llll}
\hline $\begin{array}{l}\text { Gene } \\
\text { family }\end{array}$ & $\begin{array}{l}\text { genomic } \\
\text { contig }\end{array}$ & Forward primer (5' to 3') & Reverse primer (5' to 3') \\
\hline DSL & 106533 & TCGACTTTTCTGTGACGGTTACTACTTCA & \\
\hline DSL & 914 & AGACGACCTCCTCGGTCACTACAC & CGATATGACCTGTGACCCCCTGGA \\
\hline DSL & 258 & TGCAAGCCAAGTGGACGTTTTGATTGT & \\
\hline DSL & 27364 & TGTGACACGTATTGTGTTTCAACGAAC & \\
\hline DSL & 85107 & CGCTGTTGGGCGTAGGGAGT & TTCGTGGAGTTCTCTTCTATGCAGGT \\
\hline DSL & 8347 & TCGGCTACTGCGACACTCTGTTCT & ATTAAAACAAATGTGCCTGGAGCAGAG \\
\hline DSL & 198457 & GCCACAACGGCTGGACGGGGGATTACT & GCCCGCCCCAGCCCTCATTACAGTTGC \\
\hline DSL & 221422 & TGTGACCAGAGCAACGGGAGTAAG & \\
\hline DSL & 111341 & TACGGCCAAAATTGCAGTGTGTTC & CTTGGTCACAGACACAGGTATGGAA \\
\hline DSL & 64109 & ACNTTYTTYMGNRTNTG & ARTGNCCRAANTKRTCRTC \\
\hline DSL & 10061 & ATTTTTTTAATCCCAACCTCTCACTTC & TCATCATCTCTGGGTTCACAGAAGTC \\
\hline LNG & 51336 & ATCAGTGACCACACCTGTAAATGTCCT & \\
\hline LNG & 32233 & GAATGGCAACTGCGACAGTGAAT & \\
\hline HES & 1791 & CAGTCTTGGGTTCAATGCCTGTGTCCA & GTGGCGACAACGGTGCGACGAGGGAAG \\
\hline HES & 366599 & TGGAGCTGACTGTCAAATACCTCCGCA & CGTTGGCGCACTCCATGAACCCAGCTT \\
\hline CSL & 165301 & GTNAARATGTTYTAYGGNAA & TACATNCKYTCNGTRTCYTT \\
\hline fringe & 15835 & TGGTTYTGYCAYGTNGAYGAYGA & ATRTANCCCATNGTRCARTCRTC \\
\hline & & & \\
\hline
\end{tabular}

